\title{
Regret in the Newsvendor Model with Demand and Yield Randomness
}

\author{
Zhi Chen \\ Department of Management Sciences, College of Business, City University of Hong Kong, Kowloon Tong, Hong Kong \\ zhi.chen@cityu.edu.hk \\ Weijun Xie \\ Department of Industrial \& Systems Engineering, Virginia Tech, Blacksburg, VA, USA \\ wxie@vt.edu
}

\begin{abstract}
We study the fundamental stochastic newsvendor model that considers both demand and yield randomness. It is usually difficult in practice to describe precisely the joint demand and yield distribution, although partial statistical information and empirical data about this ambiguous distribution are often accessible. We combat the issue of distributional ambiguity by taking a data-driven distributionally robust optimization approach to hedge against all distributions that are sufficiently close to a uniform and discrete distribution of empirical data, where closeness is measured by the type- $\infty$ Wasserstein distance. We adopt the minimax regret decision criterion to assess the optimal order quantity that minimizes the worst-case regret. Several properties about the minimax regret model, including optimality condition, regret bound, and the worst-case distribution, are presented. The optimal order quantity can be determined via an efficient golden section search. We extend the analysis to the Hurwicz criterion model, which generalizes the popular albeit pessimistic maximin model (maximizing the worst-case expected profit) and its (less noticeable) more optimistic counterpart - the maximax model (maximizing the best-case expected profit). Finally, we present numerical comparisons of our data-driven minimax regret model with data-driven models based on the Hurwicz criterion and with a minimax regret model based on partial statistical information on moments.
\end{abstract}

Key words: demand randomness; yield randomness; minimax regret; Hurwicz criterion; type- $\infty$

Wasserstein distance; data-driven decision-making under uncertainty

History: June 23, 2021

\section{Introduction}

The stochastic newsvendor is a foundation for many inventory models, including models of multiitem inventory management, multi-period inventory control, fixed-cost lot sizing, joint inventory and pricing, and supply chain networks. In a stochastic newsvendor model, a firm must determine the order quantity of a single product while facing uncertainty in both supply and demand. In practice, the joint probability distribution of uncertain demand and yield is difficult to elicit precisely. Yet certain types of distributional information - such as support, lower-order moments, and some basic structural properties (e.g., the median, unimodality, and symmetry) — are often available along with 
empirical samples. Hence there exists a collection of distributions that are consistent with the available distributional information; however, the firm is unable to identify the true underlying distribution among them. Of course, if a firm's "optimal" order quantity is based on a misspecified distribution, then it may perform poorly under the true distribution. We mitigate such adverse effects by adopting the distributionally robust optimization (DRO) approach, which seeks an order quantity that performs the best under any conceivable distribution in an ambiguity set of members with common distributional information and whose usefulness has been demonstrated since the origins of inventory management (Scarf 1958).

In the DRO approach, it is essential to encode available distributional information in the ambiguity set, which describes the common properties of all distributions that are robustly hedged. One popular choice is the moment-based ambiguity sets that are mainly concerned with (generalized) moments (see e.g. El Ghaoui et al. 2003, Delage and Ye 2010, Goh and Sim 2010, Wiesemann et al. 2014, Xie and Ahmed 2018, Chen et al. 2019, Li et al. 2019). Since moment estimates are usually obtained from point estimation and so can themselves be uncertain, it follows that moment-based DRO models commit to fixed moments may be susceptible to bias. And even though the availability of further distributional information (e.g., more empirical samples) allows for refining the moment estimates, the moment-based ambiguity sets remain conservative because they do not shrink to a singleton containing only the true distribution. To address these shortcomings, distance-based ambiguity sets are proposed. The distance-based ambiguity sets contain all the distributions that are close to a reference distribution with respect to certain distance measures, among which the most popular are $\phi$-divergences (Ben-Tal et al. 2013, Wang et al. 2016, Jiang and Guan 2018) and the Wasserstein distance (Gao and Kleywegt 2016, Mohajerin Esfahani and Kuhn 2018, Blanchet and Murthy 2019). For the newsvendor model with both demand and yield randomness, we shall adopt a particular distance-based DRO model with a type- $\infty$ Wasserstein ambiguity set, which is constructed directly from empirical data and possesses many attractive features that are detailed in Section 2.2.

A notable advantage of the DRO approach is its ease incorporation into the classical decision criteria, including the Hurwicz and the minimax regret criteria, for decision-making under uncertainty. Successful applications of the DRO approach (chiefly moment-based) partnered with different decision criteria have appeared in the stochastic newsvendor literature. Scarf (1958) and Gallego and Moon (1993) derive the optimal maximin order quantity when only the mean and variance of the random demand are specified, and a substantial amount of efforts has extended applications of the maximin criterion to more complex inventory models (see e.g. Gallego 1998, Gallego et al. 2001, Hanasusanto et al. 2015, Natarajan et al. 2018). Nonetheless, as the maximax criterion typically leads to non-convex optimization problems, DRO newsvendor models incorporating either the maximax criterion or the more general Hurwicz criterion, are relatively scarce: the only work we are 
aware of is Yue et al. (2006) that derive the optimal maximax order quantity when only the mean and variance of the random demand are known. By definition, the maximin (maximax) criterion is conservative (aggressive) because it focuses on the worst-case (best-case) circumstance. To avoid such extremes, the minimax regret criterion is investigated in the newsvendor models by Vairaktarakis (2000), Yue et al. (2006), Perakis and Roels (2008), and Zhu et al. (2013), and is adopted by Lin and Ng (2011) to select from independent markets for a single product. In particular, Perakis and Roels (2008) analyze various moment-based ambiguity sets and reveal that the minimax regret criterion is typically much less conservative than the maximin criterion. Motivated by its tractability and potential to mitigate aggressive or conservative extreme, we focus on the minimax regret criterion for our interested newsvendor model with demand and yield randomness.

In most of the cited DRO newsvendor models, demand is the only source of randomness, and thus less attention has been paid to the yield randomness stemming from the supply side. Many factors - such as weather conditions, disasters, and unreliable procurement as well as uncertainties in production and transportation processes - could be responsible for the cause of yield randomness. We refer to Yano and Lee (1995) for a comprehensive survey on inventory management with random yield and to Inderfurth and Kiesmüller (2015) for more recent algorithmic developments. Yield randomness is also related to the research on supply disruption (see e.g. Tomlin and Wang 2011, DeCroix 2013, Tang et al. 2014) and it has witnessed wide-spread applications in supply chain outsourcing (e.g. Kouvelis and Li 2013, Tan et al. 2016), competition (Tang and Kouvelis 2011, Gupta et al. 2015), and coordination (Tang and Kouvelis 2014, Bimpikis et al. 2018).

In this paper, we study a data-driven and distributionally robust newsvendor model with both demand and yield randomness. Here the optimal order quantity is assessed in terms of the minimax regret criterion and the Hurwicz criterion. Our key contributions may be summarized as follows.

1. We derive several notable properties - including optimality condition, regret bound, and worstcase distribution - of our data-driven minimax regret newsvendor model with both demand and yield randomness. Leveraging these properties, we propose an efficient golden section search method for obtaining the optimal minimax regret order quantity. Our results for the minimax regret newsvendor model can be strengthened when only demand is random.

2. We study the data-driven newsvendor model under the Hurwicz criterion, where the optimal order quantity can be obtained by solving a scalable linear program reformulation or comparing the objective values among a small number of known candidates. All results about the Hurwicz criterion model can be applied in a straightforward manner to its special cases of the pessimistic maximin model and the optimistic maximax model.

3. Our data-driven approach complements existing literature on moment-based minimax regret newsvendor models. It also contributes to the literature on stochastic newsvendor models under 
distributional ambiguity, where less attention has been paid to the Hurwicz criterion model and yield randomness.

4. Our model, which considers the extreme-case expected performance from the distributionally robust perspective, readily covers the traditional robust perspective that focuses on the performance under an extreme scenario. From the traditional robust perspective, optimal order quantities in the minimax regret model and in the Hurwicz criterion model each have a closed form. Hence we can compare optimal order quantities under different criteria when the source of randomness is the demand side, the supply side, or both sides.

5. Our minimax regret model based on the type- $\infty$ Wasserstein distance shows encouraging outof-sample performances in data-driven numerical experiments. Compared with the maximax and maximin decision criteria, the minimax regret approach can perform much better and can be less sensitive to the training sample size and profit margin. Besides, we show that our distance-based minimax regret model, which is purely data-driven, performs competitively against the moment-based one that requires proper specifications of the ambiguity set.

Notations. By $[N] \triangleq\{1,2, \ldots, N\}$ we denote the set of positive running indices up to $N$. We use $\tilde{\boldsymbol{w}} \sim \mathbb{P}$ to signify a (possibly multi-dimensional) random variable $\tilde{\boldsymbol{w}}$ distributed as $\mathbb{P}$. The symbol $\delta_{\hat{\boldsymbol{w}}}$ stands for the Dirac distribution that places unit mass on the realization $\hat{\boldsymbol{w}}$. We adopt the convention $a / 0=+\infty$ for any $a>0$.

\section{Model Formulation, Ambiguity Set, and Decision Criteria}

In this section, we present the mathematical formulation of the stochastic newsvendor model with uncertain demand and yield. Also, we review different decision criteria for ambiguous joint demand and yield distribution before introducing the data-driven type- $\infty$ Wasserstein ambiguity set.

\subsection{Model Formulation}

We consider a stochastic newsvendor model in which a firm needs to determine the optimal order quantity of a product when facing uncertainty in random supply $\tilde{u}$ and in random demand $\tilde{v}$. The product's unit price and cost are $p$ and $c$, respectively. If a product is profitable then $p>c>0$. Given an order quantity $x$, the profit function under a yield and demand realization $(u, v)$ is

$$
\Pi(x, u, v)=(p-c) u x-p(u x-v)^{+} .
$$

Here we model random yield by adopting a widely used approach, stochastically proportional yield, which specifies a yield rate $\tilde{u}$ that lies between zero and one and is independent of the order quantity (see Yano and Lee 1995). 
If the random yield $\tilde{u}$ and the random demand $\tilde{v}$ together follows a known joint distribution $(\tilde{u}, \tilde{v}) \sim \mathbb{P}_{\mathrm{o}}$ (not necessarily independent), then a risk-neutral firm's optimal order quantity $x_{\mathrm{o}}^{\star}$, which achieves the maximal expected profit of the following stochastic problem

$$
\max _{x \geq 0} \mathbb{E}_{\mathbb{P}_{\mathrm{o}}}[\Pi(x, \tilde{u}, \tilde{v})]
$$

can be characterized by the following optimality condition (Käki et al. 2015):

$$
x_{\mathrm{o}}^{\star}=\inf \left\{x: \frac{\mathbb{E}_{\mathbb{P}_{\mathrm{o}}}\left[\tilde{u} \cdot \mathbb{I}_{\{\tilde{v} \leq \tilde{u} x\}}\right]}{\mathbb{E}_{\mathbb{P}_{\mathrm{o}}}[\tilde{u}]} \geq \frac{p-c}{p}\right\} .
$$

\subsection{Type- $\infty$ Wasserstein Ball}

We employ an ambiguity set in the form of a ball in the space of probability distributions with respect to the type- $\infty$ Wasserstein distance. The type- $\infty$ Wasserstein distance between two distributions, $\mathbb{P}_{1}$ and $\mathbb{P}_{2}$, is defined mathematically as

$$
d_{\infty}\left(\mathbb{P}_{1}, \mathbb{P}_{2}\right) \triangleq \inf _{\mathbb{Q} \in \mathcal{P}\left(\mathbb{P}_{1}, \mathbb{P}_{2}, \mathcal{W}\right)} \mathbb{Q} \text {-ess sup }\left\|\tilde{\boldsymbol{w}}_{1}-\tilde{\boldsymbol{w}}_{2}\right\|
$$

where $\tilde{\boldsymbol{w}}_{1} \sim \mathbb{P}_{1}$ and $\tilde{\boldsymbol{w}}_{2} \sim \mathbb{P}_{2}$ and where $\mathcal{P}\left(\mathbb{P}_{1}, \mathbb{P}_{2}, \mathcal{W}\right)$ represents the set of all distributions on the support $\mathcal{W} \times \mathcal{W}$ with marginals $\mathbb{P}_{1}$ and $\mathbb{P}_{2}$. The notation $\mathbb{Q}$-ess sup $\|\cdot\|$ expresses the essential supremum of the norm $\|\cdot\|$ with respect to the joint distribution $\mathbb{Q}$, which is formally defined as

$$
\mathbb{Q}-\operatorname{ess} \sup \left\|\tilde{\boldsymbol{w}}_{1}-\tilde{\boldsymbol{w}}_{2}\right\| \triangleq \inf \left\{\Theta: \mathbb{Q}\left[\left\|\tilde{\boldsymbol{w}}_{1}-\tilde{\boldsymbol{w}}_{2}\right\|>\Theta\right]=0\right\}
$$

The type- $\infty$ Wasserstein ambiguity set is then defined as a ball — of radius $\theta \geq 0$ with respect to the type- $\infty$ Wasserstein distance - that is centered at a discrete empirical distribution $\hat{\mathbb{P}}$ :

$$
\mathcal{F}_{\infty}(\theta) \triangleq\left\{\mathbb{P} \in \mathcal{P}(\mathcal{W}) \mid d_{\infty}(\mathbb{P}, \hat{\mathbb{P}}) \leq \theta\right\}
$$

here $\mathcal{P}(\mathcal{W})$ represents the set of all distributions on the support set $\mathcal{W}$, and the empirical distribution $\hat{\mathbb{P}} \triangleq \frac{1}{N} \sum_{i \in[N]} \delta_{\hat{\boldsymbol{w}}_{i}}$ is uniformly distributed on $N$ empirical samples $\hat{\boldsymbol{w}}_{1}, \ldots, \hat{\boldsymbol{w}}_{N}$ of the random variable $\tilde{\boldsymbol{w}}$. Bertsimas et al. (2018) show that a distribution $\mathbb{P}$ in the type- $\infty$ Wasserstein ball is essentially a mixture distribution $\mathbb{P}=\frac{1}{N} \sum_{i \in[N]} \mathbb{P}_{i}$ consisting of ambiguous components such that, for every $i \in[N]$, the only information about the component $\mathbb{P}_{i}$ is its support:

$$
\mathbb{P}_{i} \in \mathcal{F}_{i}(\theta)=\left\{\mathbb{P} \in \mathcal{P}(\mathcal{W}) \mid \mathbb{P}\left[\left\|\tilde{\boldsymbol{w}}-\hat{\boldsymbol{w}}_{i}\right\| \leq \theta\right]=1\right\}
$$

That is to say, the type- $\infty$ Wasserstein ball can be represented as

$$
\mathcal{F}_{\infty}(\theta)=\left\{\mathbb{P} \in \mathcal{P}(\mathcal{W}) \mid \mathbb{P}=\frac{1}{N} \sum_{i \in[N]} \mathbb{P}_{i}, \mathbb{P}_{i} \in \mathcal{F}_{i}(\theta) \forall i \in[N]\right\}
$$


Note that since the reference distribution is constructed from finite empirical samples, it is likely that the true distribution has a larger support than those observed empirically. Then $\phi$-divergences might not be able to measure the distance between the empirical distribution and true one effectively (section 1.1 in Gao and Kleywegt 2016). Thus, we adopt the more appropriate Wasserstein distance.

Two notable cases of type- $\infty$ Wasserstein ball deserve particular attentions. In the first special case, where the Wasserstein radius is zero, the type- $\infty$ Wasserstein ball shrinks to a singleton set containing only the empirical distribution: $\mathcal{F}_{\infty}(0)=\{\hat{\mathbb{P}}\}=\left\{\frac{1}{N} \sum_{i \in[N]} \delta_{\hat{\boldsymbol{w}}_{i}}\right\}$. In this case, the worst-case expectation becomes the expectation (or sample average) with respect to the empirical distribution. In other words, for any function $g: \mathcal{W} \mapsto \mathbb{R}$ we have

$$
\max _{\mathbb{P}_{\mathcal{F}} \mathcal{F}_{\infty}(0)} \mathbb{E}_{\mathbb{P}}[g(\tilde{\boldsymbol{w}})]=\mathbb{E}_{\hat{\mathbb{P}}}[g(\tilde{\boldsymbol{w}})]=\frac{1}{N} \sum_{i \in[N]} g\left(\hat{w}_{i}\right) .
$$

When $N$ increases, the empirical distribution $\hat{\mathbb{P}}$ is expected to more nearly approximate the true distribution $\mathbb{P}_{\mathrm{o}}$ and thus to set a smaller Wasserstein radius $\theta$ (i.e., since the center becomes more trustworthy). Under a mild assumption of compact support, Trillos and Slepčev (2015) show that the type- $\infty$ Wasserstein ball converges to the true distribution $\mathbb{P}_{\mathrm{o}}$ as $N$ approaches infinity.

In the second special case, for which there are $N=1$ empirical samples, the type- $\infty$ Wasserstein ball becomes $\mathcal{F}_{\infty}(\theta)=\{\mathbb{P} \in \mathcal{P}(\mathcal{W}) \mid \mathbb{P}[\|\tilde{\boldsymbol{w}}-\hat{\boldsymbol{w}}\| \leq \theta]=1\}$ and degenerates to an uncertainty set $\hat{\mathcal{W}}=\{\boldsymbol{w} \in \mathcal{W} \mid\|\boldsymbol{w}-\hat{\boldsymbol{w}}\| \leq \theta\}$ for all possible realizations of the random variable $\tilde{\boldsymbol{w}}$. In this case, the worst-case expectation in the data-driven distributionally robust optimization model recovers the traditional robust optimization model because for any function $g: \hat{\mathcal{W}} \mapsto \mathbb{R}$ it holds that:

$$
\max _{\mathbb{P} \in \mathcal{F}_{\infty}(\theta)} \mathbb{E}_{\mathbb{P}}[g(\tilde{\boldsymbol{w}})]=\max _{\boldsymbol{w} \in \hat{\mathcal{W}}} g(\boldsymbol{w})
$$

Owing to its extremely modest requirement for distributional information, the traditional robust perspective is usually more computationally attractive while still being able to hedge against uncertainty; see Ben-Tal and Nemirovski (2000) and Bertsimas and Sim (2004).

Many scholars contributing to the DRO literature study type- $\rho$ Wasserstein balls of the form

$$
\mathcal{F}_{\rho}(\theta) \triangleq\left\{\mathbb{P} \in \mathcal{P}(\mathcal{W}) \mid d_{\rho}(\mathbb{P}, \hat{\mathbb{P}}) \leq \theta\right\}
$$

here, for any finite $\rho \in[1, \infty)$, the type- $\rho$ Wasserstein distance is defined as

$$
d_{\rho}\left(\mathbb{P}_{1}, \mathbb{P}_{2}\right) \triangleq \inf _{\mathbb{Q} \in \mathcal{P}\left(\mathbb{P}_{1}, \mathbb{P}_{2}, \mathcal{W}\right)} \sqrt[\rho]{\mathbb{E}_{\mathbb{Q}}\left[\left\|\tilde{\boldsymbol{w}}_{1}-\tilde{\boldsymbol{w}}_{2}\right\|^{\rho}\right]}
$$

It is intuitive that the type- $\rho$ Wasserstein distance converges to the type- $\infty$ Wasserstein distance as $\rho$ approaches infinity (Givens and Shortt 1984). Different types of Wasserstein ball share quite similar statistical properties, such as finite sample guarantees and convergence (Xie et al. 2021), and 
they can be nicely represented in the unified format of an ambiguity set (Chen et al. 2020). However, depending on the problem context, various ball types can lead to models with different levels of tractability (see Xie 2020). For our minimax regret newsvendor model introduced subsequently, we focus on the type- $\infty$ Wasserstein ball as it leads to a reformulation whose optimal order quantity can be obtained through a tractable golden section search.

\subsection{Decision Criteria}

The exact joint demand and yield distribution is seldom known with much precision, so we are motivated to consider the more appealing DRO approach in which the firm does not commit to a single distribution and instead seeks an order quantity that performs well under any conceivable distribution in an ambiguity set $\mathcal{F}$. Another advantage of the DRO approach is that it can be seamlessly integrated with several classical decision criteria for making decisions under uncertainty.

Minimax regret criterion. The notion of regret was formalized first by Savage (1951) and is known also as the expected value of distributional information. Given an order quantity $x$ and a joint demand and yield distribution $\mathbb{P}$, regret is measured as the difference in profits between $x$ and the optimal order quantity $y$ under the distribution $\mathbb{P}$ :

$$
\max _{y}\left\{\mathbb{E}_{\mathbb{P}}[\Pi(y, \tilde{u}, \tilde{v})]\right\}-\mathbb{E}_{\mathbb{P}}[\Pi(x, \tilde{u}, \tilde{v})]=\max _{y}\left\{\mathbb{E}_{\mathbb{P}}[\Pi(y, \tilde{u}, \tilde{v})-\Pi(x, \tilde{u}, \tilde{v})]\right\} .
$$

The worst-case regret resulting from distributional ambiguity amounts to the largest possible regret across all possible distributions within the ambiguity set $\mathcal{F}$; that is,

$$
\gamma(x)=\max _{\mathbb{P} \in \mathcal{F}} \max _{y}\left\{\mathbb{E}_{\mathbb{P}}[\Pi(y, \tilde{u}, \tilde{v})-\Pi(x, \tilde{u}, \tilde{v})]\right\} .
$$

The minimax regret criterion, which seeks the least worst-case regret, solves

$$
\gamma^{\star}=\min _{x} \max _{\mathbb{P} \in \mathcal{F}} \max _{y}\left\{\mathbb{E}_{\mathbb{P}}[\Pi(y, \tilde{u}, \tilde{v})-\Pi(x, \tilde{u}, \tilde{v})]\right\} .
$$

We refer to Lim et al. (2006), Kouvelis and Yu (2013), Bertsimas and Dunning (2020), and Poursoltani and Delage (2019) for general moment-based robust problems under minimax regret.

Hurwicz criterion. The Hurwicz criterion articulates a critical tradeoff between pessimism and optimism in decision-making under uncertainty (Hurwicz 1951, Luce and Raiffa 1989). Given a level of optimism $\lambda \in[0,1]$, the Hurwicz criterion newsvendor model solves

$$
\max _{x}\left\{\lambda \cdot \max _{\mathbb{P} \in \mathcal{F}} \mathbb{E}_{\mathbb{P}}[\Pi(x, \tilde{u}, \tilde{v})]+(1-\lambda) \cdot \min _{\mathbb{P} \in \mathcal{F}} \mathbb{E}_{\mathbb{P}}[\Pi(x, \tilde{u}, \tilde{v})]\right\},
$$

which aims to find an optimal order quantity attaining a maximal weighted average of the best- and worst-case expected profits in the ambiguity set $\mathcal{F}$ of all conceivable distributions. On the one hand, if $\lambda=1$ then the Hurwicz criterion recovers the most optimistic maximax criterion that solves

$$
\max _{x} \max _{\mathbb{P} \in \mathcal{F}} \mathbb{E}_{\mathbb{P}}[\Pi(x, \tilde{u}, \tilde{v})],
$$


whose optimal order quantity aggressively maximizes its best-case expected profit. On the other hand, if $\lambda=0$ then the Hurwicz criterion recovers the most pessimistic maximin criterion that solves

$$
\max _{x} \min _{\mathbb{P} \in \mathcal{F}} \mathbb{E}_{\mathbb{P}}[\Pi(x, \tilde{u}, \tilde{v})]
$$

whose optimal order quantity conservatively maximizes its worst-case expected profit. The Hurwicz criterion, including its special cases of maximax and maximin criteria, enjoys strong economical and empirical justifications in decision theory (Ellsberg 1961, Gilboa and Schmeidler 1989). However, it may not be easy for decision-makers to truthfully articulate the level of optimism.

The maximax (resp., maximin) criterion provides the most optimistic (resp., pessimistic) perception on the expected performance when the distribution arises from the ambiguity set $\mathcal{F}$ and the following difference between the maximax and the maximin profits,

$$
\Gamma^{\star}=\max _{x} \max _{\mathbb{P} \in \mathcal{F}} \mathbb{E}_{\mathbb{P}}[\Pi(x, \tilde{u}, \tilde{v})]-\max _{x} \min _{\mathbb{P} \in \mathcal{F}} \mathbb{E}_{\mathbb{P}}[\Pi(x, \tilde{u}, \tilde{v})],
$$

measures the level of uncertainty (i.e., the range of possible profits) of the newsvendor model.

Comparing $\gamma^{\star}$ with $\Gamma^{\star}$, the ratio $\gamma^{\star} / \Gamma^{\star}$ quantifies the percentage reduction in profit uncertainty due to the minimax regret criterion. For any fixed $x$ it holds that

$$
\max _{\mathbb{P} \in \mathcal{F}} \max _{y}\left\{\mathbb{E}_{\mathbb{P}}[\Pi(y, \tilde{u}, \tilde{v})-\Pi(x, \tilde{u}, \tilde{v})]\right\} \leq \max _{\mathbb{P} \in \mathcal{F}}\left\{\max _{y} \mathbb{E}_{\mathbb{P}}[\Pi(y, \tilde{u}, \tilde{v})]\right\}-\min _{\mathbb{P} \in \mathcal{F}} \mathbb{E}_{\mathbb{P}}[\Pi(x, \tilde{u}, \tilde{v})]
$$

implying the following inequalities:

$$
\begin{aligned}
\min _{x} \max _{\mathbb{P} \in \mathcal{F}} \max _{y}\left\{\mathbb{E}_{\mathbb{P}}[\Pi(y, \tilde{u}, \tilde{v})-\Pi(x, \tilde{u}, \tilde{v})]\right\} & \leq \min _{x}\left\{\max _{\mathbb{P} \in \mathcal{F}} \max _{y} \mathbb{E}_{\mathbb{P}}[\Pi(y, \tilde{u}, \tilde{v})]-\min _{\mathbb{P} \in \mathcal{F}} \mathbb{E}_{\mathbb{P}}[\Pi(x, \tilde{u}, \tilde{v})]\right\} \\
& =\max _{x} \max _{\mathbb{P} \in \mathcal{F}} \mathbb{E}_{\mathbb{P}}[\Pi(x, \tilde{u}, \tilde{v})]-\max _{x} \min _{\mathbb{P} \in \mathcal{F}} \mathbb{E}_{\mathbb{P}}[\Pi(x, \tilde{u}, \tilde{v})] .
\end{aligned}
$$

Hence the ratio $\gamma^{\star} / \Gamma^{\star}$ cannot exceed unity ${ }^{1}$. Indeed, it is often much less than one, as supported by the numerical evidence in Perakis and Roels (2008) for moment-based models with only demand randomness and by our subsequent numerical experiments for distance-based models with both demand and yield randomness. This shows an appealing feature of the minimax regret decision criterion to quantify the profit uncertainty more tightly, avoiding it from being aggressive or conservative. Therefore, in this paper we will mainly focus on the desirable minimax regret criterion.

\section{Minimax Regret Model with Both Demand and Yield Randomness}

The minimax regret newsvendor model with the type- $\infty$ Wasserstein ball of radius $\theta>0$ solves

$$
\min _{x} \max _{\mathbb{P} \in \mathcal{F}_{\infty}(\theta)} \max _{y}\left\{\mathbb{E}_{\mathbb{P}}[\Pi(y, \tilde{u}, \tilde{v})-\Pi(x, \tilde{u}, \tilde{v})]\right\}
$$

${ }^{1}$ We define $\frac{0}{0}=1$ for the extreme cases, e.g., when $\mathcal{F}$ is a singleton set. 
Thus the firm seeks an optimal order quantity that attains the least worst-case regret across all distributions in close proximity to the empirical distribution $\hat{\mathbb{P}} \triangleq \frac{1}{N} \sum_{i \in[N]} \delta_{\left(\hat{u}_{i}, \hat{v}_{i}\right)}$. Here $\left\{\left(\hat{u}_{i}, \hat{v}_{i}\right)\right\}_{i \in[N]}$ are empirical samples of yield and demand realization, and we assume that support sets of yield and demand are $[\underline{u}, \bar{u}] \subseteq[0,1]$ and $[\underline{v}, \bar{v}] \subseteq \mathbb{R}_{+}$, respectively. Observe that we can switch the order of maximization problems and obtain

$$
\min _{x} \max _{y} \max _{\mathbb{P} \in \mathcal{F}_{\infty}(\theta)}\left\{\mathbb{E}_{\mathbb{P}}[\Pi(y, \tilde{u}, \tilde{v})-\Pi(x, \tilde{u}, \tilde{v})]\right\},
$$

which-given that any distribution $\mathbb{P} \in \mathcal{F}_{\infty}(\theta)$ can be re-expressed as a mixture distribution of ambiguous components $\mathbb{P}_{i}, i \in[N]$-is equivalent to

$$
\min _{x} \max _{y} \frac{1}{N} \sum_{i \in[N]} \max _{\mathbb{P}_{i} \in \mathcal{F}_{i}(\theta)}\left\{\mathbb{E}_{\mathbb{P}_{i}}[\Pi(y, \tilde{u}, \tilde{v})-\Pi(x, \tilde{u}, \tilde{v})]\right\} .
$$

For each of these ambiguous mixture components $\mathbb{P}_{i}, i \in[N]$, only the support information is known. Specifically, we assume only marginal supports of yield and demand are known: for any $\mathbb{P}_{i} \in \mathcal{F}_{i}(\theta)$ we have $\mathbb{P}_{i}\left[\left(\tilde{u}_{i}, \tilde{v}_{i}\right) \in \mathcal{U}_{i} \times \mathcal{V}_{i}\right]=1$. The marginal uncertainty sets for yield and demand are

$$
\mathcal{U}_{i}=\left[\hat{u}_{i}-\theta / \kappa, \hat{u}_{i}+\theta / \kappa\right] \cap[\underline{u}, \bar{u}] \triangleq\left[\underline{u}_{i}, \bar{u}_{i}\right] \quad \text { and } \quad \mathcal{V}_{i}=\left[\hat{v}_{i}-\theta, \hat{v}_{i}+\theta\right] \cap[\underline{v}, \bar{v}] \triangleq\left[\underline{v}_{i}, \bar{v}_{i}\right],
$$

where $\kappa>0$ is a scaling factor to normalize the yield and demand of similar magnitude. The specification in (4) can be obtained by choosing, in the definition of type- $\infty$ Wasserstein distance, a scaled $L_{\infty}$-norm in $\mathbb{R}^{2}$ as $\|(u, v)\|_{\infty}=\max \{\kappa|u|,|v|\}$.

With the specification of support information, we can now rewrite problem (3) as

$$
\min _{x} \max _{y} \frac{1}{N} \sum_{i \in[N]} \max _{(u, v) \in \mathcal{U}_{i} \times \mathcal{V}_{i}} \Lambda(x, y, u, v)
$$

here $\Lambda(x, y, u, v) \triangleq \Pi(y, u, v)-\Pi(x, u, v)=(p-c)(y-x) u+p(u x-v)^{+}-p(u y-v)^{+}$. Since the function $\Lambda(x, y, u, v)$ is convex in $x$ for any fixed values of $y, u$, and $v$, the objective function of the outer minimization problem is indeed convex in the order quantity $x$ (Boyd and Vandenberghe 2004, section 3). Therefore, problem (5) - that is, the minimax regret newsvendor model - is a onedimensional convex program, whose optimal solution can be found using the golden section search that we next develop in detail.

\subsection{Properties of Minimax Regret Model}

The key ingredient in solving a convex formulation of the minimax regret newsvendor model (5) is efficiently evaluating the worst-case regret associated with a fixed order quantity $x$, which is

$$
\max _{y} \frac{1}{N} \sum_{i \in[N]} \max _{u \in \mathcal{U}_{i}} \max _{v \in \mathcal{V}_{i}} \Lambda(x, y, u, v) .
$$


Indeed, the worst-case regret can be re-expressed as the maximum of two separate components,

$$
\max \left\{\max _{y \geq x} \frac{1}{N} \sum_{i \in[N]} \max _{u \in \mathcal{U}_{i}} \max _{v \in \mathcal{V}_{i}} \Lambda(x, y, u, v), \max _{y \leq x} \frac{1}{N} \sum_{i \in[N]} \max _{u \in \mathcal{U}_{i}} \max _{v \in \mathcal{V}_{i}} \Lambda(x, y, u, v)\right\},
$$

where the first (resp., second) component corresponds to the case where the auxiliary decision $y$ is not smaller (resp., not larger) than the order quantity $x$. The technique of splitting cases into $y \geq x$ and $y \leq x$ is similar to proposition 1 in Perakis and Roels (2008), where the authors study a minimax regret model with only demand randomness under moment-based ambiguity sets. In particular, Perakis and Roels (2008) formulate the problem of identifying the worst-case demand scenario as a moment problem (Popescu 2005) and analyze its dual as a semi-infinite linear optimization problem that is quite involved. We anticipate that the dual would becomes much harder to analyze with both demand and yield randomness. Our derivation is significantly different: we explore the representation of member distributions in the type- $\infty$ Wasserstein ball as an ambiguous mixture distribution so that we are able to identify the worst-case demand and yield scenarios separately yet efficiently. We start by showing that for any realization of random yield, the worst-case demand scenario depends only on the relationship between $x$ and $y$.

Lemma 1. For any $u \in[0,1]$ and any interval $\mathcal{V}=[\underline{v}, \bar{v}]$, the following result holds:

$$
\max _{v \in \mathcal{V}} \Lambda(x, y, u, v)= \begin{cases}(p-c)(y-x) u+p(u x-\bar{v})^{+}-p(u y-\bar{v})^{+} & \text {if } y \geq x \\ (p-c)(y-x) u+p(u x-\underline{v})^{+}-p(u y-\underline{v})^{+} & \text {if } y \leq x\end{cases}
$$

Since in Lemma 1, the realization of random yield is fixed, we obtain the corresponding worst-case demand scenarios of $y \geq x$ and $y \leq x$ for the minimax regret model (with only demand randomness) of Perakis and Roels (2008); see theorem 1 therein. We can now use Lemma 1 to express the worst-case regret of an order quantity $x$ as

$$
\max \left\{\max _{y \geq x} \frac{1}{N} \sum_{i \in[N]} \max _{u \in \mathcal{U}_{i}} \Lambda\left(x, y, u, \bar{v}_{i}\right), \max _{y \leq x} \frac{1}{N} \sum_{i \in[N]} \max _{u \in \mathcal{U}_{i}} \Lambda\left(x, y, u, \underline{v}_{i}\right)\right\} .
$$

Next we discuss the worst-case scenario of random yield, which is more difficult to find than that of random demand - a difference that reveals the more complex effects of random yield on the minimax regret newsvendor model. Our main task is to solve an optimization problem $\max _{u \in \mathcal{U}} \Lambda(x, y, u, v)$ over the interval $\mathcal{U}=[\underline{u}, \bar{u}]$, given a fixed value of $v$. We treat the two cases, $y \geq x$ and $y \leq x$, separately, and we summarize the Lemma 2 results in Table 1.

Lemma 2. The optimal objective value $\Lambda^{\star}$ and the optimal solution $u^{\star}$ to the optimization problem $\max _{u \in[\underline{u}, \bar{u}]} \Lambda(x, y, u, v)$ are summarized in Table 1. 


\begin{tabular}{|c|c|c|c|c|c|}
\hline Condition & $\Lambda^{\star}$ & $u^{\star}$ & Condition & $\Lambda^{\star}$ & $u^{\star}$ \\
\hline$v / \underline{u} \geq v / \bar{u} \geq y \geq x$ & $(p-c) \bar{u} y-(p-c) \bar{u} x$ & $\bar{u}$ & $v / \underline{u} \geq v / \bar{u} \geq x \geq y$ & $(p-c) \underline{u} y-(p-c) \underline{u} x$ & $\underline{u}$ \\
\hline$v / \underline{u} \geq y \geq v / \bar{u} \geq x$ & $-(p-c) \frac{x v}{y}+(p-c) v$ & $\frac{v}{y}$ & $v / \underline{u} \geq x \geq v / \bar{u} \geq y$ & $\max \left\{\Lambda_{1}, \Lambda_{2}\right\}^{\mathrm{I}}$ & $\underline{u}(\bar{u})^{\mathrm{II}}$ \\
\hline$v / \underline{u} \geq y \geq x \geq v / \bar{u}$ & $-(p-c) \frac{x v}{y}+(p-c) v$ & $\frac{v}{y}$ & $v / \underline{u} \geq x \geq y \geq v / \bar{u}$ & $-c \bar{u} y+c \bar{u} x$ & $\bar{u}$ \\
\hline$y \geq v / \underline{u} \geq v / \bar{u} \geq x$ & $-c \underline{u} y-(p-c) \underline{u} x+p v$ & $\underline{u}$ & $x \geq v / \underline{u} \geq v / \bar{u} \geq y$ & $(p-c) \bar{u} y+c \bar{u} x-p v$ & $\bar{u}$ \\
\hline$y \geq v / \underline{u} \geq x \geq v / \bar{u}$ & $-c \underline{u} y-(p-c) \underline{u} x+p v$ & $\underline{u}$ & $x \geq v / \underline{u} \geq y \geq v / \bar{u}$ & $-c \bar{u} y+c \bar{u} x$ & $\bar{u}$ \\
\hline$y \geq x \geq v / \underline{u} \geq v / \bar{u}$ & $-c \underline{u} y+c \underline{u} x$ & $\underline{u}$ & $x \geq y \geq v / \underline{u} \geq v / \bar{u}$ & $-c \bar{u} y+c \bar{u} x$ & $\bar{u}$ \\
\hline
\end{tabular}

Table 1 Optimal objective value and optimal solution for the cases of $y \geq x$ (left) and $y \leq x$ (right).

Using the results in Lemmas 1 and 2, we can determine an interval in which the optimal minimax regret order quantity lies. Determining that interval would be helpful for initializing the golden section searching space, with running time complexity in a logarithmic order of the length of that interval, whose boundary points has closed-form expressions according to the following result.

THEOREM 1. The optimal order quantity $x_{\mathrm{R}}^{\star}$ in the minimax regret newsvendor model (5) lies in the interval $[\underline{x}, \bar{x}]$, where

$$
\underline{x}=\min _{i \in[N]}\left\{\frac{\underline{v}_{i}}{\bar{u}_{i}}\right\} \quad \text { and } \quad \bar{x}=\max \left\{\max _{i \in \mathcal{N}}\left\{\frac{\bar{v}_{i}}{\underline{u}_{i}}\right\}, \frac{(p-c) \sum_{i \in[N] \backslash \mathcal{N}} \bar{v}_{i}}{c \sum_{i \in \mathcal{N}} \underline{u}_{i}}\right\} ;
$$

in the latter equality, $\mathcal{N}=\left\{i \in[N]: \underline{u}_{i}>0\right\}$ and $\sum_{i \in[N] \backslash \mathcal{N}} \bar{v}_{i}=0$ if $\mathcal{N}=[N]$. Moreover, the minimax regret newsvendor model (5) is equivalent to

$$
\min _{\underline{x} \leq x \leq \bar{x}} \max \left\{\max _{x \leq y \leq \bar{x}} \frac{1}{N} \sum_{i \in[N]} \max _{u \in \mathcal{U}_{i}} \Lambda\left(x, y, u, \bar{v}_{i}\right), \max _{\underline{x} \leq y \leq x} \frac{1}{N} \sum_{i \in[N]} \max _{u \in \mathcal{U}_{i}} \Lambda\left(x, y, u, \underline{v}_{i}\right)\right\} .
$$

It is worth noting that, for any optimal minimax regret order quantity $x_{\mathrm{R}}^{\star}$, the two components within the first maximum of problem (6) should be equal.

Proposition 1. The optimal order quantity $x_{\mathrm{R}}^{\star}$ in the minimax regret newsvendor model (6) satisfies the following optimality condition:

$$
\max _{x_{\mathrm{R}}^{\star} \leq y \leq \bar{x}} \sum_{i \in[N]} \max _{u \in \mathcal{U}_{i}} \Lambda\left(x_{\mathrm{R}}^{\star}, y, u, \bar{v}_{i}\right)=\max _{\underline{x} \leq y \leq x_{\mathrm{R}}^{\star}} \sum_{i \in[N]} \max _{u \in \mathcal{U}_{i}} \Lambda\left(x_{\mathrm{R}}^{\star}, y, u, \underline{v}_{i}\right) .
$$

The optimal condition states that "the quantity that minimizes the maximal regret is such that the regret of ordering too little is equal to the regret of ordering too much" (Perakis and Roels 2008, 
p. 191). This remarkable property was first identified by Perakis and Roels (2008, proposition 1) for the minimax regret newsvendor model with only demand randomness under any nonempty convex ambiguity set. Proposition 1 shows that such a property also holds for the minimax regret newsvendor model with both demand and yield randomness and with a type- $\infty$ Wasserstein ball.

The optimal interval $[\underline{x}, \bar{x}]$ in reformulation (6) strengthens the "naïve" bound

$$
[\underline{x}, \bar{x}] \subseteq\left[\frac{\min _{i \in[N]}\left\{\underline{v}_{i}\right\}}{\max _{i \in[N]}\left\{\bar{u}_{i}\right\}}, \frac{\max _{i \in[N]}\left\{\bar{v}_{i}\right\}}{\min _{i \in[N]}\left\{\underline{u}_{i}\right\}}\right],
$$

which is based solely on a rough estimation derived from dividing the smallest (resp., largest) demand $\min _{i \in[N]}\left\{\underline{v}_{i}\right\}$ (resp., $\max _{i \in[N]}\left\{\bar{v}_{i}\right\}$ ) by the corresponding best (resp., worst) yield. We next derive an upper bound on the worst-case regret associated with the optimal minimax regret order quantity $x_{\mathrm{R}}^{\star}$. Our proof explores the more precisely identified optimal interval in which $x_{\mathrm{R}}^{\star}$ lies.

Proposition 2. In the minimax regret newsvendor model (6), the optimal (i.e., the least) worstcase regret of the optimal order quantity $x_{\mathrm{R}}^{\star}$ is bounded from above by $2(\bar{x}-\underline{x}) \max \{p-c, c\} \theta$, which tends to zero as the Wasserstein radius $\theta$ shrinks to zero when the sample size $N$ approaches infinity.

REMARK 1. When the random demand and yield has a compact support and a probability density/mass function bounded from below by a positive constant almost everywhere, theorem 1.1 in Trillos and Slepčev (2015) suggests that it suffices to set the Wasserstein radius $\theta$ in an order of $N^{-1 / 2} \log (N)^{3 / 4}$ with respect to the sample size $N$ so that the type- $\infty$ Wasserstein ball $\mathcal{F}_{\infty}(\theta)$ contains the true distribution with high probability. That is to say, under the same condition, the worst-case regret is bounded from above by the same order. Neglecting the lower-order term, the worst-case regret decreases at the order of $1 / \sqrt{N}$.

\subsection{Efficient Golden Section Search}

In light of Lemma 1, Lemma 2, and Theorem 1, we can now quantify the worst-case regret of any order quantity $x \in[\underline{x}, \bar{x}]$ by computing of the two components

$$
\begin{aligned}
\bar{\gamma}(x) \triangleq \max _{x \leq y \leq \tilde{x}} \max _{\mathbb{P} \in \mathcal{F}_{\infty}(\theta)}\left\{\mathbb{E}_{\mathbb{P}}[\Pi(y, \tilde{u}, \tilde{v})-\Pi(x, \tilde{u}, \tilde{v})]\right\} & =\max _{x \leq y \leq \bar{x}} \frac{1}{N} \sum_{i \in[N]} \max _{\underline{u}_{i} \leq u \leq \bar{u}_{i}} \Lambda\left(x, y, u, \bar{v}_{i}\right) \\
\text { and } \underline{\gamma}(x) \triangleq \max _{\underline{x} \leq y \leq x} \max _{\mathbb{P} \in \mathcal{F}_{\infty}(\theta)}\left\{\mathbb{E}_{\mathbb{P}}[\Pi(y, \tilde{u}, \tilde{v})-\Pi(x, \tilde{u}, \tilde{v})]\right\} & =\max _{\underline{x} \leq y \leq x} \frac{1}{N} \sum_{i \in[N]} \max _{\underline{u}_{i} \leq u \leq \bar{u}_{i}} \Lambda\left(x, y, u, \underline{v}_{i}\right),
\end{aligned}
$$

both of which optimize $y$ to maximize the objective function $\max _{\mathbb{P} \in \mathcal{F}_{\infty}(\theta)}\left\{\mathbb{E}_{\mathbb{P}}[\Pi(y, \tilde{u}, \tilde{v})-\Pi(x, \tilde{u}, \tilde{v})]\right\}$ with good properties listed below. The proof follows straightforwardly from the summary in Table 1.

Corollary 1. For any $x \in[\underline{x}, \bar{x}]$, let us define two decision-dependent subsets $\left\{\bar{y}_{j}\right\}_{j \in[J]}=\{y \in$ $\left.\left\{\bar{v}_{i} / \bar{u}_{i}\right\}_{i \in[N]} \cup\left\{\bar{v}_{i} / \underline{u}_{i}\right\}_{i \in \mathcal{N}}: y \in[x, \bar{x}], \bar{y}_{1} \leq \bar{y}_{2} \leq \cdots \bar{y}_{J}\right\}$ and $\left\{\underline{y}_{k}\right\}_{k \in[K]}=\left\{y \in\left\{\underline{v}_{i} / \bar{u}_{i}\right\}_{i \in[N]} \cup\left\{\underline{v}_{i} / \underline{u}_{i}\right\}_{i \in \mathcal{N}}:\right.$ $\left.y \in[\underline{x}, x], \underline{y}_{1} \leq \underline{y}_{2} \leq \cdots \leq \underline{y}_{K}\right\}$. The function $\max _{\mathbb{P} \in \mathcal{F}_{\infty}(\theta)}\left\{\mathbb{E}_{\mathbb{P}}[\Pi(y, \tilde{u}, \tilde{v})-\Pi(x, \tilde{u}, \tilde{v})]\right\}$ is piecewise concave on the interval $[x, \bar{x}]$ with break points $\left\{\bar{y}_{j}\right\}_{j \in[J]}$ and is piecewise convex on the interval $[\underline{x}, x]$ with break points $\left\{\underline{y}_{k}\right\}_{k \in[K]}$. 
The properties above differ markedly from the known result when only demand is random: for any nonempty convex ambiguity set $\mathcal{F}$, the function $\max _{\mathbb{P} \in \mathcal{F}}\left\{\mathbb{E}_{\mathbb{P}}[\Pi(y, 1, \tilde{v})-\Pi(x, 1, \tilde{v})]\right\}$ is concave on both the intervals $y \geq x$ and $y \leq x$ (Perakis and Roels 2008, proposition 1). This difference provides another demonstration of random yield complex effects. We next leverage these properties to quantify the worst-case regret.

First, since for any fixed $x \in[\underline{x}, \bar{x}]$, the function $\frac{1}{N} \sum_{i \in[N]} \max _{\underline{u}_{i} \leq u \leq \bar{u}_{i}} \Lambda\left(x, y, u, \bar{v}_{i}\right)$ is piecewise concave on $y \in\left[x, \bar{y}_{1}\right] \cup\left[\bar{y}_{1}, \bar{y}_{2}\right] \cup \cdots \cup\left[\bar{y}_{J}, \bar{x}\right]$ with breaks points $\bar{y}_{j}, j \in[J]$, the problem determining $\bar{\gamma}(x)$ takes its maximum either at one of the boundary points of these intervals or at a point in a specified interval for which the derivative of the concave function $\frac{1}{N} \sum_{i \in[N]} \max _{\underline{u}_{i} \leq u \leq \bar{u}_{i}} \Lambda\left(x, y, u, \bar{v}_{i}\right)$ is zero. Let $\bar{y}_{0}=x$ and $\bar{y}_{J+1}=\bar{x}$. For any $j=0,1, \ldots, J$, according to Table 1 we have an explicit expression of that concave function in the interval $\left[\bar{y}_{j}, \bar{y}_{j+1}\right]$ and we can obtain the closed-form root $\bar{y}_{j}^{\star}$ of its derivative. We then update decision-dependent set with $\left\{\bar{y}_{j}\right\}_{j \in[J]} \cup\left\{\bar{y}_{j}^{\star}\right\}_{j \in \mathcal{J}}$, where $\mathcal{J}=\left\{j=0,1 \ldots, J: \bar{y}_{j}^{\star} \in\left[\bar{y}_{j}, \bar{y}_{j+1}\right]\right\}$, and we arrive at

$$
\bar{\gamma}(x)=\max _{y \in\left\{\bar{y}_{j}\right\}_{j \in[J]} \cup\left\{\bar{y}_{j}^{\star}\right\}_{j \in \mathcal{J}} \cup\{\bar{x}\} \cup\{x\}}\left\{\frac{1}{N} \sum_{i \in[N]} \max _{\underline{u}_{i} \leq u \leq \bar{u}_{i}} \Lambda\left(x, y, u, \bar{v}_{i}\right)\right\} .
$$

Second, since for any order quantity $x \in[\underline{x}, \bar{x}]$, the function $\frac{1}{N} \sum_{i \in[N]} \max _{\underline{u}_{i} \leq u \leq \bar{u}_{i}} \Lambda\left(x, y, u, \underline{v}_{i}\right)$ is piecewise convex on $y \in\left[\underline{x}_{1} \underline{y}_{1}\right] \cup\left[\underline{y}_{1}, \underline{y}_{2}\right] \cup \cdots \cup\left[\underline{y}_{K}, x\right]$ with breaks points $\underline{y}_{k}, k \in[K]$, the problem determing $\underline{\gamma}(x)$ takes its maximum at one of the boundary points of these intervals. This implies

$$
\underline{\gamma}(x)=\max _{y \in \underline{\mathcal{Y}}(x) \cup\{\underline{x}\} \cup\{x\}}\left\{\frac{1}{N} \sum_{i \in[N]} \max _{u_{i} \leq u \leq \bar{u}_{i}} \Lambda\left(x, y, u, \underline{v}_{i}\right)\right\} .
$$

Finally, to conclude the worst-case regret $\gamma(x)$ of an order quantity $x \in[\underline{x}, \bar{x}]$, we actually only need to compare values of at most $3 N+3|\mathcal{N}|+2$ point - at most $2(N+|\mathcal{N}|+1)$ points for solving problem (7) and at most $N+|\mathcal{N}|$ points for solving problem (8) - then, from among them, choose as the optimum that point with the largest objective value. In other words, $\gamma(x)=\max \{\bar{\gamma}(x), \underline{\gamma}(x)\}$. We summarize steps for computing $\gamma(x)$ in Algorithm 1. Recall that the minimax regret newsvendor model is a one-dimensional convex program in a bounded interval, its optimal order quantity can be efficiently determined by the standard golden section search method summarized in Algorithm 2.

REMARK 2. Steps in the golden section search are sufficient to recover the worst-case distribution associated with the optimal minimax regret order quantity $x_{\mathrm{R}}^{\star}$. Indeed, once we know the optimal auxiliary decision $y^{\star}$, then it is clear whether $y^{\star} \geq x_{\mathrm{R}}^{\star}$ or $y^{\star} \leq x_{\mathrm{R}}^{\star}$. By Lemmas 1 and 2 , for each $i \in[N]$ we can obtain the corresponding worst-case scenario $\left(u_{i}^{\star}, v_{i}^{\star}\right)$ on which the mixture component $\mathbb{P}_{i}^{\star}$ places a unit mass; that is, $\mathbb{P}_{i}^{\star}\left[\left(\tilde{u}_{i}, \tilde{v}_{i}\right)=\left(u_{i}^{\star}, v_{i}^{\star}\right)\right]=1$. The worst-case distribution $\mathbb{P}^{\star}$ in the type- $\infty$ Wasserstein ball is then given by $\mathbb{P}^{\star}=\frac{1}{N} \sum_{i \in[N]} \mathbb{P}_{i}^{\star}$, which (as its name implies) fulfills the condition

$$
\min _{x} \max _{\mathbb{P} \in \mathcal{F}_{\infty}(\theta)} \max _{y}\left\{\mathbb{E}_{\mathbb{P}}[\Pi(y, \tilde{u}, \tilde{v})-\Pi(x, \tilde{u}, \tilde{v})]\right\}=\mathbb{E}_{\mathbb{P} \star}\left[\Pi\left(y^{\star}, \tilde{u}, \tilde{v}\right)-\Pi\left(x_{\mathrm{R}}^{\star}, \tilde{u}, \tilde{v}\right)\right]
$$



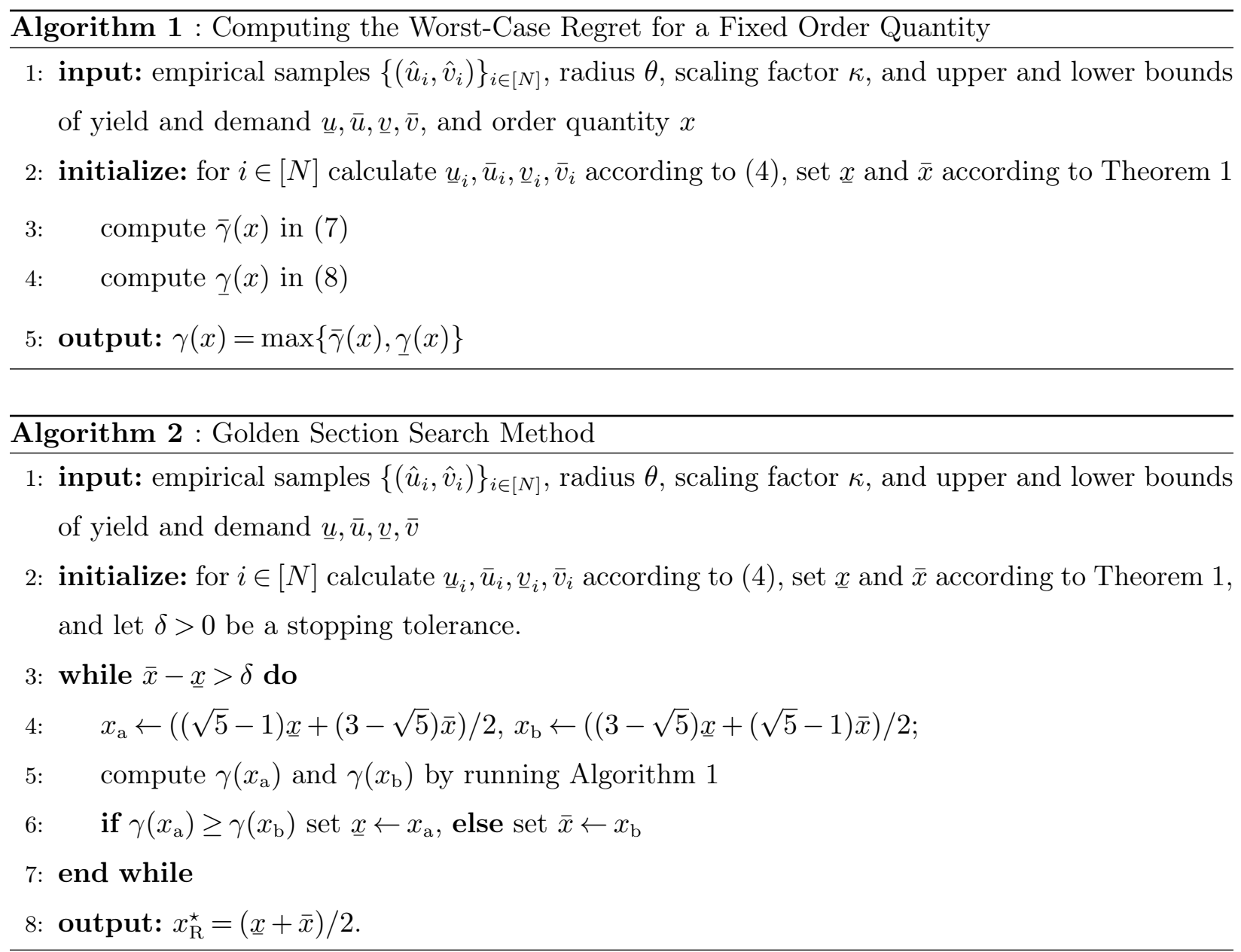

Such a worst-case distribution is interpreted as the most adversarial nature against which the optimal minimax regret order quantity $x_{\mathrm{R}}^{\star}$ hedges. From the practical perspective, the worst-case distribution $\mathbb{P}^{\star}$ could be helpful to perform targeted tests for different decisions that are not necessarily optimal under the minimax regret criterion.

\section{Special Cases of Minimax Regret Model}

Due to the more complex effects of random yield, the minimax regret newsvendor model with only yield randomness may not be more tractable than the general case with both demand and yield randomness. Nevertheless, most of our results about the general minimax regret model can be strengthened when only the demand is random. Another notable special case with stronger results is when our data-driven model recovers the traditional robust optimization approach as $N=1$.

\subsection{Model with Only Demand Randomness}

In the newsvendor model with only demand randomness, the yield is deterministic. In other words, the uncertainty set of the "random" yield is a singleton set $\mathcal{U}_{i}=\{1\}$ for every $i \in[N]$. Then the profit function can be simplified to read $V(x, v)=\Pi(x, 1, v)=(p-c) x-p(x-v)^{+}$. Given a type- $\infty$ 
Wasserstein ball of radius $\theta>0$ around the empirical distribution $\hat{\mathbb{P}}=\frac{1}{N} \sum_{i \in[N]} \delta_{\hat{v}_{i}}$ of the random demand, the minimax regret model with only demand randomness solves

$$
\min _{x} \max _{\mathbb{P} \in \mathcal{F}_{\infty}(\theta)} \max _{y}\left\{\mathbb{E}_{\mathbb{P}}[V(y, \tilde{v})-V(x, \tilde{v})]\right\}=\min _{x} \max _{y} \frac{1}{N} \sum_{i \in[N]} \max _{v \in \mathcal{V}_{i}}\{V(y, v)-V(x, v)\}
$$

here for each $i \in[N]$, the uncertainty set $\mathcal{V}_{i}=\left[\hat{v}_{i}-\theta, \hat{v}_{i}+\theta\right] \cap[\underline{v}, \bar{v}]=\left[\underline{v}_{i}, \bar{v}_{i}\right]$. Lemma 1 implies that

$$
\frac{1}{N} \sum_{i \in[N]} \max _{v \in \mathcal{V}_{i}}\{V(y, v)-V(x, v)\}= \begin{cases}\mathbb{E}_{\overline{\mathbb{P}}}[V(y, \tilde{v})-V(x, \tilde{v})] & \text { if } y \geq x \\ \mathbb{E}_{\mathbb{P}}[V(y, \tilde{v})-V(x, \tilde{v})] & \text { if } y \leq x,\end{cases}
$$

where $\overline{\mathbb{P}}=\frac{1}{N} \sum_{i \in[N]} \delta_{\bar{v}_{i}}$ and $\mathbb{P}=\frac{1}{N} \sum_{i \in[N]} \delta_{\underline{v}_{i}}$. Thus the minimax regret model becomes

$$
\min _{x} \max \left\{\max _{y \geq x} \mathbb{E}_{\overline{\mathbb{P}}}[V(y, \tilde{v})-V(x, \tilde{v})], \max _{y \leq x} \mathbb{E}_{\mathbb{P}}[V(y, \tilde{v})-V(x, \tilde{v})]\right\} .
$$

In the rest of this section, we show that most results about the general minimax regret model with both demand and yield randomness can be strengthened when only the demand is random.

Let $\bar{x}^{\star}$ (resp., $\left.\underline{x}^{\star}\right)$ be an optimal solution to the classical newsvendor problem $\max _{x} \mathbb{E}_{\overline{\mathbb{P}}}[V(x, \tilde{v})]$ (resp., $\max _{x} \mathbb{E}_{\mathbb{P}}[V(x, \tilde{v})]$ ); that is, let

$$
\bar{x}^{\star} \in \underset{x}{\arg \max } \mathbb{E}_{\overline{\mathbb{P}}}[V(x, \tilde{v})] \quad \text { and } \quad \underline{x}^{\star} \in \underset{x}{\arg \max } \mathbb{E}_{\mathbb{P}}[V(x, \tilde{v})],
$$

respectively. Also, let $\hat{x}^{\star} \in \arg \max _{x} \mathbb{E}_{\hat{\mathbb{P}}}[V(x, \tilde{v})]$ be an optimal solution to the empirical model. Then the following theorem states properties of an optimal order quantity $x_{\mathrm{R}}^{\star}$ in the minimax regret model (9). The results are similar to those in Theorem 1 and Proposition 1, yet are more explicit because only the demand is random.

TheOREM 2. The minimax regret model (9) with only demand randomness is equivalent to

$$
\min _{x \in\left[\underline{x}^{\star}, \bar{x}^{\star}\right]} \max \left\{\mathbb{E}_{\overline{\mathbb{P}}}\left[V\left(\bar{x}^{\star}, \tilde{v}\right)-V(x, \tilde{v})\right], \mathbb{E}_{\mathbb{P}}\left[V\left(\underline{x}^{\star}, \tilde{v}\right)-V(x, \tilde{v})\right]\right\} .
$$

There is an optimal minimax regret order quantity $x_{\mathrm{R}}^{\star}$ residing in the interval $\left[\underline{x}^{\star}, \bar{x}^{\star}\right]$ and satisfying

$$
\sum_{i \in[N]}\left(x_{\mathrm{R}}^{\star}-\underline{v}_{i}\right)^{+}-\sum_{i \in[N]}\left(x_{\mathrm{R}}^{\star}-\bar{v}_{i}\right)^{+}=\frac{(p-c) N}{p}\left(\bar{x}^{\star}-\underline{x}^{\star}\right) .
$$

REMARK 3. Suppose that the type- $\infty$ Wasserstein ball does not contain any support information; that is, $\underline{v}=-\infty$ and $\bar{v}=\infty$. This implies $\bar{v}_{i}=\hat{v}_{i}+\theta$ and $\underline{v}_{i}=\hat{v}_{i}-\theta$ for each $i \in[N]$ as well as $\bar{x}^{\star}=\hat{x}^{\star}+\theta$ and $\underline{x}^{\star}=\hat{x}^{\star}-\theta$. Then we can simplify the optimality condition (12) as $\sum_{i \in[N]}\left(x_{\mathrm{R}}^{\star}+\theta-\right.$ $\left.\hat{v}_{i}\right)^{+}-\sum_{i \in[N]}\left(x_{\mathrm{R}}^{\star}-\theta-\hat{v}_{i}\right)^{+}=\frac{2(p-c) N}{p} \theta$.

Theorem 2 also gives the interval $\left[\underline{x}^{\star}, \bar{x}^{\star}\right] \subseteq\left[\hat{x}^{\star}-\theta, \hat{x}^{\star}+\theta\right]$ in which $x_{\mathrm{R}}^{\star}$ lies. This property readily establishes a straightforward convergence of the optimal minimax regret order quantity to the true optimal one that maximizes the expected profit under the true demand distribution. The proof follows from the inclusion $x^{\star} \in\left[\hat{x}^{\star}-\theta, \hat{x}^{\star}+\theta\right]$ and the triangle inequality. 
COROLlary 2. Let $\mathbb{P}_{\mathrm{o}}$ be the true demand distribution that generates the empirical samples $\hat{v}_{1}, \ldots, \hat{v}_{N}$, and let $x_{\mathrm{o}}^{\star}$ be the optimal decision that maximizes the expected profit under the true demand distribution $\mathbb{P}_{\mathrm{o}}$, that is, $x_{\mathrm{o}}^{\star} \in \arg \max _{x} \mathbb{E}_{\mathbb{P}_{\mathrm{o}}}[V(x, \tilde{v})]$. Then $\left|x_{\mathrm{R}}^{\star}-x_{\mathrm{o}}^{\star}\right| \leq\left|\hat{x}^{\star}-x_{\mathrm{o}}^{\star}\right|+\theta$.

REMARK 4. Although the loose triangle inequality may imply the convergence could be weak, Corollary 2 is of interest in its own right. Recall that $\hat{x}^{\star}$ and $x_{\mathrm{o}}^{\star}$ are $\frac{p-c}{p}$-quantiles of $\hat{\mathbb{P}}$ and $\mathbb{P}_{\mathrm{o}}$, respectively. Corollary 2 provides a rough estimation that, as compared with the empirical model, the minimax regret model further pays (at most) an additional price $\theta$ of robustness in terms of distance to the true optimal order quantity. Therefore, existing convergence of $\hat{x}^{\star}$ to $x_{\mathrm{o}}^{\star}$, which itself has been widely studied, ${ }^{2}$ can be seemlessly applied to $x_{\mathrm{R}}^{\star}$. Indeed, $\hat{x}^{\star}$ converges uniformly and with an exponential rate to $x_{\mathrm{o}}^{\star}$ (Serfling 2009, section 2.3). Hence, under the same condition in Remark 1, the distance from $x_{\mathrm{R}}^{\star}$ to $x_{\mathrm{o}}^{\star}$ decreases (at least) at the order of $1 / \sqrt{N}$ as the sample size $N$ grows. $\diamond$

REMARK 5. Corollary 2 neither shows statistical advantages of the minimax regret model, nor concludes that the theoretically error estimation of the robust quantile can be better than the empirical. However, it has been numerically and theoretically shown that a distributionally robust model typically has better out-of-sample performances than the empirical counterpart (see, e.g., Mohajerin Esfahani and Kuhn 2018).

Lastly, we bound (from above) the regret in the minimax regret model. Without loss of generality, we assume that $\hat{v}^{1} \leq \hat{v}^{2} \leq \cdots \leq \hat{v}^{N}$. It then follows from the optimality condition (2) with $\tilde{u}=1$ almost surely that $\hat{x}^{\star}=\hat{v}_{k^{\star}}$; here $k^{\star}$ is the smallest index $k \in[N]$ that satisfies $\sum_{i=k+1}^{N} p \leq c N$, (i.e., $p k \geq(p-c) N)$. With empirical samples $\left\{\hat{v}_{i}\right\}_{i \in[N]}$ of such an order, we have $\bar{x}^{\star}=\bar{v}_{k^{\star}}$ and $\underline{x}^{\star}=\underline{v}_{k^{\star}}$-which satisfy $0 \leq \bar{x}^{\star}-\underline{x}^{\star} \leq 2 \theta$. This result is summarized as follows.

Proposition 3. In the minimax regret model (11) with only demand randomness, the optimal (i.e., the least) worst-case regret of is bounded from above by

$$
2 \theta \min \left\{\frac{p j}{N}-(p-c),(p-c)-\frac{p \ell}{N}\right\}
$$

Here we let $j$ be the smallest index in $\left\{k^{\star}+1, \ldots, N\right\}$ that satisfies $\underline{v}_{j}-\underline{v}_{k^{\star}} \geq 2 \theta$ and let $\ell$ be the largest index in $\left\{1, \ldots, k^{\star}-1\right\}$ that satisfies $\bar{v}_{k^{\star}}-\bar{v}_{\ell} \geq 2 \theta$. In particular, we take $j=k^{\star}$ if $k^{\star}=N$ or if $\underline{v}_{k^{\star}+1}-\underline{v}_{k^{\star}} \geq 2 \theta$ whereas $\ell=k^{\star}-1$ if $k^{\star}=1$ or $\bar{v}_{k^{\star}}-\bar{v}_{k^{\star}-1} \geq 2 \theta$.

Under the same condition in Remark 1, the worst-case regret decreases in the order of $1 / \sqrt{N}$.

\footnotetext{
${ }^{2}$ For example, Levi et al. (2007) study the newsvendor model with only demand randomness under the discrete empirical distribution $\hat{\mathbb{P}}$ and establish the convergence of $\hat{x}^{\star}$ to $x_{\mathrm{o}}^{\star}$. Levi et al. (2015) recently revisit the heuristic in Levi et al. (2007) and provide new bounds and insights.
} 


\subsection{Traditional Robust Perspective}

When $N=1$, the type- $\infty$ Wasserstein ball reduces to the an uncertainty set $\mathcal{F}_{\infty}(\theta)=\left\{\mathbb{P} \in \mathcal{P}\left(\mathbb{R}^{2}\right) \mid\right.$ $\left.\mathbb{P}\left[(\tilde{u}, \tilde{v}) \in \mathcal{U}_{1} \times \mathcal{V}_{1}\right]=1\right\}$ with $\mathcal{U}_{1}=\left[\underline{u}_{1}, \bar{u}_{1}\right]$ and $\mathcal{V}_{1}=\left[\underline{v}_{1}, \bar{v}_{1}\right]$. The distributionally robust perspective, which considers the extreme-case expected performance within the ambiguity set, reduces to the traditional robust perspective that considers the extreme scenario within the uncertainty set. In this situation, the optimal decision in the minimax regret model can be derived in a closed form.

Proposition 4. Let $N=1$ and suppose that $\mathcal{U}_{1}=\left[\underline{u}_{1}, \bar{u}_{1}\right]$, and $\mathcal{V}_{1}=\left[\underline{v}_{1}, \bar{v}_{1}\right]$. Then the optimal order quantity $x_{\mathrm{R}}^{\star}$ in the minimax regret newsvendor model is given by $x_{\mathrm{R}}^{\star}=\frac{(p-c) \bar{v}_{1}+c \underline{v}_{1}}{(p-c) \underline{u}_{1}+c \bar{u}_{1}}$, whose associated worst-case regret amounts to $\frac{c(p-c)\left(\bar{u}_{1} \bar{v}_{1}-\underline{u}_{1} \underline{v}_{1}\right)}{p}$.

When there is only demand randomness, i.e., $\underline{u}_{1}=\bar{u}_{1}=1$, the optimal minimax regret order quantity takes $x_{\mathrm{R}}^{\star}=\left((p-c) \bar{v}_{1}+c \underline{v}_{1}\right) / p$, associated with a worst-case regret $\left(c(p-c)\left(\bar{v}_{1}-\underline{v}_{1}\right)\right) / p$. Indeed, this is an existing result on the optimal minimax regret order quantity when only the range (or support) of the demand distribution is known; see e.g., theorem 1 in Perakis and Roels (2008).

When there is only yield randomness, i.e., $\underline{v}_{1}=\bar{v}_{1}=v$, the optimal minimax regret order quantity takes $x_{\mathrm{R}}^{\star}=p v /\left((p-c) \bar{v}_{1}+c \underline{v}_{1}\right)$, which, indeed, coincides with the optimal maximin order quantity. To see this, we consider the minimax regret model

$$
\min _{x} \max _{u \in \mathcal{U}_{1}} \max _{y}\left\{\left((p-c) u y-p(u y-v)^{+}\right)-\left((p-c) u x-p(u x-v)^{+}\right)\right\}
$$

and observe that for any $u \in \mathcal{U}_{1}$, the optimal decision for the inner maximization problem over $y$ is $v / u$. Thus the minimax regret model can be simplified into

$$
\min _{x} \max _{u \in \mathcal{U}_{1}}\left\{(p-c) v-(p-c) u x+p(u x-v)^{+}\right\}=(p-c) v-\max _{x} \min _{u \in \mathcal{U}_{1}}\left\{(p-c) u x-p(u x-v)^{+}\right\}
$$

here the maximin problem on the right-hand side of the identity is, in essence, the maximin model. It is worth noting that the least worst-case regret is equal to the globally maximal profit (i.e., under the perfect yield condition that all orders are fulfilled) less the optimal robust profit.

\section{Hurwicz Criterion Model}

In this section, we investigate the newsvendor model with both demand and yield randomness under the Hurwicz criterion, which characterizes the decision-maker's perception of distributional ambiguity, ranging from the most optimistic one to the most pessimistic one.

With the level of optimism $\lambda \in[0,1]$ that combines the most optimistic perception of the maximax model and the most pessimistic perception of the maximin model, the Hurwicz criterion solves

$$
\max _{x \geq 0}\left\{\lambda \cdot \max _{\mathbb{P} \in \mathcal{F}_{\infty}(\theta)} \mathbb{E}_{\mathbb{P}}[\Pi(x, \tilde{u}, \tilde{v})]+(1-\lambda) \cdot \min _{\mathbb{P} \in \mathcal{F}_{\infty}(\theta)} \mathbb{E}_{\mathbb{P}}[\Pi(x, \tilde{u}, \tilde{v})]\right\},
$$


which seeks a maximal weighted average of the best- and worst-case expected profits within the type- $\infty$ Wasserstein ball $\mathcal{F}(\theta)$. The Hurwicz criterion model recovers the maximax (resp., maximin) model when $\lambda=1$ (resp., $\lambda=0$ ). It admits a scalable linear program reformulation that incorporates $2 N$ decision variables (apart from the order quantity) and $5 N$ constraints.

Proposition 5. The Hurwicz criterion newsvendor model (14) is equivalent to the problem

$$
\max _{x \geq 0}\left\{\frac{\lambda}{N} \sum_{i \in[N]} \min \left\{(p-c) \bar{u}_{i} x,(p-c) \bar{v}_{i}, p \bar{v}_{i}-c \underline{u}_{i} x\right\}+\frac{1-\lambda}{N} \sum_{i \in[N]} \min \left\{(p-c) \underline{u}_{i} x, p \underline{v}_{i}-c \bar{u}_{i} x\right\}\right\},
$$

which can be reformulated into the following linear program:

$$
\begin{aligned}
& \max _{x, \boldsymbol{\phi}, \boldsymbol{\psi}} \frac{\lambda}{N} \sum_{i \in[N]} \phi_{i}+\frac{1-\lambda}{N} \sum_{i \in[N]} \psi_{i} \\
& \text { s.t. } \phi_{i} \leq(p-c) \bar{u}_{i} x \quad \forall i \in[N] \\
& \phi_{i} \leq(p-c) \bar{v}_{i} \quad \forall i \in[N] \\
& \phi_{i} \leq p \bar{v}_{i}-c \underline{u}_{i} x \quad \forall i \in[N] \\
& \psi_{i} \leq(p-c) \underline{u}_{i} x \quad \forall i \in[N] \\
& \psi_{i} \leq p \underline{v}_{i}-c \bar{u}_{i} x \quad \forall i \in[N] \\
& x \in \mathbb{R}_{+}, \boldsymbol{\phi} \in \mathbb{R}^{N}, \boldsymbol{\psi} \in \mathbb{R}^{N} \text {. }
\end{aligned}
$$

The objective function of reformulation (15) is a weighted average of the best- and worst-case expected profits - each of which consists of a collection of concave piecewise affine functions. For the $i^{\text {th }}$ component in the best-case collection, the corresponding three pieces intersect at $\bar{v}_{i} / \underline{u}_{i}$ and $\bar{v}_{i} / \bar{u}_{i}$ if $i \in \mathcal{N}$ but intersect only at $\bar{v}_{i} / \bar{u}_{i}$ if $i \in[N] \backslash \mathcal{N}$. Similarly, for each $i \in[N]$, the corresponding two pieces of the $i^{\text {th }}$ component in the worst-case collection intersect at $p \underline{v}_{i} /\left((p-c) \underline{u}_{i}+c \bar{u}_{i}\right)$. Hence the objective function of the Hurwicz criterion model is actually a concave piecewise affine function of the order quantity $x$ with a total number $2 N+|\mathcal{N}| \leq 3 N$ of break points:

$$
\frac{\bar{v}_{1}}{\underline{u}_{1}}, \frac{\bar{v}_{2}}{\underline{u}_{2}}, \ldots, \frac{\bar{v}_{|\mathcal{N}|}}{\underline{u}_{|\mathcal{N}|}}, \frac{\bar{v}_{1}}{\bar{u}_{1}}, \frac{\bar{v}_{2}}{\bar{u}_{2}}, \ldots, \frac{\bar{v}_{N}}{\bar{u}_{N}}, \frac{p \underline{v}_{1}}{(p-c) \underline{u}_{1}+c \bar{u}_{1}}, \frac{p \underline{v}_{2}}{(p-c) \underline{u}_{2}+c \bar{u}_{2}}, \ldots, \frac{p \underline{v}_{N}}{(p-c) \underline{u}_{N}+c \bar{u}_{N}} .
$$

These break points are, in fact, all possible values of the $x$-component of extreme points of the linear program (16). It follows that one of them must be an optimal order quantity in the Hurwicz criterion newsvendor model (14). Moreover, that optimal order quantity can be characterized by an optimality condition. These results are summarized in our next proposition.

Proposition 6. There is an optimal order quantity $x_{\mathrm{H}}^{\star}$ in the Hurwicz criterion newsvendor model (14) that takes its value from one of the $2 N+|\mathcal{N}|$ break points in (17). Furthermore, there exists an optimal order quantity for which $x_{\mathrm{H}}^{\star}=z_{k^{\star}}$; here $z_{1} \leq z_{2} \leq \cdots \leq z_{2 N+|\mathcal{N}|}$ is an ascending permutation of break points in (17) and $k^{\star}$ is the smallest index in $\{1,2, \ldots, 2 N+|\mathcal{N}|\}$ such that

$$
\lambda \sum_{i \in[N] \backslash \mathcal{J}_{k^{\star}}}(p-c) \bar{u}_{i}+(1-\lambda) \sum_{i \in[N] \backslash \mathcal{M}_{k^{\star}}}(p-c) \underline{u}_{i} \leq \lambda \sum_{i \in \mathcal{L}_{k^{\star}}} c \underline{u}_{i}+(1-\lambda) \sum_{i \in \mathcal{M}_{k^{\star}}} c \bar{u}_{i} .
$$


For any $k=1,2, \ldots, 2 N+|\mathcal{N}|$, we have the three index sets

$$
\mathcal{J}_{k}=\left\{i \in[N]: \frac{\bar{v}_{i}}{\bar{u}_{i}} \leq z_{k}\right\}, \mathcal{L}_{k}=\left\{i \in[N]: \frac{\bar{v}_{i}}{\underline{u}_{i}} \leq z_{k}\right\}, \mathcal{M}_{k}=\left\{i \in[N]: \frac{p \underline{v}_{i}}{(p-c) \underline{u}_{i}+c \bar{u}_{i}} \leq z_{k}\right\} .
$$

Proposition 6 recovers the known characterization (2) of the optimal order quantity in the stochastic newsvendor model with both demand and yield randomness under a known discrete distribution (see Appendix B for the details). Moreover, since, in general, $\lambda \in[0,1]$ and $\theta>0$, we can extend the characterization to consider the Hurwicz criterion under distributional ambiguity.

\subsection{Comparison with Minimax Regret Model: Only Demand Randomness}

In the case of only demand randomness, given $x$, the profit function $V(x, v)=(p-c) x-p(x-v)^{+}$is nondecreasing in $v$. Thus for each $i \in[N]$ we have $\min _{v \in \mathcal{V}_{i}}\left\{(p-c) x-p(x-v)^{+}\right\}=(p-c) x-p(x-$ $\left.\underline{v}_{i}\right)^{+}$while $\max _{v \in \mathcal{V}_{i}}\left\{(p-c) x-p(x-v)^{+}\right\}=(p-c) x-p\left(x-\bar{v}_{i}\right)^{+}$. The implication is that the maximin (maximax) model, taking $\lambda=0(\lambda=1)$ in the Hurwicz criterion model, can be reformulated as

$$
\begin{gathered}
\max _{x} \frac{1}{N} \sum_{i \in[N]}\left\{(p-c) x-p\left(x-\underline{v}_{i}\right)^{+}\right\}=\max _{x} \mathbb{E}_{\mathbb{P}}[V(x, \tilde{v})] \\
\left(\max _{x} \frac{1}{N} \sum_{i \in[N]}\left\{(p-c) x-p\left(x-\bar{v}_{i}\right)^{+}\right\}=\max _{x} \mathbb{E}_{\overline{\mathbb{P}}}[V(x, \tilde{v})]\right),
\end{gathered}
$$

where we recall $\mathbb{P}=\frac{1}{N} \sum_{i \in[N]} \delta_{\underline{v}_{i}}\left(\overline{\mathbb{P}}=\frac{1}{N} \sum_{i \in[N]} \delta_{\bar{v}_{i}}\right)$. That is to say, the optimal maximin order quantity takes $x_{\text {maximin }}^{\star}=\underline{x}^{\star}$ while the optimal maximax order quantity takes $x_{\text {maximax }}^{\star}=\bar{x}^{\star}$, where $\underline{x}^{\star}, \bar{x}^{\star}$ are defined in (10). Since the optimal minimax regret order quantity $x_{\mathrm{R}}^{\star}$ satisfies $x_{\mathrm{R}}^{\star} \in\left[\underline{x}^{\star}, \bar{x}^{\star}\right]$, we conclude that, when there is only demand randomness, the minimax regret model always overorders (underorders) in comparison with the maximin (maximax) model, regardless of the number of empirical samples. The optimal minimax regret order quantity in the model, compared with that in the maximin or maximax model, is less extreme and therefore more meaningful. On the other hand, it is arguable that how a decision-maker can precisely articulate the level of optimism $\lambda$ while avoiding conservative pessimism or aggresive optimism.

\subsection{Comparison with Minimax Regret Model: Traditional Robust Perspective}

Much as in Section 4.2, if we focus on the special case of $N=1$ then the optimal order quantity in the Hurwicz criterion model can be derived in closed form.

Proposition 7. Let $N=1$ and $\mathcal{U}_{1}=\left[\underline{u}_{1}, \bar{u}_{1}\right]$, and suppose that $\mathcal{V}_{1}=\left[\underline{v}_{1}, \bar{v}_{1}\right]$ and $\lambda \in[0,1]$. Then the optimal order quantity $x_{\mathrm{H}}^{\star}$ in the Hurwicz criterion newsvendor model is given by

$$
x_{\mathrm{H}}^{\star}= \begin{cases}\frac{\bar{v}_{1}}{\bar{u}_{1}} & \text { if } p\left(\underline{v}_{1} \bar{u}_{1}-\bar{v}_{1} \underline{u}_{1}\right)-c\left(\bar{v}_{1} \bar{u}_{1}-\bar{v}_{1} \underline{u}_{1}\right) \leq 0 \text { and } \lambda p \geq c, \\ \frac{(p-c) \underline{u}_{1}+c \bar{u}_{1}}{(p} & \text { otherwise. }\end{cases}
$$


For the extreme optimism (i.e., $\lambda=1$ ), Proposition 7 suggests that the optimal order quantity $x_{\text {maximax }}^{\star}$ in the maximax model can be either $\bar{v}_{1} / \bar{u}_{1}$ or $p \underline{v}_{1} /\left[(p-c) \underline{u}_{1}+c \bar{u}_{1}\right]$. Indeed, it can be easily verified that $x_{\text {maximax }}^{\star}$ can take any value in the interval $\left[\bar{v}_{1} / \bar{u}_{1}, \bar{v}_{1} / \underline{u}_{1}\right]$. For the extreme pessimism (i.e., $\lambda=0$ ), Proposition 7 suggests that the optimal order quantity $x_{\text {maximin }}^{\star}$ in the maximin model is $p \underline{v}_{1} /\left[(p-c) \underline{u}_{1}+c \bar{u}_{1}\right]$. This result is consistent with our intuition that the more optimistic model would call for a larger order; indeed, as $p \underline{v}_{1} /\left[(p-c) \underline{u}_{1}+c \bar{u}_{1}\right] \leq \bar{v}_{1} / \bar{u}_{1}$, any optimal order quantity in the maximax model is no smaller than that in the maximin model.

By Propositions 4 and 7, the optimal order quantity in the minimax regret model is larger than in the maximin model:

$$
x_{\mathrm{R}}^{\star}=\frac{p \underline{v}_{1}}{(p-c) \underline{u}_{1}+c \bar{u}_{1}}+\frac{(p-c)\left(\bar{v}_{1}-\underline{v}_{1}\right)}{(p-c) \underline{u}_{1}+c \bar{u}_{1}} \geq \frac{p \underline{v}_{1}}{(p-c) \underline{u}_{1}+c \bar{u}_{1}}=x_{\text {maximin }}^{\star} .
$$

This result is consistent with that in the established case of random demand (for which the optimal order quantity in the minimax regret model is always no smaller than that in the maximin model).

\section{Numerical Experiments}

We present the encouraging out-of-sample performance of our data-driven minimax regret model over alternative data-driven models based on Hurwicz decision criterion and its variants (Section 6.1), over a minimax regret model in Perakis and Roels (2008) that is based on partial statistical information on moments (Section 6.2), and in a test where the testing distribution differs from the training one (Section 6.3). Throughout these experiments, we use the same parameters as Schweitzer and Cachon (2000) and Käki et al. (2015): the unit price is $p=12$, while the unit cost may take $c=3$ (high profit margin), $c=6$ (median profit margin), or $c=9$ (low profit margin). In our numerical experiments, the data-driven minimax regret model qualitatively shows strong competence.

1. In 33 out 36 (18 out of 36 ) situations in Section 6.1 (Section 6.3), it performs the best among considered alternatives therein.

2. For other situations when the data-driven minimax regret model may not be the best (e.g., several situations in Section $6.2^{3}$ ), its performance gap to the true optimum with perfect information is typically not larger than $5 \%$, except for some extreme situations with scarce training samples (Tables 5 and 6 with $N=5$ ).

3. The theoretical convergence of the data-driven minimax regret model to the true optimum as the sample size grows ensures that our proposed model will be clearly more advantageous once the data are abundant (e.g., $N>50)$.

\footnotetext{
${ }^{3}$ We will explain why the data-driven minimax regret model should not be expected to outperform the considered alternatives.
} 
4. It is worthy of mentioning that the numerical insights in Section 6.2 and Section 6.3 are for a fair comparison where only demand is uncertain, because it remains unclear how moment-based models can handle yield randomness. Thus, we recommend using our proposed data-driven minimax regret model whenever there is yield randomness on the supply side.

5. The golden section search always finds the optimal minimax regret order quantity within tenth of a second, indicating negligible computational efforts.

\subsection{Comparisons of Decision Criteria with Both Demand and Yield Randomness}

We study the newsvendor model with both demand and yield randomness to compare different decision criteria. The random demand is uniformly distributed in $[\underline{v}, \bar{v}] \triangleq[0,300]$ and the random yield is uniformly supported on $[\underline{u}, \bar{u}] \triangleq[0.4,1]$. The demand and yield dependency is characterized through the Farlie-Gumbel-Morgenstern (FGM) copula (Cossette et al. 2008), which, given uniform marginals $\tilde{u} \sim \mathbb{U}[\underline{u}, \bar{u}]$ and $\tilde{v} \sim \mathbb{U}[\underline{v}, \bar{v}]$, and a parameter $\eta \in[-1,1]$, is formally defined as

$$
C(u, v) \triangleq \frac{(u-\underline{u})(v-\underline{v})[a b+\eta(\bar{u}-u)(\bar{v}-v)]}{a^{2} b^{2}} .
$$

Here, $a \triangleq \bar{u}-\underline{u}, b \triangleq \bar{v}-\underline{v}$, and $\eta$ represents dependency strength; in other words, the linear correlation coefficient of $\tilde{u}$ and $\tilde{v}$ is $\eta / 3$. In fact, independent demand and yield may not always be realistic: they are negatively correlated if the supply capacity is constrained while being positively correlated if suppliers can flexibly increase the capacity (see detailed motivating examples in Käki et al. 2015). The joint probability density function of $(\tilde{u}, \tilde{v})$ with FGM copula takes

$$
f(u, v) \triangleq \frac{\partial C(u, v)}{\partial u \partial v}=\frac{a b+\eta(\bar{u}+\underline{u}-2 u)(\bar{v}+\underline{v}-2 v)}{a^{2} b^{2}} .
$$

Stochastic newsvendor model (1) admits an analytical solution when the true joint demand and yield distribution is specified by the FGM copula; see Appendix C for the detailed derivations.

We randomly generate $N$ (training) empirical samples from the true joint demand and yield distribution $\mathbb{P}_{\mathrm{o}}$ with FGM copula. For the demonstration purpose, we construct a type- $\infty$ Wasserstein ball $\mathcal{F}_{\infty}(\theta)$ of $\operatorname{radius}^{4} \theta=2(\bar{v}-\underline{v}) N^{-1}=200 / \sqrt{N}$ that will diminish to zero as the sample size $N$ increases and we set accordingly a scaling factor $\kappa=b / a=500$. We first solve the optimal order quantities under the minimax regret, maximax, maximin, and Hurwicz ${ }^{5}$ decision criteria, as well as a heuristic that averages the optimal maximax and maximin order quantities. Then we compare their out-of-sample performances with the true optimal order quantity, using $10^{7}$ (testing) samples drawn randomly from the true underlying distribution. For each considered combination of profit

\footnotetext{
${ }^{4}$ In practice, one can also use cross validation to find a more appropriate Wasserstein radius (see e.g. Mohajerin Esfahani and Kuhn 2018, Xie et al. 2021). However, in our numerical experiments, we find $\theta=200 / \sqrt{N}$ suffices because we do not observe any qualitative changes in the results.

${ }^{5}$ We use 5 -fold cross validation on the training samples to select the level of optimism $\lambda$ from $\{0,0.1, \cdots, 0.9,1\}$.
} 


\begin{tabular}{cccccccccc}
\hline \hline$(\eta, N)$ & $(-1,5)$ & $(-1,15)$ & $(-1,50)$ & $(0,5)$ & $(0,15)$ & $(0,50)$ & $(1,5)$ & $(1,15)$ & $(1,50)$ \\
\hline regret & 0.957 & 0.977 & 0.993 & 0.962 & 0.984 & 0.995 & 0.970 & 0.988 & 0.996 \\
& {$[1.022]$} & {$[1.002]$} & {$[1.006]$} & {$[1.018]$} & {$[1.002]$} & {$[1.000]$} & {$[1.018]$} & {$[1.006]$} & {$[0.999]$} \\
Hurwicz & 0.927 & 0.960 & 0.989 & 0.944 & 0.973 & 0.992 & 0.957 & 0.981 & 0.984 \\
& {$[0.999]$} & {$[1.004]$} & {$[1.004]$} & {$[0.989]$} & {$[0.994]$} & {$[0.998]$} & {$[0.981]$} & {$[0.994]$} & {$[0.999]$} \\
maximax & 0.927 & 0.953 & 0.980 & 0.926 & 0.958 & 0.984 & 0.931 & 0.965 & 0.986 \\
& {$[1.165]$} & {$[1.130]$} & {$[1.096]$} & {$[1.124]$} & {$[1.098]$} & {$[1.066]$} & {$[1.085]$} & {$[1.070]$} & {$[1.050]$} \\
maximin & 0.714 & 0.879 & 0.971 & 0.727 & 0.896 & 0.972 & 0.731 & 0.907 & 0.975 \\
& {$[0.661]$} & {$[0.747]$} & {$[0.870]$} & {$[0.678]$} & {$[0.769]$} & {$[0.881]$} & {$[0.678]$} & {$[0.787]$} & {$[0.896]$} \\
heuristic & 0.953 & 0.972 & 0.992 & 0.951 & 0.978 & 0.994 & 0.951 & 0.982 & 0.995 \\
& {$[0.916]$} & {$[0.956]$} & {$[0.993]$} & {$[0.917]$} & {$[0.960]$} & {$[0.986]$} & {$[0.908]$} & {$[0.959]$} & {$[0.987]$} \\
\hline \hline
\end{tabular}

Table 2 Mean of profit distribution for high profit margin.

\begin{tabular}{ccccccccccc}
\hline \hline$(\eta, N)$ & $(-1,5)$ & $(-1,15)$ & $(-1,50)$ & $(0,5)$ & $(0,15)$ & $(0,50)$ & $(1,5)$ & $(1,15)$ & $(1,50)$ \\
\hline \multirow{2}{*}{ regret } & 0.885 & 0.937 & 0.978 & 0.914 & 0.958 & 0.982 & 0.946 & 0.967 & 0.987 \\
& {$[1.177]$} & {$[1.098]$} & {$[1.042]$} & {$[1.093]$} & {$[1.034]$} & {$[1.027]$} & {$[1.023]$} & {$[1.019]$} & {$[1.013]$} \\
Hurwicz & 0.730 & 0.897 & 0.971 & 0.789 & 0.929 & 0.975 & 0.838 & 0.944 & 0.982 \\
& {$[1.188]$} & {$[1.068]$} & {$[1.019]$} & {$[1.108]$} & {$[1.016]$} & {$[1.017]$} & {$[1.050]$} & {$[1.012]$} & {$[1.007]$} \\
maximax & 0.391 & 0.709 & 0.896 & 0.501 & 0.779 & 0.911 & 0.608 & 0.811 & 0.924 \\
& {$[1.850]$} & {$[1.590]$} & {$[1.353]$} & {$[1.626]$} & {$[1.439]$} & {$[1.291]$} & {$[1.466]$} & {$[1.355]$} & {$[1.241]$} \\
maximin & 0.585 & 0.709 & 0.905 & 0.579 & 0.721 & 0.913 & 0.563 & 0.754 & 0.922 \\
& {$[0.503]$} & {$[0.576]$} & {$[0.708]$} & {$[0.496]$} & {$[0.564]$} & {$[0.726]$} & {$[0.490]$} & {$[0.587]$} & {$[0.739]$} \\
heuristic & 0.921 & 0.937 & 0.977 & 0.942 & 0.956 & 0.981 & 0.954 & 0.964 & 0.986 \\
& {$[1.162]$} & {$[1.069]$} & {$[1.033]$} & {$[1.062]$} & {$[0.997]$} & {$[1.015]$} & {$[0.983]$} & {$[0.979]$} & {$[1.001]$} \\
\hline \hline
\end{tabular}

Table 3 Mean of profit distribution for median profit margin.

margin (specified by c), demand and yield dependency (specified by $\eta$ ), and training sample size $N$, the data-driven experiment is replicated 1000 times. For each approach we report mean and standard deviation of its profit distribution based on average results among these 1000 random trials, where we have normalized the expected profit and standard deviation under the true optimal order quantity to unity. The complete results of all considered combinations are relegated to Appendix D.

\section{Advantages of minimax regret model}

Tables 2, 3, and 4 show that compared with the maximax, maximin and Hurwicz decision criteria as well as the heuristic, the minimax regret approach is most likely to perform the best: in 33 out of 36 considered combinations it attains the highest expected profit. This is because the minimax regret 


\begin{tabular}{cccccccccc}
\hline \hline$(\eta, N)$ & $(-1,5)$ & $(-1,15)$ & $(-1,50)$ & $(0,5)$ & $(0,15)$ & $(0,50)$ & $(1,5)$ & $(1,15)$ & $(1,50)$ \\
\hline regret & 0.563 & 0.797 & 0.937 & 0.706 & 0.861 & 0.947 & 0.827 & 0.892 & 0.956 \\
& {$[1.780]$} & {$[1.419]$} & {$[1.132]$} & {$[1.522]$} & {$[1.259]$} & {$[1.086]$} & {$[1.262]$} & {$[1.169]$} & {$[1.071]$} \\
Hurwicz & 0.357 & 0.717 & 0.924 & 0.465 & 0.762 & 0.929 & 0.592 & 0.808 & 0.942 \\
& {$[1.817]$} & {$[1.291]$} & {$[1.064]$} & {$[1.569]$} & {$[1.175]$} & {$[1.051]$} & {$[1.311]$} & {$[1.145]$} & {$[1.045]$} \\
maximax & -3.460 & -0.687 & 0.561 & -271.1 & -0.345 & 0.623 & -1.809 & -0.129 & 0.664 \\
& {$[4.521]$} & {$[3.065]$} & {$[1.972]$} & {$[3.788]$} & {$[2.675]$} & {$[1.849]$} & {$[3.109]$} & {$[2.393]$} & {$[1.755]$} \\
maximin & 0.669 & 0.509 & 0.683 & 0.646 & 0.483 & 0.700 & 0.601 & 0.502 & 0.749 \\
& {$[0.924]$} & {$[0.491]$} & {$[0.491]$} & {$[0.791]$} & {$[0.425]$} & {$[0.488]$} & {$[0.627]$} & {$[0.426]$} & {$[0.517]$} \\
heuristic & -0.013 & 0.749 & 0.932 & 0.298 & 0.844 & 0.942 & 0.627 & 0.887 & 0.954 \\
& {$[2.545]$} & {$[1.603]$} & {$[1.152]$} & {$[2.167]$} & {$[1.389]$} & {$[1.098]$} & {$[1.763]$} & {$[1.269]$} & {$[1.079]$} \\
\hline \hline
\end{tabular}

Table 4 Mean of profit distribution for low profit margin.

approach typically gives an order quantity that is closer to the true optimal one, while the maximax (resp., maximin) approach, due to its optimism (resp., pessimism) tends to overorder (resp., underorder); see evidences in Figure 1. These observations motivate us a heuristic that averages the optimal maximax and maximin decisions, which turns out to perform very well (especially with a large training sample size, e.g., $N=50$ ) and can even be outstanding when the profit margin is medium; see Table 3.

As expected, the Hurwicz approach with a cross validated level of optimism outperforms its special cases of maximax and maximin approaches. Its performance appears to be only slightly inferior to the minimax approach when the training sample is 50. However, the level of optimism we have calibrated varies according to the combination of profit margin, demand and yield dependency, and training sample size. In the convention of decision theory, the level of optimism should be articulated by decision-makers (which may not be an easy task) and may not be subject to change.

Figure 2 shows the percentage reduction in profit uncertainty due to the minimax regret criterion:

$$
\frac{\gamma^{\star}}{\Gamma^{\star}}=\frac{\max _{\mathbb{P} \in \mathcal{F}_{\infty}(\theta)} \max _{y}\left\{\mathbb{E}_{\mathbb{P}}\left[\Pi(y, \tilde{u}, \tilde{v})-\Pi\left(x_{\mathrm{R}}^{\star}, \tilde{u}, \tilde{v}\right)\right]\right\}}{\max _{x} \max _{\mathbb{P} \in \mathcal{F}_{\infty}(\theta)} \mathbb{E}_{\mathbb{P}}[\Pi(x, \tilde{u}, \tilde{v})]-\max _{x} \min _{\mathbb{P} \in \mathcal{F}_{\infty}(\theta)} \mathbb{E}_{\mathbb{P}}[\Pi(x, \tilde{u}, \tilde{v})]} .
$$

The percentage reduction is typically much less than one, revealing a strict advantage of the minimax regret criterion over the most optimistic (resp., pessimistic) perspective of the maximax (resp., maximin) decision criterion in predicting the profit uncertainty. The percentage reduction becomes less significant when the profit margin is higher, or the training sample size is larger.

\section{Effect of training sample size}

The difference between the minimax regret approach and the maximax, maximin, Hurwicz, and heuristic ones is more significant when the training sample size is smaller. This is intuitive because 

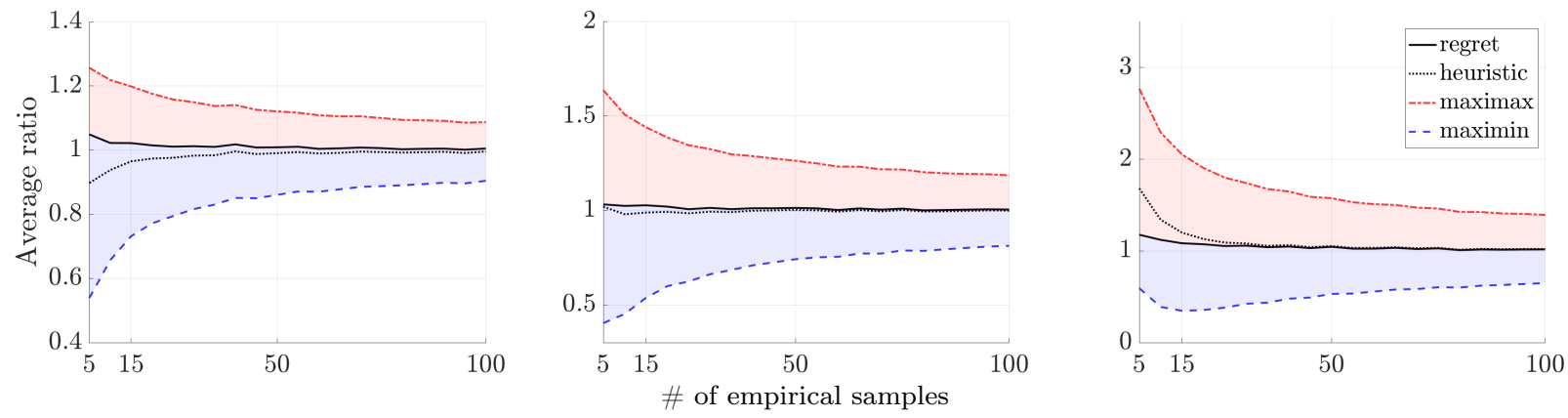

Figure 1 Average ratio to the true optimal order quantity among 1000 random trials. Here the ratio is defined as, for example, $x_{\mathrm{R}}^{\star} / x_{\mathrm{o}}^{\star}$. Uncorrelated demand and yield under high profit margin $(c=3$, left $)$, median profit ( $c=6$, middle), and low profit margin $(c=9$, right).
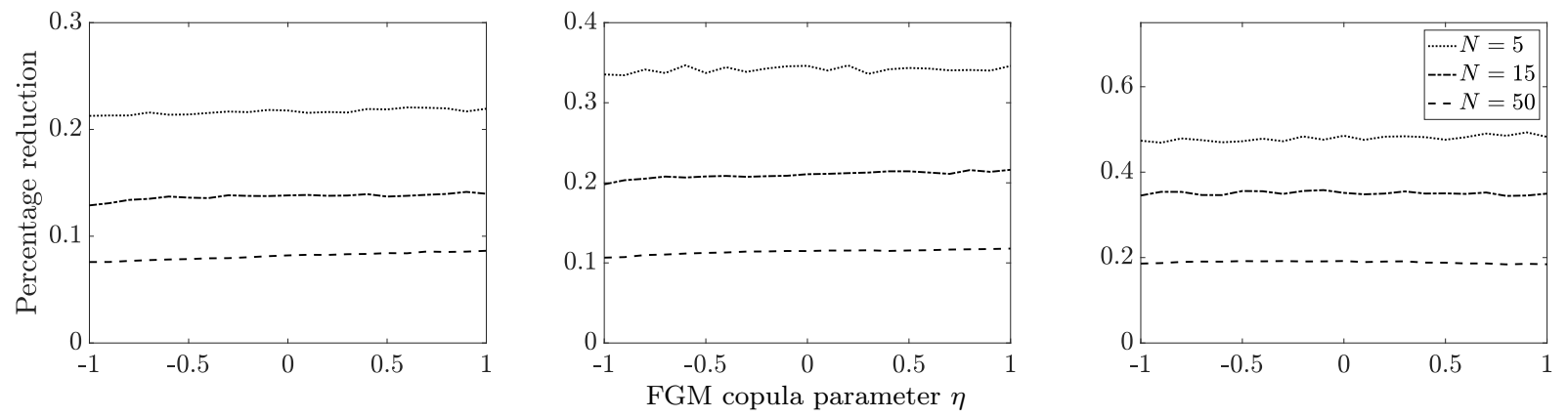

Figure 2 Percentage reduction in profit uncertainty due to the minimax regret crierion (average across 1000 random trials): high profit margin (left), median profit margin (middle), and low profit margin (right).

the Wasserstein ball shrinks and becomes closer to the true distribution as the sample size increases. Thus, all the approaches herein, being data-driven, improve with more training samples and move closer to the true optimum; see evidences in Figure 3. For this particular experiment where the underlying true distribution has uniform marginals and is characterized through the FGM copula, the empirical distribution consisting of $N=50$ samples could provide a good approximation of the true distribution; see the performance of different approaches when $N=50$ in Figure 3 and Tables. However $N=50$ samples may not be sufficient for other underlying true distribution: for example, in Section 6.3 we observe that 50 training samples is not yet sufficient for a low-profit-margin newsvendor model with a beta distribution, even when there is only demand randomness.

\section{Effect of profit margin}

Fixing the demand and yield dependency as well as the training sample size, when the profit margin is higher, the minimax approach yields an order quantity closer to the true optimal order quantity and thus has a smaller gap to the true optimality. We also notice that the maximax approach outperforms the maximin approach when the profit margin is high, whereas the latter performs 

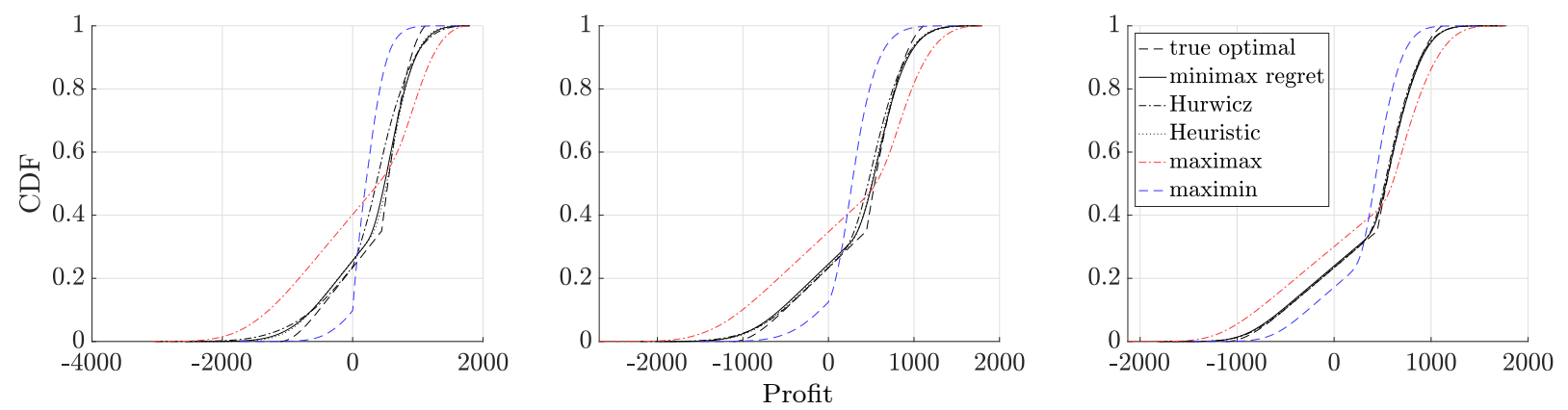

Figure 3 Profit CDF under negative correlated demand and yield of a small sample size $(N=5$, left), a median sample size $(N=15$, middle), and a large sample size $(N=50$, right). Profit margin is median, $c=6$.
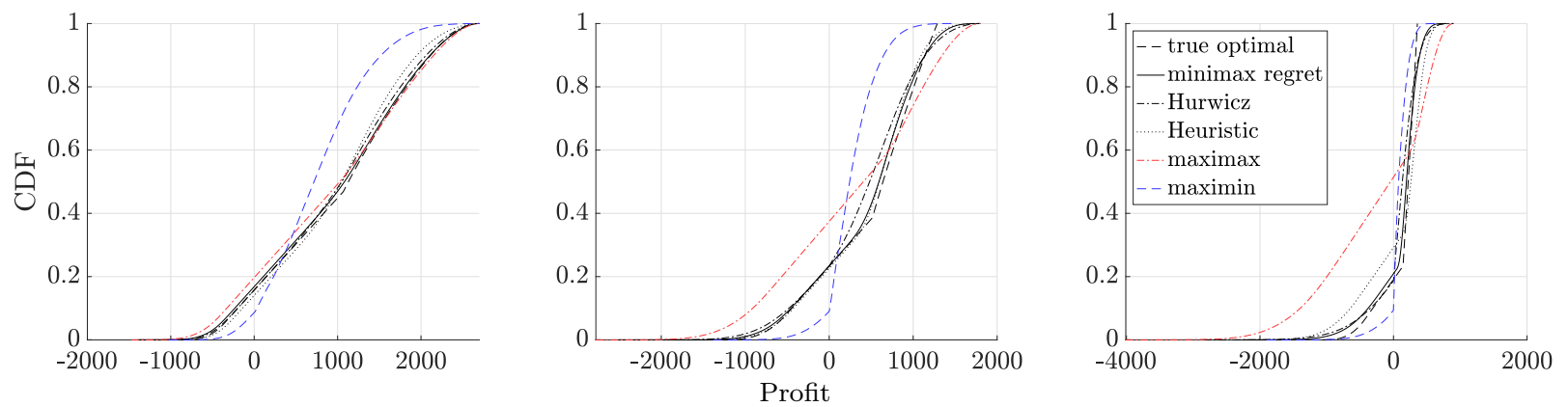

Figure 4 Profit CDF under positive correlated demand and yield with high profit margin $(c=3$, left), median profit margin ( $c=6$, middle), and low profit margin ( $c=9$, right). Training sample size is $N=5$.

better when the profit margin is low; see the trend in Figure 4. The intuition is that the aggressive maximax approach can exploit the opportunity of overordering for high profit margin. In contrast, the maximin approach is more beneficial by playing conservatively when the profit margin is low.

\subsection{Comparisons of Distance-Based and Moment-Based Models with Only Demand Randomness}

In this experiment we focus on the minimax regret newsvendor model with only demand randomness to compare the distance-based DRO approach with the moment-based one, whose optimal order quantity has been unified in Perakis and Roels (2008). In particular, we assume that the underlying true demand distribution is $(i)$ a uniform one in $[\underline{v}, \bar{v}]=[0,300],(i i)$ an exponential one with mean $\nu=150$, and (iii) a normal one with mean $\nu=150$ and standard deviation $\sigma=50$. For case $(i)$, we compare our distance-based $(\mathrm{DB})$ minimax regret model with a type- $\infty$ Wasserstein ball $\mathcal{F}_{\infty}(\theta)$ against a moment-based (MB) minimax regret model with an ambiguity set

$$
\mathcal{F}_{\mathrm{U}}=\left\{\mathbb{P}\left(\mathbb{R}_{+}\right) \mid \mathbb{P}[\tilde{v} \in[\underline{v}, \bar{v}]]=1, \mathbb{E}_{\mathbb{P}}[1]=1\right\},
$$


whose members share the same support information. For cases (ii) and (iii), we compare, respectively, against the MB model with the support-mean ambiguity set

$$
\mathcal{F}_{\mathrm{E}}=\left\{\mathbb{P}\left(\mathbb{R}_{+}\right) \mid \mathbb{P}[\tilde{v} \in[0, \infty)]=1, \mathbb{E}_{\mathbb{P}}[1]=1, \mathbb{E}_{\mathbb{P}}[\tilde{v}]=\nu\right\}
$$

and the support-mean-variance ambiguity set

$$
\mathcal{F}_{\mathrm{N}}=\left\{\mathbb{P}\left(\mathbb{R}_{+}\right) \mid \mathbb{P}[\tilde{v} \in[0, \infty)]=1, \mathbb{E}_{\mathbb{P}}[1]=1, \mathbb{E}_{\mathbb{P}}[\tilde{v}]=\nu, \mathbb{E}_{\mathbb{P}}\left[\tilde{v}^{2}\right]=\nu^{2}+\sigma^{2}\right\}
$$

Note that the underlying true uniform (resp., exponential) demand distribution is the entropymaximizing distribution in $\mathcal{F}_{\mathrm{U}}$ (resp., $\mathcal{F}_{\mathrm{E}}$ ) and the normal distribution is the entropy-maximizing distribution in a mean-variance variant of $\mathcal{F}_{\mathrm{N}}$ without considering the nonnegative support; see e.g. Perakis and Roels 2008. On the one hand, while the optimal order quantity in the MB model admits a closed-form expression in cases $(i)$ and $(i i)$ and can be efficiently determined with a gradient search in case ( iii), the optimal decision in our DB model, as we have mentioned, can be determined in less than a second via a golden section search. On the other hand, while our DB approach still works in the case of both demand and yield randomness, it is unclear how to extend the MB approach to consider both demand and yield randomness (see Section 3.1 for an explanation).

The procedure and parameters remain the same as in Section 6.1. The only difference is that here we consider two types of inputs for the moment-based ambiguity sets, resulting in two different models of implementing the moment-based approach. The first model, called MB-T ${ }^{6}$ model, takes directly parameters from the true underlying distribution and thus is independent of training samples; while the second one, called $\mathrm{MB}-\mathrm{E}^{7}$ model, estimates the inputs from random training samples: e.g., lower and upper bounds $\underline{v}$ and $\bar{v}$ of demand are estimated respectively by the smallest and largest sample values, and the mean $\nu$ (standard deviation $\sigma$ ) is estimated by sample mean (sample standard deviation). Averages results among 1000 random trials are reported in Tables 5 to 7 (see also the corresponding profit CDFs in Appendix E).

For case $(i)$ of uniform demand distribution in Table 5 , the performance of MB-E model improves and approaches to that of the MB-T model (indeed, the true optimum ${ }^{8}$ ) as the moment estimates become more accurate with an increasing number of training samples.Thus, in this very special case, the DB model shall not be expected to outperform the MB models, and we note that the DB model may not perform well when the profit margin is low. Nevertheless, the performance gap of our DB model remains within $5 \%$ for 6 out of 9 (10\% for 7 out of 9$)$ considered scenarios. The difference

\footnotetext{
${ }^{6}$ The ' $\mathrm{T}$ ' stands for true parameters.

${ }^{7}$ The 'E' stands for estimated parameters.

${ }^{8}$ In case $(i)$, the optimal order quantity of the MB-T model is known to coincide with the true optimal order quantity; see e.g., Perakis and Roels 2008.
} 


\begin{tabular}{cccccccccc}
\hline \hline$(c, N)$ & $(3,5)$ & $(3,15)$ & $(3,50)$ & $(6,5)$ & $(6,15)$ & $(6,50)$ & $(9,5)$ & $(9,15)$ & $(9,50)$ \\
\hline \multirow{2}{*}{ DB } & 0.964 & 0.985 & 0.995 & 0.919 & 0.953 & 0.983 & 0.719 & 0.867 & 0.952 \\
& {$[0.769]$} & {$[0.977]$} & {$[0.993]$} & {$[1.030]$} & {$[1.031]$} & {$[1.011]$} & {$[1.163]$} & {$[1.219]$} & {$[1.096]$} \\
MB-E & 0.966 & 0.994 & 0.999 & 0.955 & 0.992 & 0.999 & 0.667 & 0.950 & 0.995 \\
& {$[0.909]$} & {$[0.963]$} & {$[0.990]$} & {$[1.017]$} & {$[1.001]$} & {$[0.999]$} & {$[1.662]$} & {$[1.210]$} & {$[1.059]$} \\
MB-T & 1.000 & 1.000 & 1.000 & 1.000 & 1.000 & 1.000 & 1.000 & 1.000 & 1.000 \\
& {$[1.000]$} & {$[1.000]$} & {$[1.000]$} & {$[1.000]$} & {$[1.000]$} & {$[1.000]$} & {$[1.000]$} & {$[1.000]$} & {$[1.000]$} \\
\hline \hline
\end{tabular}

Table 5 Mean and standard deviation (in brackets) of profit distribution with uniform demand distribution.

\begin{tabular}{cccccccccc}
\hline \hline$(c, N)$ & $(3,5)$ & $(3,15)$ & $(3,50)$ & $(6,5)$ & $(6,15)$ & $(6,50)$ & $(9,5)$ & $(9,15)$ & $(9,50)$ \\
\hline DB & 0.894 & 0.951 & 0.983 & 0.791 & 0.915 & 0.973 & 0.403 & 0.823 & 0.946 \\
& {$[1.079]$} & {$[1.037]$} & {$[1.015]$} & {$[1.377]$} & {$[1.113]$} & {$[1.053]$} & {$[2.114]$} & {$[1.426]$} & {$[1.096]$} \\
MB-E & 0.775 & 0.820 & 0.836 & 0.846 & 0.898 & 0.926 & 0.744 & 0.901 & 0.953 \\
& {$[0.590]$} & {$[0.550]$} & {$[0.537]$} & {$[0.775]$} & {$[0.695]$} & {$[0.683]$} & {$[1.468]$} & {$[1.303]$} & {$[1.059]$} \\
MB-T & 0.843 & 0.843 & 0.843 & 0.935 & 0.935 & 0.935 & 0.978 & 0.978 & 0.978 \\
& {$[0.529]$} & {$[0.529]$} & {$[0.529]$} & {$[0.671]$} & {$[0.671]$} & {$[0.671]$} & {$[1.220]$} & {$[1.220]$} & {$[1.220]$} \\
\hline \hline
\end{tabular}

Table 6 Mean and standard deviation (in brackets) of profit distribution with exponential demand distribution.

\begin{tabular}{cccccccccc}
\hline \hline$(c, N)$ & $(3,5)$ & $(3,15)$ & $(3,50)$ & $(6,5)$ & $(6,15)$ & $(6,50)$ & $(9,5)$ & $(9,15)$ & $(9,50)$ \\
\hline DB & 0.980 & 0.993 & 0.998 & 0.964 & 0.987 & 0.995 & 0.913 & 0.968 & 0.990 \\
& {$[1.079]$} & {$[1.031]$} & {$[1.005]$} & {$[1.020]$} & {$[1.007]$} & {$[1.006]$} & {$[0.873]$} & {$[0.950]$} & {$[0.980]$} \\
MB-E & 0.975 & 0.989 & 0.993 & 0.964 & 0.987 & 0.995 & 0.884 & 0.953 & 0.978 \\
& {$[0.913]$} & {$[0.920]$} & {$[0.915]$} & {$[1.019]$} & {$[1.007]$} & {$[1.006]$} & {$[1.329]$} & {$[1.218]$} & {$[1.181]$} \\
MB-T & 0.996 & 0.996 & 0.996 & 1.000 & 1.000 & 1.000 & 0.986 & 0.986 & 0.986 \\
& {$[0.921]$} & {$[0.921]$} & {$[0.921]$} & {$[1.000]$} & {$[1.000]$} & {$[1.000]$} & {$[1.172]$} & {$[1.172]$} & {$[1.172]$} \\
\hline \hline
\end{tabular}

Table 7 Mean and standard deviation (in brackets) of profit distribution with normal demand distribution.

would be even smaller when the sample size $N$ is beyond 50 . It is worth noting that the MB-E model is vulnerable to training samples: for example, when the training sample size is small, it may mislead to an order quantity that largely differs from that of the MB-T model. This cautions the use of MB-E model in many practical or data-driven situations where true parameters of the MB model are not available.

For case $(i i)$ of exponential demand distribution in Table 6 where even the optimal order quantity of the MB-T model does not coincide with the true optimal order quantity, the inherent conserva- 
tiveness of MB models may not be fully reduced with the availability of more empirical samples (see e.g. high and median profit margins). In contrast, our DB model yields an optimal quantity that performs reasonably well and with more training samples, it may start to outperform MB models (e.g., when profit margin is high or medium and the sample size $N=50$ ). Especially, if the profit margin is high, our DB model also appears to be good even with small sizes of training samples such as $N=5$ or $N=15$.

The case of normal demand distribution in Table 7 demonstrates situations in between cases $(i)$ and $(i i)$. When the profit margin is medium, the MB-T model yields an order quantity that happens to coincide with the true optimal order quantity, and thus the DB model shall not be expected to outperform the MB models. Nevertheless, the performance gap of DB model remains within $0.5 \%$ (4\%) for a large sample size $N=50$ (for a small sample size $N=5$ ). On the other hand, when the profit margin is high (low), although the MB-T model yields an order quantity that is not less than (more than) the true optimal one by $10 \%$, even with the availability of more training samples its inherent conservativeness may not be fully reduced, while the DB model consistently improves and is able to outperform the MB-T model; see $(c, N)=(3,50)$ and $(c, N)=(9,50)$. We remark that the MB models based on the support-mean-variance ambiguity set $\mathcal{F}_{\mathrm{N}}$ does not always yield an order quantity that happens to coincide with or to be close to the true optimal one; see Section 6.3.

\subsection{Test under Non-Stationary Distribution with Only Demand Randomness}

We focus on the minimax regret model with only demand randomness to conduct a test on its performance under non-stationary distribution. We note that such a setting can mimic practical situations such as the introduction of a new product, where the managers have data of similar products to predict the new product's demand. In particular, we compare our DB model with the type- $\infty$ Wasserstein ball $\mathcal{F}_{\infty}(\theta)$ against MB models with ambiguity sets $\mathcal{F}_{\mathrm{U}}, \mathcal{F}_{\mathrm{E}}$, and $\mathcal{F}_{\mathrm{N}}$. As before, we consider MB-T and MB-E models that account for true and estimated parameters, respectively.

We assume that the underlying true demand distribution is $\underline{v}+(\bar{v}-\underline{v}) \tilde{v}$, where $\underline{v}=0, \bar{v}=300$, and $\tilde{v} \sim \mathbb{B}(\alpha, \beta)$ follows a beta distribution whose parameters $(\alpha, \beta)$ vary when training and testing samples are generated. In particular, for each trial among the 1000 replications, we randomly generate a number $N=50$ of training samples from a beta demand distribution with $(\alpha, \beta)=(0.5,0.5)$ and another set of $10^{7}$ testing samples with varying distributional parameters that may differ from $(0.5,0.5)$. Other parameters remain the same as in Sections 6.1 and 6.2. Averages results among 1000 random trials are reported in Tables 8 and 9 for high and low profit margins, respectively.

We first compare among MB models with ambiguity sets $\mathcal{F}_{\mathrm{U}}, \mathcal{F}_{\mathrm{E}}$ and $\mathcal{F}_{\mathrm{N}}$. When the profit margin is high, the ones with $\mathcal{F}_{\mathrm{U}}$ and $\mathcal{F}_{\mathrm{N}}$ dominates that with $\mathcal{F}_{\mathrm{E}}$, whereas the one with $\mathcal{F}_{\mathrm{E}}{ }^{9}$ becomes

${ }^{9}$ When the profit margin is low, the MB model with $\mathcal{F}_{\mathrm{E}}$ performs very well. This is because that it actually hedges against a worst-case distribution whose $(p-c) / c$-quantile is quite close to the true optimal order quantity of those testing beta distributions; see theorem 2 in Perakis and Roels (2008). 


\begin{tabular}{cccccccccc}
\hline \hline $10 \times(\alpha, \beta)$ & $(5,5)$ & $(5,4.8)$ & $(5,4.9)$ & $(5,5.1)$ & $(5,5.2)$ & $(4.8,5)$ & $(4.9,5)$ & $(5.1,5)$ & $(5.2,5)$ \\
\hline $\mathrm{DB}$ & 0.994 & 0.993 & 0.993 & 0.994 & 0.994 & 0.994 & 0.994 & 0.994 & 0.993 \\
& {$[0.983]$} & {$[0.971]$} & {$[0.977]$} & {$[0.990]$} & {$[0.996]$} & {$[0.990]$} & {$[0.987]$} & {$[0.980]$} & {$[0.977]$} \\
$\mathrm{MB}-\mathrm{E}: \mathcal{F}_{\mathrm{U}}$ & 0.983 & 0.978 & 0.980 & 0.985 & 0.987 & 0.985 & 0.984 & 0.981 & 0.980 \\
& {$[0.895]$} & {$[0.882]$} & {$[0.889]$} & {$[0.902]$} & {$[0.909]$} & {$[0.903]$} & {$[0.899]$} & {$[0.892]$} & {$[0.888]$} \\
$\mathrm{MB}-\mathrm{T}: \mathcal{F}_{\mathrm{U}}$ & 0.983 & 0.978 & 0.981 & 0.985 & 0.987 & 0.985 & 0.984 & 0.982 & 0.981 \\
& {$[0.896]$} & {$[0.883]$} & {$[0.890]$} & {$[0.903]$} & {$[0.910]$} & {$[0.904]$} & {$[0.900]$} & {$[0.893]$} & {$[0.889]$} \\
$\mathrm{MB}-\mathrm{E}: \mathcal{F}_{\mathrm{E}}$ & 0.685 & 0.672 & 0.678 & 0.690 & 0.696 & 0.691 & 0.687 & 0.680 & 0.677 \\
& {$[0.441]$} & {$[0.432]$} & {$[0.437]$} & {$[0.446]$} & {$[0.450]$} & {$[0.448]$} & {$[0.445]$} & {$[0.438]$} & {$[0.434]$} \\
$\mathrm{MB}-\mathrm{T}: \mathcal{F}_{\mathrm{E}}$ & 0.684 & 0.672 & 0.678 & 0.690 & 0.696 & 0.692 & 0.688 & 0.681 & 0.677 \\
& {$[0.435]$} & {$[0.426]$} & {$[0.431]$} & {$[0.440]$} & {$[0.444]$} & {$[0.442]$} & {$[0.439]$} & {$[0.431]$} & {$[0.428]$} \\
$\mathrm{MB}-\mathrm{E}: \mathcal{F}_{\mathrm{N}}$ & 0.940 & 0.932 & 0.936 & 0.944 & 0.947 & 0.944 & 0.942 & 0.938 & 0.936 \\
& {$[0.796]$} & {$[0.783]$} & {$[0.790]$} & {$[0.803]$} & {$[0.809]$} & {$[0.804]$} & {$[0.800]$} & {$[0.792]$} & {$[0.789]$} \\
$\mathrm{MB}-\mathrm{T}: \mathcal{F}_{\mathrm{N}}$ & 0.943 & 0.935 & 0.939 & 0.947 & 0.950 & 0.947 & 0.945 & 0.941 & 0.939 \\
& {$[0.793]$} & {$[0.780]$} & {$[0.787]$} & {$[0.800]$} & {$[0.806]$} & {$[0.801]$} & {$[0.797]$} & {$[0.789]$} & {$[0.786]$} \\
\hline \hline
\end{tabular}

Table 8 Mean and standard deviation (in brackets) of profit distribution under varying training and testing beta distributions. High profit margin.

\begin{tabular}{ccccccccccc}
\hline \hline $10 \times(\alpha, \beta)$ & $(5,5)$ & $(5,4.8)$ & $(5,4.9)$ & $(5,5.1)$ & $(5,5.2)$ & $(4.8,5)$ & $(4.9,5)$ & $(5.1,5)$ & $(5.2,5)$ \\
\hline $\mathrm{DB}$ & 0.865 & 0.881 & 0.874 & 0.856 & 0.843 & 0.816 & 0.844 & 0.879 & 0.889 \\
& {$[1.263]$} & {$[1.188]$} & {$[1.228]$} & {$[1.299]$} & {$[1.336]$} & {$[1.416]$} & {$[1.335]$} & {$[1.199]$} & {$[1.136]$} \\
$\mathrm{MB}-\mathrm{E}: \mathcal{F}_{\mathrm{U}}$ & 0.635 & 0.716 & 0.678 & 0.594 & 0.546 & 0.450 & 0.549 & 0.704 & 0.764 \\
& {$[1.882]$} & {$[1.772]$} & {$[1.832]$} & {$[1.936]$} & {$[1.990]$} & {$[2.106]$} & {$[1.987]$} & {$[1.789]$} & {$[1.699]$} \\
$\mathrm{MB}-\mathrm{T}: \mathcal{F}_{\mathrm{U}}$ & 0.642 & 0.721 & 0.685 & 0.601 & 0.554 & 0.459 & 0.556 & 0.710 & 0.770 \\
& {$[1.874]$} & {$[1.764]$} & {$[1.823]$} & {$[1.927]$} & {$[1.981]$} & {$[2.096]$} & {$[1.978]$} & {$[1.781]$} & {$[1.699]$} \\
$\mathrm{MB}-\mathrm{E}: \mathcal{F}_{\mathrm{E}}$ & 0.976 & 0.987 & 0.982 & 0.970 & 0.961 & 0.940 & 0.961 & 0.986 & 0.991 \\
& {$[1.176]$} & {$[1.106]$} & {$[1.144]$} & {$[1.211]$} & {$[1.245]$} & {$[1.322]$} & {$[1.244]$} & {$[1.115]$} & {$[1.056]$} \\
$\mathrm{MB}-\mathrm{T}: \mathcal{F}_{\mathrm{E}}$ & 0.986 & 0.995 & 0.991 & 0.979 & 0.971 & 0.951 & 0.971 & 0.994 & 0.999 \\
& {$[1.166]$} & {$[1.096]$} & {$[1.133]$} & {$[1.200]$} & {$[1.234]$} & {$[1.311]$} & {$[1.233]$} & {$[1.105]$} & {$[1.046]$} \\
$\mathrm{MB}-\mathrm{E}: \mathcal{F}_{\mathrm{N}}$ & -0.079 & 0.094 & 0.012 & -0.165 & -0.264 & -0.452 & -0.254 & 0.066 & 0.198 \\
& {$[2.568]$} & {$[2.420]$} & {$[2.500]$} & {$[2.640]$} & {$[2.711]$} & {$[2.862]$} & {$[2.706]$} & {$[2.445]$} & {$[2.325]$} \\
$\mathrm{MB}-\mathrm{T}: \mathcal{F}_{\mathrm{N}}$ & -0.001 & 0.167 & 0.087 & -0.084 & -0.179 & -0.363 & -0.172 & 0.139 & 0.266 \\
& {$[2.515]$} & {$[2.371]$} & {$[2.449]$} & {$[2.586]$} & {$[2.657]$} & {$[2.805]$} & {$[2.651]$} & {$[2.395]$} & {$[2.277]$} \\
\hline \hline
\end{tabular}

Table 9 Mean and standard deviation (in brackets) of profit distribution under varying training and testing beta distributions. Low profit margin. 
dominating when profit margin is low. Actually, the dominated one(s) among them could be inferior, regardless of whether the parameters are estimated or taken as the truth. This shows the need of specifying a "proper" ambiguity set in the MB model. The contrasting superior and inferior performances of MB models with $\mathcal{F}_{\mathrm{N}}$ when the profit margin is high and low raise the same caution.

We now turn to our purely data-driven DB model. It remains competitive in both cases of high and low profit margins: even if the testing distribution is different from the training distribution, the performance gap of our DB model is less than $1 \%$ for all eight scenarios ( $15 \%$ for 6 out of 8 scenarios) when the profit margin is high (low).

Of course, observations above maybe an artifact of the beta distribution. This motivates us to repeat the same experiment in a setting where the true normal demand has mean $\mu$ and standard deviation $\sigma$. For each trial we randomly generate training samples from this normal distribution and another set of normally distributed testing samples with varying mean and standard deviation that may differ from $(150,50)$. Other parameters and procedures remain the same as before. Results among 1000 random trials are reported in Tables 10 and 11. In this test, the MB models (especially, with the support-mean-variance ambiguity set) could perform well when tested under non-stationary distribution. Meanwhile, our DB model is also competitive: its performance gap is less than $1 \%$ (5\%) for 6 out of 8 scenarios when the profit margin is high (low). In 18 out of 36 situation in this section, it performs the best among all alternatives.

\section{Conclusion}

As a discrete distribution can always be converted into one that is uniformly supported on a finite set of (perhaps identical) points, our model can be straightforwardly generalized to arbitrarily centered distributions that are discrete. Our data-driven minimax regret approach, which is based on the type- $\infty$ Wasserstein distance, remains open to more complex inventory models. We believe that joint inventory and pricing might be of particular interest; see e.g. Caldentey et al. (2017), Chen et al. (2017), Kouvelis et al. (2018) to scholars examining pricing strategy under minimax regret or pricing strategy facing yield randomness. Another interesting direction is to apply the distancebased minimax regret approach to multi-item newsvendor models (see e.g. Hanasusanto et al. 2015, Natarajan and Teo 2017, Natarajan et al. 2018 for existing works that consider newsvendor models with multiple items under distributional ambiguity).

\section{Acknowledgments}

The authors are grateful to the editors and two anonymous reviewers for constructive and valuable comments that led to substantial improvements of the paper. The research of Zhi Chen is funded by the Early Career Scheme from the Hong Kong Research Grants Council (project number: 9048191). 


\begin{tabular}{cccccccccc}
\hline \hline$(\nu, \sigma) / 10$ & $(15,5)$ & $(15,3)$ & $(15,4)$ & $(15,6)$ & $(15,7)$ & $(13,5)$ & $(14,5)$ & $(16,5)$ & $(17,5)$ \\
\hline DB & 0.998 & 0.987 & 0.995 & 0.997 & 0.994 & 0.982 & 0.993 & 0.996 & 0.988 \\
& {$[1.009]$} & {$[1.129]$} & {$[1.061]$} & {$[0.971]$} & {$[0.941]$} & {$[1.117]$} & {$[1.068]$} & {$[0.942]$} & {$[0.867]$} \\
MB-E: $\mathcal{F}_{\mathrm{U}}$ & 0.979 & 0.949 & 0.967 & 0.987 & 0.991 & 0.940 & 0.963 & 0.988 & 0.991 \\
& {$[1.113]$} & {$[1.218]$} & {$[1.162]$} & {$[1.070]$} & {$[1.035]$} & {$[1.185]$} & {$[1.153]$} & {$[1.064]$} & {$[1.007]$} \\
MB-T: $\mathcal{F}_{\mathrm{U}}$ & 0.955 & 0.910 & 0.935 & 0.971 & 0.983 & 0.897 & 0.930 & 0.975 & 0.988 \\
& {$[1.190]$} & {$[1.256]$} & {$[1.229]$} & {$[1.149]$} & {$[1.112]$} & {$[1.231]$} & {$[1.214]$} & {$[1.159]$} & {$[1.118]$} \\
MB-E: $\mathcal{F}_{\mathrm{E}}$ & 0.804 & 0.804 & 0.807 & 0.798 & 0.789 & 0.880 & 0.843 & 0.766 & 0.727 \\
& {$[0.429]$} & {$[0.297]$} & {$[0.369]$} & {$[0.475]$} & {$[0.509]$} & {$[0.589]$} & {$[0.507]$} & {$[0.359]$} & {$[0.296]$} \\
MB-T: $\mathcal{F}_{\mathrm{E}}$ & 0.805 & 0.805 & 0.808 & 0.799 & 0.790 & 0.882 & 0.844 & 0.767 & 0.728 \\
& {$[0.421]$} & {$[0.252]$} & {$[0.352]$} & {$[0.470]$} & {$[0.507]$} & {$[0.585]$} & {$[0.501]$} & {$[0.348]$} & {$[0.281]$} \\
MB-E: $\mathcal{F}_{\mathrm{N}}$ & 0.994 & 0.997 & 0.997 & 0.988 & 0.982 & 0.995 & 0.998 & 0.983 & 0.967 \\
& {$[0.920]$} & {$[1.019]$} & {$[0.960]$} & {$[0.892]$} & {$[0.871]$} & {$[1.502]$} & {$[0.990]$} & {$[0.843]$} & {$[0.761]$} \\
MB-T: $\mathcal{F}_{\mathrm{N}}$ & 0.996 & 0.999 & 0.999 & 0.990 & 0.984 & 0.997 & 0.999 & 0.985 & 0.969 \\
& {$[0.921]$} & {$[1.024]$} & {$[0.962]$} & {$[0.892]$} & {$[0.871]$} & {$[1.504]$} & {$[0.992]$} & {$[0.843]$} & {$[0.759]$} \\
\hline \hline
\end{tabular}

Table 10 Mean and standard deviation (in brackets) of profit distribution under varying training and testing normal distributions. High profit margin.

\begin{tabular}{cccccccccc}
\hline \hline$(\nu, \sigma) / 10$ & $(15,5)$ & $(15,3)$ & $(15,4)$ & $(15,6)$ & $(15,7)$ & $(13,5)$ & $(14,5)$ & $(16,5)$ & $(17,5)$ \\
\hline DB & 0.990 & 0.953 & 0.980 & 0.986 & 0.968 & 0.917 & 0.976 & 0.975 & 0.942 \\
& {$[0.983]$} & {$[0.653]$} & {$[0.844]$} & {$[1.083]$} & {$[1.157]$} & {$[1.349]$} & {$[1.160]$} & {$[0.819]$} & {$[0.671]$} \\
MB-E: $\mathcal{F}_{\mathrm{U}}$ & 0.906 & 0.815 & 0.867 & 0.933 & 0.948 & 0.943 & 0.936 & 0.864 & 0.818 \\
& {$[0.729]$} & {$[0.525]$} & {$[0.609]$} & {$[0.839]$} & {$[0.931]$} & {$[1.031]$} & {$[0.871]$} & {$[0.607]$} & {$[0.505]$} \\
MB-T: $\mathcal{F}_{\mathrm{U}}$ & 0.800 & 0.668 & 0.737 & 0.853 & 0.896 & 0.922 & 0.862 & 0.740 & 0.684 \\
& {$[0.416]$} & {$[0.097]$} & {$[0.253]$} & {$[0.559]$} & {$[0.680]$} & {$[0.661]$} & {$[0.528]$} & {$[0.322]$} & {$[0.245]$} \\
MB-E: $\mathcal{F}_{\mathrm{E}}$ & 0.558 & 0.447 & 0.501 & 0.613 & 0.660 & 0.682 & 0.616 & 0.508 & 0.464 \\
& {$[0.214]$} & {$[0.057]$} & {$[0.105]$} & {$[0.337]$} & {$[0.454]$} & {$[0.369]$} & {$[0.283]$} & {$[0.160]$} & {$[0.119]$} \\
MB-T: $\mathcal{F}_{\mathrm{E}}$ & 0.559 & 0.447 & 0.501 & 0.614 & 0.661 & 0.683 & 0.617 & 0.508 & 0.465 \\
& {$[0.212]$} & {$[0.021]$} & {$[0.097]$} & {$[0.336]$} & {$[0.454]$} & {$[0.367]$} & {$[0.281]$} & {$[0.157]$} & {$[0.115]$} \\
MB-E: $\mathcal{F}_{\mathrm{N}}$ & 0.979 & 0.988 & 0.989 & 0.950 & 0.905 & 0.800 & 0.920 & 0.993 & 0.982 \\
& {$[1.185]$} & {$[0.925]$} & {$[1.081]$} & {$[1.257]$} & {$[1.309]$} & {$[1.566]$} & {$[1.372]$} & {$[1.005]$} & {$[0.838]$} \\
MB-T: $\mathcal{F}_{\mathrm{N}}$ & 0.986 & 0.997 & 0.999 & 0.960 & 0.916 & 0.815 & 0.932 & 1.000 & 0.988 \\
& {$[1.172]$} & {$[0.886]$} & {$[1.060]$} & {$[1.248]$} & {$[1.302]$} & {$[1.555]$} & {$[1.361]$} & {$[0.991]$} & {$[0.822]$} \\
\hline \hline
\end{tabular}

Table 11 Mean and standard deviation (in brackets) of profit distribution under varying training and testing normal distributions. Low profit margin. 


\section{References}

Ausubel, Lawrence, Raymond Deneckere. 1993. A generalized theorem of the maximum. Economic Theory 3(1) 99-107.

Ben-Tal, Aharon, Dick den Hertog, Anja De Waegenaere, Bertrand Melenberg, Gijs Rennen. 2013. Robust solutions of optimization problems affected by uncertain probabilities. Management Science 59(2) $341-357$.

Ben-Tal, Aharon, Arkadi Nemirovski. 2000. Robust solutions of linear programming problems contaminated with uncertain data. Mathematical Programming 88(3) 411-424.

Bertsimas, Dimitris, Iain Dunning. 2020. Relative robust and adaptive optimization. INFORMS Journal on Computing 32(2) 408-427.

Bertsimas, Dimitris, Shtern Shimrit, Sturt Bradley. 2018. A data-driven approach for multi-stage linear optimization. Available at Optimization Online.

Bertsimas, Dimitris, Melvyn Sim. 2004. The price of robustness. Operations Research 52(1) 35-53.

Bimpikis, Kostas, Douglas Fearing, Alireza Tahbaz-Salehi. 2018. Multisourcing and miscoordination in supply chain networks. Operations Research 66(4) 1023-1039.

Blanchet, Jose, Karthyek Murthy. 2019. Quantifying distributional model risk via optimal transport. Mathematics of Operations Research 44(2) 565-600.

Boyd, Stephen, Lieven Vandenberghe. 2004. Convex optimization. Cambridge University Press.

Caldentey, René, Ying Liu, Ilan Lobel. 2017. Intertemporal pricing under minimax regret. Operations Research 65(1) 104-129.

Chen, Zhi, Melvyn Sim, Peng Xiong. 2020. Robust stochastic optimization made easy with RSOME. Management Science 66(8) 3329-3339.

Chen, Zhi, Melvyn Sim, Huan Xu. 2019. Distributionally robust optimization with infinitely constrained ambiguity sets. Operations Research 67(5) 1328-1344.

Chen, Zhi-Long, Nicholas Hall, Hans Kellerer. 2017. Dynamic pricing to minimize maximum regret. Production and Operations Management 26(1) 47-63.

Cossette, Helene, Etienne Marceau, Fouad Marri. 2008. On the compound Poisson risk model with dependence based on a generalized Farlie-Gumbe-Morgenstern copula. Insurance: Mathematics and Economics 43(3) 444-455.

DeCroix, Gregory A. 2013. Inventory management for an assembly system subject to supply disruptions. Management Science 59(9) 2079-2092.

Delage, Erick, Yinyu Ye. 2010. Distributionally robust optimization under moment uncertainty with application to data-driven problems. Operations Research 58(3) 595-612. 
El Ghaoui, Laurent, Maksim Oks, Francois Oustry. 2003. Worst-case value-at-risk and robust portfolio optimization: a conic programming approach. Operations Research 51(4) 543-556.

Ellsberg, Daniel. 1961. Risk, ambiguity, and the savage axioms. The Quarterly Journal of Economics $\mathbf{7 5}$ 643-669.

Gallego, Guillermo. 1998. New bounds and heuristics for (Q, R) policies. Management Science 44(2) 219-233.

Gallego, Guillermo, Ilkyeong Moon. 1993. The distribution free newsboy problem: review and extensions. Journal of the Operational Research Society 44(8) 825-834.

Gallego, Guillermo, Jennifer Ryan, David Simchi-Levi. 2001. Minimax analysis for finite-horizon inventory models. IIE Transactions 33(10) 861-874.

Gao, Rui, Anton Kleywegt. 2016. Distributionally robust stochastic optimization with Wasserstein distance. arXiv preprint arXiv:1604.02199.

Gilboa, Itzhak, David Schmeidler. 1989. Maxmin expected utility with non-unique prior. Journal of Mathematical Economics 18(2) 141-153.

Givens, Clark, Rae Michael Shortt. 1984. A class of Wasserstein metrics for probability distributions. The Michigan Mathematical Journal 31(2) 231-240.

Goh, Joel, Melvyn Sim. 2010. Distributionally robust optimization and its tractable approximations. Operations Research 58(4-part-1) 902-917.

Gupta, Varun, Bo He, Suresh Sethi. 2015. Contingent sourcing under supply disruption and competition. International Journal of Production Research 53(10) 3006-3027.

Hanasusanto, Grani, Daniel Kuhn, Stein Wallace, Steve Zymler. 2015. Distributionally robust multi-item newsvendor problems with multimodal demand distributions. Mathematical Programming 152(1-2) $1-32$.

Hurwicz, Leonid. 1951. The generalized Bayes minimax principle: a criterion for decision making uncer ucertainty. Cowles Comm. Discuss. Paper Stat (355).

Inderfurth, Karl, Gudrun Kiesmüller. 2015. Exact and heuristic linear-inflation policies for an inventory model with random yield and arbitrary lead times. European Journal of Operational Research 245(1) $109-120$.

Jiang, Ruiwei, Yongpei Guan. 2018. Risk-averse two-stage stochastic program with distributional ambiguity. Operations Research 66(5) 1390-1405.

Käki, Anssi, Juuso Liesiö, Ahti Salo, Srinivas Talluri. 2015. Newsvendor decisions under supply uncertainty. International Journal of Production Research 53(5) 1544-1560.

Kouvelis, Panos, Jian Li. 2013. Offshore outsourcing, yield uncertainty, and contingency responses. Production and Operations Management 22(1) 164-177. 
Kouvelis, Panos, Guang Xiao, Nan Yang. 2018. On the properties of yield distributions in random yield problems: conditions, class of distributions and relevant applications. Production and Operations Management 27(7) 1291-1302.

Kouvelis, Panos, Gang Yu. 2013. Robust discrete optimization and its applications. Springer Science \& Business Media.

Levi, Retsef, Georgia Perakis, Joline Uichanco. 2015. The data-driven newsvendor problem: new bounds and insights. Operations Research 63(6) 1294-1306.

Levi, Retsef, Robin Roundy, David Shmoys. 2007. Provably near-optimal sampling-based policies for stochastic inventory control models. Mathematics of Operations Research 32(4) 821-839.

Li, Bowen, Ruiwei Jiang, Johanna Mathieu. 2019. Ambiguous risk constraints with moment and unimodality information. Mathematical Programming 173(1-2) 151-192.

Lim, Andrew, George Shanthikumar, ZJ Max Shen. 2006. Model uncertainty, robust optimization, and learning. Models, Methods, and Applications for Innovative Decision Making. INFORMS, 66-94.

Lin, Jun, Tsan Sheng Ng. 2011. Robust multi-market newsvendor models with interval demand data. European Journal of Operational Research 212(2) 361-373.

Luce, Duncan, Howard Raiffa. 1989. Games and decisions: introduction and critical survey. Courier Corporation.

Mohajerin Esfahani, Peyman, Daniel Kuhn. 2018. Data-driven distributionally robust optimization using the Wasserstein metric: performance guarantees and tractable reformulations. Mathematical Programming 171(1-2) 1-52.

Natarajan, Karthik, Melvyn Sim, Joline Uichanco. 2018. Asymmetry and ambiguity in newsvendor models. Management Science 64(7) 3146-3167.

Natarajan, Karthik, Chung-Piaw Teo. 2017. On reduced semidefinite programs for second order moment bounds with applications. Mathematical Programming 161(1-2) 487-518.

Perakis, Georgia, Guillaume Roels. 2008. Regret in the newsvendor model with partial information. Operations Research 56(1) 188-203.

Popescu, Ioana. 2005. A semidefinite programming approach to optimal-moment bounds for convex classes of distributions. Mathematics of Operations Research 30(3) 632-657.

Poursoltani, Mehran, Erick Delage. 2019. Adjustable robust optimization reformulations of two-stage worstcase regret minimization problems. Available at Optimization Online.

Savage, L. J. 1951. The theory of statistical decisions. Journal of the American Statistical Association 46(253) 55-67.

Scarf, Herbert. 1958. A min-max solution of an inventory problem. Studies in the mathematical theory of inventory and production 10(2) 201. 
Schweitzer, Maurice, Gérard Cachon. 2000. Decision bias in the newsvendor problem with a known demand distribution: experimental evidence. Management Science 46(3) 404-420.

Serfling, Robert J. 2009. Approximation theorems of mathematical statistics, vol. 162. John Wiley \& Sons.

Tan, Burcu, Qi Feng, Wen Chen. 2016. Dual sourcing under random supply capacities: the role of the slow supplier. Production and Operations Management 25(7) 1232-1244.

Tang, Sammi Yu, Haresh Gurnani, Diwakar Gupta. 2014. Managing disruptions in decentralized supply chains with endogenous supply process reliability. Production and Operations Management 23(7) 11981211.

Tang, Sammi Yu, Panos Kouvelis. 2011. Supplier diversification strategies in the presence of yield uncertainty and buyer competition. Manufacturing \& Service Operations Management 13(4) 439-451.

Tang, Sammi Yu, Panos Kouvelis. 2014. Pay-back-revenue-sharing contract in coordinating supply chains with random yield. Production and Operations Management 23(12) 2089-2102.

Tomlin, Brian, Yimin Wang. 2011. Operational strategies for managing supply chain disruption risk. The handbook of integrated risk management in global supply chains 79-101.

Trillos, Nicolás Garcia, Dejan Slepčev. 2015. On the rate of convergence of empirical measures in $\infty$ transportation distance. Canadian Journal of Mathematics 67(6) 1358-1383.

Vairaktarakis, George. 2000. Robust multi-item newsboy models with a budget constraint. International Journal of Production Economics 66(3) 213-226.

Wang, Zizhuo, Peter Glynn, Yinyu Ye. 2016. Likelihood robust optimization for data-driven problems. Computational Management Science 13(2) 241-261.

Wiesemann, Wolfram, Daniel Kuhn, Melvyn Sim. 2014. Distributionally robust convex optimization. Operations Research 62(6) 1358-1376.

Xie, Weijun. 2020. Tractable reformulations of two-stage distributionally robust linear programs over the type- $\infty$ Wasserstein ball. Operations Research Letters 48(4) 513-523.

Xie, Weijun, Shabbir Ahmed. 2018. On deterministic reformulations of distributionally robust joint chance constrained optimization problems. SIAM Journal on Optimization 28(2) 1151-1182.

Xie, Weijun, Jie Zhang, Shabbir Ahmed. 2021. Distributionally robust bottleneck combinatorial problems: uncertainty quantification and robust decision making. Mathematical Programming 1-44.

Yano, Candace, Hau Lee. 1995. Lot sizing with random yields: a review. Operations Research 43(2) 311-334.

Yue, Jinfeng, Bintong Chen, Min-Chiang Wang. 2006. Expected value of distribution information for the newsvendor problem. Operations Research 54(6) 1128-1136.

Zhu, Zhisu, Jiawei Zhang, Yinyu Ye. 2013. Newsvendor optimization with limited distribution information. Optimization Methods and Software 28(3) 640-667. 


\section{Appendix A. Proofs}

Proof of Lemma 1. Since $\Lambda(x, y, u, v)=(p-c)(y-x) u+p(u x-v)^{+}-p(u y-v)^{+}$, for any fixed $u \in[0,1]$ we have

$$
\max _{v \in \mathcal{V}} \Lambda(x, y, u, v)=(p-c)(y-x) u+\max _{v \in \mathcal{V}}\left\{p(u x-v)^{+}-p(u y-v)^{+}\right\}
$$

It is therefore sufficient to consider the function $p(u x-v)^{+}-p(u y-v)^{+}$. If $y \geq x$ then the term

$$
p(u x-v)^{+}-p(u y-v)^{+}= \begin{cases}p(u x-u y) & \text { if } v \leq u x \\ p(v-u y) & \text { if } u x \leq v \leq u y \\ 0 & \text { if } v \geq u y\end{cases}
$$

is nondecreasing in $v$; whereas if $y \leq x$ then the term

$$
p(u x-v)^{+}-p(u y-v)^{+}= \begin{cases}p(u x-u y) & \text { if } v \leq u y \\ p(u x-v) & \text { if } u y \leq v \leq u x \\ 0 & \text { if } v \geq u x\end{cases}
$$

is nonincreasing in $v$. As a consequence,

$$
\max _{v \in \mathcal{V}}\left\{p(u x-v)^{+}-p(u y-v)^{+}\right\}= \begin{cases}p(u x-\bar{v})^{+}-p(u y-\bar{v})^{+} & \text {if } y \geq x \\ p(u x-\underline{v})^{+}-p(u y-\underline{v})^{+} & \text {if } y \leq x\end{cases}
$$

The statement of the theorem now follows.

Proof of Lemma 2. Recall that $\mathcal{U}=[\underline{u}, \bar{u}]$. We first look at the case of $y \geq x$. Given the values of $x, y$, and $v$ along with condition $y \geq x$, six scenarios must be considered when simplifying the objective function $\Lambda(x, y, u, v)=(p-c)(y-x) u+p(u x-v)^{+}-p(u y-v)^{+}$.

1. Suppose $v / \underline{u} \geq v / \bar{u} \geq y \geq x$. Since $u x \leq v$ and $u y \leq v$ for all $u \in \mathcal{U}$, it follows that $\Lambda(x, y, u, v)=$ $(p-c)(y-x) u-$ which is increasing in $u$ and, with $u^{\star}=\bar{u}$, gives

$$
\max _{u \in \mathcal{U}} \Lambda(x, y, u, v)=(p-c)(y-x) \bar{u}=(p-c) \bar{u} y-(p-c) \bar{u} x
$$

2. Suppose $v / \underline{u} \geq y \geq v / \bar{u} \geq x$. If $\underline{u} \leq u \leq v / y$ then $\Lambda(x, y, u, v)=(p-c)(y-x) u$, which is increasing in $u$ and yields

$$
\max _{u \in \mathcal{U}, \underline{u} \leq u \leq v / y} \Lambda(x, y, u, v)=(p-c)(y-x) \frac{v}{y} .
$$

If $v / y \leq u \leq \bar{u}$ then $\Lambda(x, y, u, v)=(-p x-c(y-x)) u+p v$, which is decreasing in $u$ and gives

$$
\max _{u \in \mathcal{U}, v / y \leq u \leq \bar{u}} \Lambda(x, y, u, v)=(p-c)(y-x) \frac{v}{y} .
$$

So in this case, we always have $u^{\star}=v / y$ and

$$
\max _{u \in \mathcal{U}} \Lambda(x, y, u, v)=(p-c)(y-x) \frac{v}{y}=-(p-c) \frac{x v}{y}+(p-c) v .
$$


3. Suppose $v / \underline{u} \geq y \geq x \geq v / \bar{u}$. If $\underline{u} \leq u \leq v / y$ then $\Lambda(x, y, u, v)=(p-c)(y-x) u$, which is increasing in $u$ and gives

$$
\max _{u \in \mathcal{U}, \underline{u} \leq u \leq v / y} \Lambda(x, y, u, v)=(p-c)(y-x) \frac{v}{y} .
$$

If $v / y \leq u \leq v / x$ then $\Lambda(x, y, u, v)=(-p x-c(y-x)) u+p v$, which is decreasing in $u$ and gives

$$
\max _{u \in \mathcal{U}, v / y \leq u \leq v / x} \Lambda(x, y, u, v)=(p-c)(y-x) \frac{v}{y} .
$$

If $v / x \leq u \leq \bar{u}$ then $\Lambda(x, y, u, v)=-c(y-x) u$, which is decreasing in $u$ and gives

$$
\max _{u \in \mathcal{U}, v / x \leq u \leq \bar{u}} \Lambda(x, y, u, v)=-c(y-x) \frac{v}{x} .
$$

Since $(p-c)(y-x) \frac{v}{y} \geq 0$ whereas $-c(y-x) \frac{v}{x} \leq 0$, in this case, we have $u^{\star}=v / y$ and

$$
\max _{u \in \mathcal{U}} \Lambda(x, y, u, v)=(p-c)(y-x) \frac{v}{y}=-(p-c) \frac{x v}{y}+(p-c) v .
$$

4. Suppose $y \geq v / \underline{u} \geq v / \bar{u} \geq x$. Then we have $\Lambda(x, y, u, v)=(-p x-c(y-x)) u+p v$, which is decreasing in $u$ and, with $u^{\star}=\underline{u}$, gives

$$
\max _{u \in \mathcal{U}} \Lambda(x, y, u, v)=(-p x-c(y-x)) \underline{u}+p v=-c \underline{u} y-(p-c) \underline{u} x+p v .
$$

5. Suppose $y \geq v / \underline{u} \geq x \geq v / \bar{u}$. If $\underline{u} \leq u \leq \frac{v}{x}$ then $\Lambda(x, y, u, v)=(-p x-c(y-x)) u+p v$, which is decreasing in $u$ and yields

$$
\max _{u \in \mathcal{U}, \underline{u} \leq u \leq v / x} \Lambda(x, y, u, v)=(-p x-c(y-x)) \underline{u}+p v .
$$

If $v / x \leq u \leq \bar{u}$ then $\Lambda(x, y, u, v)=-c(y-x) u$, which is decreasing in $u$ (since $y \geq x$ ) and gives

$$
\max _{u \in \mathcal{U}, v / x \leq u \leq \bar{u}} \Lambda(x, y, u, v)=-c(y-x) \frac{v}{x} .
$$

The difference between $(-p x-c(y-x)) \underline{u}+p v$ and $-c(y-x) \frac{v}{x}$ is given by $(-p x-c(y-x)) \underline{u}+$ $p v+c(y-x) \frac{v}{x}=p x\left(\frac{v}{x}-\underline{u}\right)+c(y-x)\left(\frac{v}{x}-\underline{u}\right) \geq 0$. In this case, then, $u^{\star}=\underline{u}$ and

$$
\max _{u \in \mathcal{U}} \Lambda(x, y, u, v)=(-p x-c(y-x)) \underline{u}+p v=-c \underline{u} y-(p-c) \underline{u} x+p v .
$$

6. Suppose $y \geq x \geq v / \underline{u} \geq v / \bar{u}$. Then $\Lambda(x, y, u, v)=-c(y-x) u$, which is decreasing in $u$ and, with $u^{\star}=\underline{u}$, gives

$$
\max _{u \in \mathcal{U}} \Lambda(x, y, u, v)=-c(y-x) \underline{u}=-c \underline{u} y+c \underline{u} x .
$$

We next discuss the six scenarios for the case of $y \leq x$ in a similar manner.

1. Suppose $v / \underline{u} \geq v / \bar{u} \geq x \geq y$. Since $u x \leq v$ and $u y \leq v$ for all $u \in \mathcal{U}$, it follows that $\Lambda(x, y, u, v)=$ $(p-c)(y-x) u-$ which is increasing in $u$ and, with $u^{\star}=\underline{u}$, gives

$$
\max _{u \in \mathcal{U}} \Lambda(x, y, u, v)=(p-c)(y-x) \underline{u}=(p-c) \underline{u} y-(p-c) \underline{u} x .
$$


2. Suppose $v / \underline{u} \geq x \geq v / \bar{u} \geq y$. If $\underline{u} \leq u \leq v / x$ then $\Lambda(x, y, u, v)=(p-c)(y-x) u$, which is decreasing in $u$ and yields

$$
\max _{u \in \mathcal{U}, \underline{u} \leq u \leq v / x} \Lambda(x, y, u, v)=(p-c)(y-x) \underline{u} .
$$

If $v / x \leq u \leq \bar{u}$ then $\Lambda(x, y, u, v)=(c x+(p-c) y) u-p v$, which is increasing in $u$ and gives

$$
\max _{u \in \mathcal{U}, v / x \leq u \leq \bar{u}} \Lambda(x, y, u, v)=(c x+(p-c) y) \bar{u}-p v
$$

Taking the difference between $(p-c)(y-x) \underline{u}$ and $(c x+(p-c) y) \bar{u}-p v$, we observe that

$$
(p-c)(y-x) \underline{u}-(c x+(p-c) y) \bar{u}+p v=\underbrace{(p-c)(\underline{u}-\bar{u}) y}_{\leq 0}+\underbrace{c(\underline{u}-\bar{u}) x}_{\leq 0}+\underbrace{p(v-\underline{u} x)}_{\geq 0},
$$

the sign of which could be either nonpositive or nonnegative. Therefore, in this case we have

$$
\max _{u \in \mathcal{U}} \Lambda(x, y, u, v)=\max \{(p-c) \underline{u} y-(p-c) \underline{u} x,(p-c) \bar{u} y+c \bar{u} x-p v\}
$$

with $u^{\star}=\underline{u}$ if $(p-c) \underline{u} y-(p-c) \underline{u} x \geq(p-c) \bar{u} y+c \bar{u} x-p v$ or $u^{\star}=\bar{u}$ otherwise.

3. Suppose $v / \underline{u} \geq x \geq y \geq v / \bar{u}$. If $\underline{u} \leq u \leq v / x$ then $\Lambda(x, y, u, v)=(p-c)(y-x) u$, which is decreasing in $u$ and gives

$$
\max _{u \in \mathcal{U}, \underline{u} \leq u \leq v / x} \Lambda(x, y, u, v)=(p-c)(y-x) \underline{u} \leq 0 .
$$

If $v / x \leq u \leq v / y$ then $\Lambda(x, y, u, v)=(c x+(p-c) y) u+p v$, which is increasing in $u$ and from which it follows that

$$
\max _{u \in \mathcal{U}, v / x \leq u \leq v / y} \Lambda(x, y, u, v)=(c x+(p-c) y) \frac{v}{y}-p v .
$$

If $v / y \leq u \leq \bar{u}$ then $\Lambda(x, y, u, v)=c(x-y) u$, which is increasing in $u$ and gives

$$
\max _{u \in \mathcal{U}, v / y \leq u \leq \bar{u}} \Lambda(x, y, u, v)=c(x-y) \bar{u} \geq 0 .
$$

Note that it is sufficient to look at the difference between $c(x-y) \bar{u}$ and $(c x+(p-c) y) \frac{v}{y}-p v$, which amounts to $c(x-y) \bar{u}-(c x+(p-c) y) \frac{v}{y}+p v=c x\left(\bar{u}-\frac{v}{y}\right)+p v-(p-c) v=c x\left(\bar{u}-\frac{v}{y}\right)+c v \geq$ 0 . Therefore, in this case $u^{\star}=\bar{u}$ and

$$
\max _{u \in \mathcal{U}} \Lambda(x, y, u, v)=c(x-y) \bar{u}=-c \bar{u} y+c \bar{u} x .
$$

4. Suppose $x \geq v / \underline{u} \geq v / \bar{u} \geq y$. Then $\Lambda(x, y, u, v)=(c x+(p-c) y) u-p v$, which is increasing in $u$ and, with $u^{\star}=\bar{u}$, results

$$
\max _{u \in \mathcal{U}} \Lambda(x, y, u, v)=(c x+(p-c) y) \bar{u}-p v=(p-c) \bar{u} y+c \bar{u} x-p v
$$


5. Suppose $x \geq v / \underline{u} \geq y \geq v / \bar{u}$. If $\underline{u} \leq u \leq \frac{v}{y}$ then $\Lambda(x, y, u, v)=(c x+(p-c) y) u-p v$, which is increasing in $u$ and gives

$$
\max _{u \in \mathcal{U}, \underline{u} \leq u \leq v / y} \Lambda(x, y, u, v)=(c x+(p-c) y) \frac{v}{y}-p v .
$$

If $v / y \leq u \leq \bar{u}$ then $\Lambda(x, y, u, v)=c(x-y) u$, which is increasing in $u$ (since $y \geq x$ ) and yields

$$
\max _{u \in \mathcal{U}, v / x \leq u \leq \bar{u}} \Lambda(x, y, u, v)=c(x-y) \bar{u}
$$

Taking the difference between $c(x-y) \bar{u}$ and $(c x+(p-c) y) \frac{v}{y}-p v$, we obtain $c(x-y) \bar{u}-c x \frac{v}{y}+$ $c v=c x\left(\bar{u}-\frac{v}{y}\right)-c y\left(\bar{u}-\frac{v}{y}\right)=c(x-y)\left(\bar{u}-\frac{v}{y}\right) \geq 0$. So here we have $u^{\star}=\bar{u}$ and

$$
\max _{u \in \mathcal{U}} \Lambda(x, y, u, v)=c(x-y) \bar{u}=-c \bar{u} y+c \bar{u} x .
$$

6. Suppose $x \geq y \geq v / \underline{u} \geq v / \bar{u}$. Then $\Lambda(x, y, u, v)=c(x-y) u$, which is increasing in $u$ and, with $u^{\star}=\bar{u}$, yields

$$
\max _{u \in \mathcal{U}} \Lambda(x, y, u, v)=c(x-y) \bar{u}=-c \bar{u} y+c \bar{u} x .
$$

To conclude, we summarize the results for both cases- $y \geq x$ and $y \leq x$-in Table 1.

Proof of Theorem 1. The proof proceeds in three steps. We prove that, in the two regions beyond both ends of the interval $[\underline{x}, \bar{x}]$, the corresponding optimal decisions in the minimax regret newsvendor model (5) are $\underline{x}$ and $\bar{x}$; see Step 1 and Step 2, respectively. In Step 3, we derive upper and lower bounds on the auxiliary decision $y$. For ease of notation, we put

$$
\bar{\Omega}(x, y)=\frac{1}{N} \sum_{i \in[N]} \max _{u \in \mathcal{U}_{i}} \Lambda\left(x, y, u, \bar{v}_{i}\right) \quad \text { and } \quad \Omega(x, y)=\frac{1}{N} \sum_{i \in[N]} \max _{u \in \mathcal{U}_{i}} \Lambda\left(x, y, u, \underline{v}_{i}\right),
$$

which, with Lemma 1, allow us to rewrite the minimax regret newsvendor model (5) as

$$
\min _{x} \max \left\{\max _{y \geq x} \bar{\Omega}(x, y), \max _{y \leq x} \Omega(x, y)\right\} .
$$

Step 1. For any fixed $x \leq \underline{x}$, Table 1 shows that on the domain $y \leq x$, the function $\Omega(x, y)=$ $\frac{1}{N} \sum_{i \in[N]}\left((p-c) \underline{u}_{i} y-(p-c) \underline{u}_{i} x\right)$ is nondecreasing in $y$. Hence $\max _{y \leq x} \Omega(x, y)=0$ with the maximum achieved at $y=x$. Thus we obtain

$$
\min _{x \leq \underline{x}} \max \left\{\max _{y \geq x} \bar{\Omega}(x, y), \max _{y \leq x} \Omega(x, y)\right\}=\min _{x \leq \underline{x}} \max \left\{\max _{y \geq x} \bar{\Omega}(x, y), 0\right\}=\min _{x \leq \underline{x}} \max _{y \geq x} \bar{\Omega}(x, y)
$$

here on the interval $x \leq \underline{x}$, the objective function of the inner maximization problem

$$
\bar{\Omega}(x, y)=\frac{1}{N} \sum_{i \in[N]: y \leq \bar{v}_{i} / \bar{u}_{i}}\left((p-c) \bar{u}_{i} y-(p-c) \bar{u}_{i} x\right)+\frac{1}{N} \sum_{i \in[N]: y>\bar{v}_{i} / \bar{u}_{i}}\left(-(p-c) \frac{x \bar{v}_{i}}{y}+(p-c) \bar{v}_{i}\right),
$$


given any $y \geq x$, is nonincreasing in $x$. For any $x_{1} \leq x_{2} \leq \underline{x}$, it then follows that

$$
\max _{y \geq x_{2}} \bar{\Omega}\left(x_{2}, y\right)=\bar{\Omega}\left(x_{2}, y^{\star}\left(x_{2}\right)\right) \leq \bar{\Omega}\left(x_{1}, y^{\star}\left(x_{2}\right)\right) \leq \max _{y \geq x_{1}} \bar{\Omega}\left(x_{1}, y\right)
$$

here any $y^{\star}\left(x_{2}\right) \in \arg \max _{y \geq x_{2}} \bar{\Omega}\left(x_{2}, y\right)$ satisfies $y^{\star}\left(x_{2}\right) \geq x_{1}$. This implies that the objective value of the inner maximization problem $\max _{y \geq x} \bar{\Omega}(x, y)$ is nonincreasing on $x \leq \underline{x}$. Hence we have

$$
\min _{x \leq \underline{x}} \max \left\{\max _{y \geq x} \bar{\Omega}(x, y), \max _{y \leq x} \underline{\Omega}(x, y)\right\}=\min _{x \leq \underline{x}} \max _{y \geq x} \bar{\Omega}(x, y)=\max _{y \geq \underline{x}} \bar{\Omega}(\underline{x}, y),
$$

which certifies that $\underline{x}$ is the optimal decision in the region $x \leq \underline{x}$.

Step 2. Let $J=\max _{i \in \mathcal{N}}\left\{\bar{v}_{i} / \underline{u}_{i}\right\}$ and $K=M(p-c) /(L c)$ with $L=\sum_{i \in \mathcal{N}} \underline{u}_{i}$ and $M=\sum_{i \in[N] \backslash \mathcal{N}} \bar{v}_{i}$. We will show that

$$
\max \{J, K\} \in \underset{x \geq \max \{J, K\}}{\arg \min } \max \left\{\max _{y \geq x} \bar{\Omega}(x, y), \max _{y \leq x} \Omega(x, y)\right\}
$$

We first compute $\max _{y \leq x} \underline{\Omega}(x, y)$. Reading from Table 1 , for any fixed $x \geq \max \{J, K\}$ we have

$$
\begin{aligned}
\Omega(x, y) & =\frac{1}{N} \sum_{i \in \mathcal{N}: y>\underline{v}_{i} / \bar{u}_{i}}\left(-c \bar{u}_{i} y+c \bar{u}_{i} x\right)+\frac{1}{N} \sum_{i \in \mathcal{N}: y \leq \underline{v}_{i} / \bar{u}_{i}}\left((p-c) \bar{u}_{i} y+c \bar{u}_{i} x-p v\right) \\
& +\frac{1}{N} \sum_{i \in[N] \backslash \mathcal{N}: y>\underline{v}_{i} / \bar{u}_{i}}\left(-c \bar{u}_{i} y+c \bar{u}_{i} x\right)+\frac{1}{N} \sum_{i \in[N] \backslash \mathcal{N}: x \geq \underline{v}_{i} / \bar{u}_{i} \geq y}\left((p-c) \bar{u}_{i} y+c \bar{u}_{i} x-p v\right)^{+},
\end{aligned}
$$

which, given any $y \leq x$, is nondecreasing in $x$. For any $x_{2} \geq x_{1} \geq \max \{J, K\}$, it then follows that

$$
\max _{y \leq x_{1}} \Omega\left(x_{1}, y\right)=\underline{\Omega}\left(x_{1}, y^{\star}\left(x_{1}\right)\right) \leq \underline{\Omega}\left(x_{2}, y^{\star}\left(x_{1}\right)\right) \leq \max _{y \leq x_{2}} \Omega\left(x_{2}, y\right) ;
$$

here any $y^{\star}\left(x_{1}\right) \in \arg \max _{y \leq x_{1}} \Omega\left(x_{1}, y\right)$ satisfies $y^{\star}\left(x_{1}\right) \geq x_{2}$. This implies that the objective value of the maximization problem $\max _{y \leq x} \Omega(x, y)$ is nondecreasing on $x \geq \max \{J, K\}$. We then compute $\max _{y \geq x} \Omega(x, y)$. By Table 1 , for any fixed $x \geq J$ we have a function of $y$ on the domain $y \geq x$ :

$$
\begin{aligned}
\bar{\Omega}(x, y) & =\frac{1}{N} \sum_{i \in \mathcal{N}}\left(-c \underline{u}_{i} y+c \underline{u}_{i} x\right)+\frac{1}{N} \sum_{i \in[N] \backslash \mathcal{N}}\left(-(p-c) \frac{x \bar{v}_{i}}{y}+(p-c) \bar{v}_{i}\right) \\
& =\frac{1}{N}\left(L c x+M(p-c)-L c y-M(p-c) \frac{x}{y}\right) .
\end{aligned}
$$

Suppose that $K \geq J$. If $J \leq x \leq K$ then

$$
\max _{y \geq x} \bar{\Omega}(x, y)=\frac{1}{N}(L c x+M(p-c)-2 \sqrt{L M(p-c) c x}),
$$

where the maximum, achieved at $y=\sqrt{K x}$, is nonincreasing on $J \leq x \leq K$; whereas if $x \geq K=$ $\max \{J, K\}$ then the maximum, achieved at $y=x$, is always zero and thus is nondecreasing on $x \geq K$. Therefore, the worst-case regret

$$
\max \left\{\max _{y \geq x} \bar{\Omega}(x, y), \max _{y \leq x} \Omega(x, y)\right\}
$$


is nondecreasing on $x \geq K$, establishing that $x=K$ is optimal on the semi-interval $x \geq K$.

Suppose that $K \leq J$. The equality $\max _{y \geq x} \bar{\Omega}(x, y)=0$ holds for any $y \geq x \geq J \geq K$, with the maximum achieved at $y=x$. This implies that

$$
\min _{x \geq J} \max \left\{\max _{y \geq x} \bar{\Omega}(x, y), \max _{y \leq x} \Omega(x, y)\right\}=\min _{x \geq J} \max \left\{0, \max _{y \leq x} \Omega(x, y)\right\}=\min _{x \geq J} \max _{y \leq x} \Omega(x, y) .
$$

It is then not difficult to see $x=J$ is optimal on the semi-interval $x \geq J$ since $J=\max \{J, K\}$ and $\max _{y \leq x} \Omega(x, y)$ is nondecreasing on $x \geq J$.

Finally, we conclude that the optimal decision attaining the smallest worst-case regret on the semi-interval $x \geq \max \{J, K\}$ is always $\max \{J, K\}$.

Step 3. The preceding analysis transforms the minimax regret newsvendor model (5) into

$$
\min _{\underline{x} \leq x \leq \max \{J, K\}} \max \left\{\max _{y \geq x} \bar{\Omega}(x, y), \max _{y \leq x} \Omega(x, y)\right\} .
$$

According to Table 1 , if $y \leq \underline{x}$ then $\max _{u \in \mathcal{U}_{i}} \Lambda\left(x, y, u, \underline{v}_{i}\right)$ is always nondecreasing in $y$ for every $i \in[N]$. It follows that $\max _{y \leq \underline{x}} \underline{\Omega}(x, y)=\underline{\Omega}(x, \underline{x})$, which implies

$$
\max _{y \leq x} \underline{\Omega}(x, y) \leq \max _{\underline{x} \leq y \leq x} \underline{\Omega}(x, y) .
$$

It remains to show that, for any $\underline{x} \leq x \leq \max \{J, K\}$, the optimal solution to $\max _{y \geq x} \bar{\Omega}(x, y) \operatorname{must}$ satisfy $y \leq \max \{J, K\}$. Indeed, for any $\underline{x} \leq x \leq \max \{J, K\}$ and $y \geq \max \{J, K\}$ we have

$$
\bar{\Omega}(x, y)=\frac{1}{N}\left(C-L c y-M(p-c) \frac{x}{y}\right)
$$

where $C$ is a constant independent of $y$. Combining this observation and the solution structure of the optimization problem $\max _{y \geq x}\{-z y-x / y\}$ with nonnegative $x$ and $z$, we can tell that $\max _{y \geq \max \{J, K\}} \bar{\Omega}(x, y)=\bar{\Omega}(x, \max \{J, K\})$. Therefore,

$$
\max _{y \geq x} \bar{\Omega}(x, y)=\max _{x \leq y \leq \max \{J, K\}} \bar{\Omega}(x, y) .
$$

Finally, we complete the proof by setting $\bar{x}=\max \{J, K\}$.

Proof of Proposition 1. We proceed in three steps. We first rewrite the two components of the objective function of model (6), then we compare their values on the known boundary of the optimal order quantity, and finally, we explore the fact that their values must coincide at optimality.

Step 1. For any $i \in[N]$ and any fixed $x$ and $u$, Lemma 1 implies that $\Lambda\left(x, y, u, \bar{v}_{i}\right) \geq \Lambda\left(x, y, u, \underline{v}_{i}\right)$ if $y \geq x$; whereas $\Lambda\left(x, y, u, \bar{v}_{i}\right) \leq \Lambda\left(x, y, u, \underline{v}_{i}\right)$ if $y \leq x$. Then for any fixed $x \in[\underline{x}, \bar{x}]$ it follows that

$$
\max \left\{\sum_{i \in[N]} \max _{u \in \mathcal{U}_{i}} \Lambda\left(x, y, u, \bar{v}_{i}\right), \sum_{i \in[N]} \max _{u \in \mathcal{U}_{i}} \Lambda\left(x, y, u, \underline{v}_{i}\right)\right\}= \begin{cases}\sum_{i \in[N]} \max _{u \in \mathcal{U}_{i}} \Lambda\left(x, y, u, \bar{v}_{i}\right) & \forall x \leq y \leq \bar{x}, \\ \sum_{i \in[N]} \max _{u \in \mathcal{U}_{i}} \Lambda\left(x, y, u, \underline{v}_{i}\right) & \forall \underline{x} \leq y \leq x,\end{cases}
$$


implying that for any fixed $x \in[\underline{x}, \bar{x}]$ we have

$$
\begin{aligned}
& \max _{x \leq y \leq \bar{x}} \sum_{i \in[N]} \max _{u \in \mathcal{U}_{i}} \Lambda\left(x, y, u, \bar{v}_{i}\right) \geq \max _{x \leq y \leq \bar{x}} \sum_{i \in[N]} \max _{u \in \mathcal{U}_{i}} \Lambda\left(x, y, u, \underline{v}_{i}\right) ; \\
& \max _{\underline{x} \leq y \leq x} \sum_{i \in[N]} \max _{u \in \mathcal{U}_{i}} \Lambda\left(x, y, u, \underline{v}_{i}\right) \geq \max _{\underline{x} \leq y \leq x} \sum_{i \in[N]} \max _{u \in \mathcal{U}_{i}} \Lambda\left(x, y, u, \bar{v}_{i}\right) .
\end{aligned}
$$

With these inequalities, the objective function of model (6), multiplied by $N$, can be rewritten as

$$
\begin{aligned}
& \max \left\{\max _{x \leq y \leq \bar{x}} \sum_{i \in[N]} \max _{u \in \mathcal{U}_{i}} \Lambda\left(x, y, u, \bar{v}_{i}\right), \max _{\underline{x} \leq y \leq x} \sum_{i \in[N]} \max _{u \in \mathcal{U}_{i}} \Lambda\left(x, y, u, \underline{v}_{i}\right)\right\} \\
& =\max \left\{\begin{array}{l}
\max _{x \leq y \leq \bar{x}} \sum_{i \in[N]} \max _{u \in \mathcal{U}_{i}} \Lambda\left(x, y, u, \bar{v}_{i}\right), \max _{x \leq y \leq \bar{x}} \sum_{i \in[N]} \max _{u \in \mathcal{U}_{i}} \Lambda\left(x, y, u, \underline{v}_{i}\right), \\
\max _{\underline{x} \leq y \leq x} \sum_{i \in[N]} \max _{u \in \mathcal{U}_{i}} \Lambda\left(x, y, u, \bar{v}_{i}\right), \max _{\underline{x} \leq y \leq x} \sum_{i \in[N]} \max _{u \in \mathcal{U}_{i}} \Lambda\left(x, y, u, \underline{v}_{i}\right)
\end{array}\right\} \\
& =\max \left\{\max _{\underline{x} \leq y \leq \bar{x}} \sum_{i \in[N]} \max _{u \in \mathcal{U}_{i}} \Lambda\left(x, y, u, \bar{v}_{i}\right), \max _{\underline{x} \leq y \leq \bar{x}} \sum_{i \in[N]} \max _{u \in \mathcal{U}_{i}} \Lambda\left(x, y, u, \underline{v}_{i}\right)\right\},
\end{aligned}
$$

where in the last equality, the two components

$$
\max _{\underline{x} \leq y \leq \bar{x}} \sum_{i \in[N]} \max _{u \in \mathcal{U}_{i}} \Lambda\left(x, y, u, \bar{v}_{i}\right) \quad \text { and } \max _{\underline{x} \leq y \leq \bar{x}} \sum_{i \in[N]} \max _{u \in \mathcal{U}_{i}} \Lambda\left(x, y, u, \underline{v}_{i}\right)
$$

are each a convex function of $x$ because the function $\Lambda(x, y, u, v)$ is convex in $x$ for any fixed values of $y, u$, and $v$. The preceding argument implies that problem (6) is equivalent to

$$
\min _{\underline{x} \leq x \leq \bar{x}} \max \left\{\max _{\underline{x} \leq y \leq \bar{x}} \frac{1}{N} \sum_{i \in[N]} \max _{u \in \mathcal{U}_{i}} \Lambda\left(x, y, u, \bar{v}_{i}\right), \max _{\underline{x} \leq y \leq \bar{x}} \frac{1}{N} \sum_{i \in[N]} \max _{u \in \mathcal{U}_{i}} \Lambda\left(x, y, u, \underline{v}_{i}\right)\right\} .
$$

Step 2. Since $\Lambda(x, y, u, v)$ is continuous and $\left\{\mathcal{U}_{i}\right\}_{i \in[N]}$ and $[\underline{x}, \bar{x}]$ are compact, by Berge's maximum theorem (Ausubel and Deneckere 1993), the two functions in (20) are continuous. Besides, we have

$$
\lim _{x \rightarrow \underline{x}} \max _{\underline{x} \leq y \leq x} \sum_{i \in[N]} \max _{u \in \mathcal{U}_{i}} \Lambda\left(x, y, u, \underline{v}_{i}\right)=0 \quad \text { and } \quad \lim _{x \rightarrow \underline{x}} \max _{\underline{x} \leq y \leq x} \sum_{i \in[N]} \max _{u \in \mathcal{U}_{i}} \Lambda\left(x, y, u, \bar{v}_{i}\right)=0 .
$$

From inequalities (19) we obtain

$$
\begin{aligned}
& \lim _{x \rightarrow \underline{x}} \max _{\underline{x} \leq y \leq \bar{x}} \sum_{i \in[N]} \max _{u \in \mathcal{U}_{i}} \Lambda\left(x, y, u, \underline{v}_{i}\right) \\
& =\max \left\{\lim _{x \rightarrow \underline{x}} \max _{x \leq y \leq \bar{x}} \sum_{i \in[N]} \max _{u \in \mathcal{U}_{i}} \Lambda\left(x, y, u, \underline{v}_{i}\right), \lim _{x \rightarrow \underline{x}} \max _{\underline{x} \leq y \leq x} \sum_{i \in[N]} \max _{u \in \mathcal{U}_{i}} \Lambda\left(x, y, u, \underline{v}_{i}\right)\right\} \\
& <\max \left\{\lim _{x \rightarrow \underline{x}} \max _{x \leq y \leq \bar{x}} \sum_{i \in[N]} \max _{u \in \mathcal{U}_{i}} \Lambda\left(x, y, u, \bar{v}_{i}\right), 0\right\} \\
& =\max \left\{\lim _{x \rightarrow \underline{x}} \max _{x \leq y \leq \bar{x}} \sum_{i \in[N]} \max _{u \in \mathcal{U}_{i}} \Lambda\left(x, y, u, \bar{v}_{i}\right), \lim _{x \rightarrow \underline{x}} \max _{\underline{x} \leq y \leq x} \sum_{i \in[N]} \max _{u \in \mathcal{U}_{i}} \Lambda\left(x, y, u, \bar{v}_{i}\right)\right\} \\
& =\lim _{x \rightarrow \underline{x}} \max _{\underline{x} \leq y \leq \bar{x}} \sum_{i \in[N]} \max _{u \in \mathcal{U}_{i}} \Lambda\left(x, y, u, \bar{v}_{i}\right)
\end{aligned}
$$


where the second equality follows from the continuity of piecewise maximum of two continuous functions, the strict inequality is due to inequalities (19), identities (22), and $\theta>0$ so that $\bar{u}_{i}>\underline{u}_{i}$ and $\bar{x}>\underline{x}$, and the third equality is because of identities (22). By similar arguments, we have

$$
\lim _{x \rightarrow \bar{x}} \max _{\underline{x} \leq y \leq \bar{x}} \sum_{i \in[N]} \max _{u \in \mathcal{U}_{i}} \Lambda\left(x, y, u, \underline{v}_{i}\right)>\lim _{x \rightarrow \bar{x}} \max _{\underline{x} \leq y \leq \bar{x}} \sum_{i \in[N]} \max _{u \in \mathcal{U}_{i}} \Lambda\left(x, y, u, \bar{v}_{i}\right)
$$

Step 3. With the convexity and continuity of the two functions in (20) and the facts in Step 2, we conclude that the optimal minimax regret order quantity $x_{\mathrm{R}}^{\star}$ of problem (21) must satisfy

$$
\max _{\underline{x} \leq y \leq \bar{x}} \sum_{i \in[N]} \max _{u \in \mathcal{U}_{i}} \Lambda\left(x_{\mathrm{R}}^{\star}, y, u, \bar{v}_{i}\right)=\max _{\underline{x} \leq y \leq \bar{x}} \sum_{i \in[N]} \max _{u \in \mathcal{U}_{i}} \Lambda\left(x_{\mathrm{R}}^{\star}, y, u, \underline{v}_{i}\right) .
$$

In other words, the following identity holds:

$$
\begin{aligned}
& \max \{\underbrace{\max _{\underline{x} \leq y \leq x_{\mathrm{R}}^{\star}} \sum_{i \in[N]} \max _{u \in \mathcal{U}_{i}} \Lambda\left(x_{\mathrm{R}}^{\star}, y, u, \underline{v}_{i}\right)}_{\triangleq \mathrm{A}}, \underbrace{\max _{x_{\mathrm{R}}^{\star} \leq y \leq \bar{x}} \sum_{i \in[N]} \max _{u \in \mathcal{U}_{i}} \Lambda\left(x_{\mathrm{R}}^{\star}, y, u, \underline{v}_{i}\right)}_{\triangleq \mathrm{B}}\} \\
& =\max \{\underbrace{\max _{\underline{x} \leq y \leq x_{\mathrm{R}}^{\star}} \sum_{i \in[N]} \max _{u \in \mathcal{U}_{i}} \Lambda\left(x_{\mathrm{R}}^{\star}, y, u, \bar{v}_{i}\right)}_{\triangleq \mathrm{C}} \underbrace{\max _{x_{\mathrm{R}}^{\star} \leq y \leq \bar{x}} \sum_{i \in[N]} \max _{u \in \mathcal{U}_{i}} \Lambda\left(x_{\mathrm{R}}^{\star}, y, u, \bar{v}_{i}\right)}_{\triangleq_{\mathrm{D}}}\} .
\end{aligned}
$$

Together with inequalities (19) that states $\mathrm{A} \geq \mathrm{C}$ and $\mathrm{B} \leq \mathrm{D}$, the identity (23) implies: (i) $\mathrm{C} \leq \mathrm{A} \leq$ $\mathrm{B}=\mathrm{D}$; (ii) $\mathrm{B} \leq \mathrm{D} \leq \mathrm{A}=\mathrm{C}$; or (iii) $\mathrm{B}, \mathrm{C} \leq \mathrm{A}=\mathrm{D}$. It remains to show that Case (i) or (ii) will lead to $\mathrm{A}=\mathrm{D}$. For ease of notation, we let $y_{\mathrm{A}}^{\star}, y_{\mathrm{B}}^{\star}, y_{\mathrm{C}}^{\star}, y_{\mathrm{D}}^{\star}$ be the optimal solutions achieving A, B, C, D.

Case (i). Suppose $\mathrm{C} \leq \mathrm{A} \leq \mathrm{B}=\mathrm{D}$. Without loss of generality, we assume $\mathrm{B}=\mathrm{D}>0$ because otherwise $\mathrm{C}=\mathrm{A}=\mathrm{B}=\mathrm{D}=0$ and we are done. For any $i \in[N]$ and any fixed $u$, Lemma 1 implies that $\Lambda\left(x_{\mathrm{R}}^{\star}, y, u, \bar{v}_{i}\right) \geq \Lambda\left(x_{\mathrm{R}}^{\star}, y, u, \underline{v}_{i}\right)$ when $y \geq x_{\mathrm{R}}^{\star}$ and equality holds either $y=x_{\mathrm{R}}^{\star}$, or $u y \leq \underline{v}_{i}$, or $u x_{\mathrm{R}}^{\star} \geq \bar{v}_{i}$. The relation $\mathrm{B}=\mathrm{D}$ implies that for each $i \in[N]$, either $y_{\mathrm{B}}^{\star}, y_{\mathrm{D}}^{\star} \in\left[x_{\mathrm{R}}^{\star}, \bar{x}\right]$ satisfying $\bar{u}_{i} y_{\mathrm{B}}^{\star} \leq \underline{v}_{i}$ and $\bar{u}_{i} y_{\mathrm{D}}^{\star} \leq \underline{v}_{i}$ or $\underline{u}_{i} x_{\mathrm{R}}^{\star} \geq \bar{v}_{i}$. This gives a (decision-dependent) partition of $[N]: \mathcal{N} \triangleq\left\{i \in[N]: \underline{v}_{i} \geq\right.$ $\left.\bar{u}_{i} y_{\mathrm{B}}^{\star} \geq \bar{u}_{i} x_{\mathrm{R}}^{\star}, \underline{v}_{i} \geq \bar{u}_{i} y_{\mathrm{D}}^{\star} \geq \bar{u}_{i} x_{\mathrm{R}}^{\star}\right\}$ and $\overline{\mathcal{N}} \triangleq\left\{i \in[N]: \bar{v}_{i} \leq \underline{u}_{i} x_{\mathrm{R}}^{\star} \leq \underline{u}_{i} y_{\mathrm{B}}^{\star}, \bar{v}_{i} \leq \underline{u}_{i} x_{\mathrm{R}}^{\star} \leq \underline{u}_{i} y_{\mathrm{D}}^{\star}\right\}$. According to Table 1 , the relation $\mathrm{B}=\mathrm{D}$ can be simplified as

$$
\mathrm{B}=\mathrm{D}=\Psi_{\mathrm{D}}\left(x_{\mathrm{R}}^{\star}\right) \triangleq\left\{\begin{array}{l}
\max _{y_{\mathrm{D}}}\left((p-c) \sum_{i \in \mathcal{N}} \bar{u}_{i}-c \sum_{i \in \mathcal{N}} \underline{u}_{i}\right)\left(y_{\mathrm{D}}-x_{\mathrm{R}}^{\star}\right) \\
\text { s.t. } \bar{u}_{i} y_{\mathrm{D}} \leq \underline{v}_{i} \quad \forall i \in \mathcal{N} \\
\underline{u}_{i} x_{\mathrm{R}}^{\star} \geq \bar{v}_{i} \quad \forall i \in \overline{\mathcal{N}} \\
y_{\mathrm{D}} \geq x_{\mathrm{R}}^{\star} .
\end{array}\right.
$$


Similarly, by definitions of sets $\mathcal{N}$ and $\overline{\mathcal{N}}$ and another set $\mathcal{N}^{\dagger}=\left\{i \in \overline{\mathcal{N}}: y_{\mathrm{A}} \leq \underline{v}_{i} / \bar{u}_{i}\right\}$, we have

$$
\mathrm{C} \leq \mathrm{A}=\Psi_{\mathrm{A}}\left(x _ { \mathrm { R } } ^ { \star } \triangleq \left\{\begin{array}{ll}
\max _{y_{\mathrm{A}}}\left\{\left((p-c) \sum_{i \in \mathcal{N}} \underline{u}_{i}-c \sum_{i \in \overline{\mathcal{N}}} \bar{u}_{i}\right)\left(y_{\mathrm{A}}-x_{\mathrm{R}}^{\star}\right)+p \sum_{i \in \mathcal{N}^{\dagger}}\left(\bar{u}_{i} y_{\mathrm{A}}-\underline{v}_{i}\right)\right\} \\
\text { s.t. } \bar{u}_{i} x_{\mathrm{R}}^{\star} \leq \underline{v}_{i} \quad \forall i \in \mathcal{N} \\
\underline{u}_{i} x_{\mathrm{R}}^{\star} \geq \bar{v}_{i} \quad \forall i \in \overline{\mathcal{N}} \\
\bar{u}_{i} y_{\mathrm{A}} \leq \underline{v}_{i} & \forall i \in \mathcal{N}^{\dagger} \\
\bar{u}_{i} y_{\mathrm{A}} \geq \underline{v}_{i} & \forall i \in \overline{\mathcal{N}} \backslash \mathcal{N}^{\dagger} \\
y_{\mathrm{A}} \leq x_{\mathrm{R}}^{\star} . &
\end{array}\right.\right.
$$

Since each of $\Psi_{\mathrm{A}}\left(x_{\mathrm{R}}^{\star}\right)$ and $\Psi_{\mathrm{D}}\left(x_{\mathrm{R}}^{\star}\right)$ is the maximum of a finite number of one-dimensional linear programs, they are convex and continuous in $x_{\mathrm{R}}^{\star}$. On the other hand, problem (21) is equivalent to

$$
\begin{aligned}
\min _{x_{\mathrm{R}}^{\star}} \max & \left\{\frac{1}{N} \Psi_{\mathrm{A}}\left(x_{\mathrm{R}}^{\star}\right), \frac{1}{N} \Psi_{\mathrm{D}}\left(x_{\mathrm{R}}^{\star}\right)\right\} \\
\text { s.t. } & \bar{u}_{i} x_{\mathrm{R}}^{\star} \leq \underline{v}_{i} \quad \forall i \in \mathcal{N} \\
\underline{u}_{i} x_{\mathrm{R}}^{\star} & \geq \bar{v}_{i} \quad \forall i \in \overline{\mathcal{N}},
\end{aligned}
$$

where the feasible region is an interval from $\max _{i \in \mathcal{N}}\left\{\bar{v}_{i} / \underline{u}_{i}\right\}$ to $\min _{i \in \mathcal{N}}\left\{\underline{v}_{i} / \bar{u}_{i}\right\}$. On the right end, objective values of the two convex and continuous functions $\Psi_{\mathrm{A}}\left(x_{\mathrm{R}}^{\star}\right)$ and $\Psi_{\mathrm{D}}\left(x_{\mathrm{R}}^{\star}\right)$ satisfy:

$$
\lim _{x_{\mathrm{R}}^{\star} \rightarrow \min _{i \in \mathcal{N}}\left\{\underline{v}_{i} / \bar{u}_{i}\right\}} \Psi_{\mathrm{D}}\left(x_{\mathrm{R}}^{\star}\right)=0 \leq \lim _{x_{\mathrm{R}}^{\star} \rightarrow \min _{i \in \mathcal{N}}\left\{\underline{v}_{i} / \bar{u}_{i}\right\}} \Psi_{\mathrm{A}}\left(x_{\mathrm{R}}^{\star}\right) .
$$

Thus, at optimality, we must have $\mathrm{A} \geq \mathrm{D}$, implying $\mathrm{A}=\mathrm{B}=\mathrm{D}$ with our presumed $\mathrm{C} \leq \mathrm{A} \leq \mathrm{B}=\mathrm{D}$.

Case (ii). Suppose $\mathrm{B} \leq \mathrm{D} \leq \mathrm{A}=\mathrm{C}$. Similarly, we assume $\mathrm{A}=\mathrm{C}>0$ without loss of generality. For any $i \in[N]$ and any fixed $u$, Lemma 1 implies that $\Lambda\left(x_{\mathrm{R}}^{\star}, y, u, \bar{v}_{i}\right) \leq \Lambda\left(x_{\mathrm{R}}^{\star}, y, u, \underline{v}_{i}\right)$ when $y \leq x_{\mathrm{R}}^{\star}$ and equality holds either $y=x_{\mathrm{R}}^{\star}$, or $u y \geq \bar{v}_{i}$, or $u x_{\mathrm{R}}^{\star} \leq \underline{v}_{i}$. The relation $\mathrm{A}=\mathrm{C}$ implies that for each $i \in[N]$, either $y_{\mathrm{A}}^{\star}, y_{\mathrm{C}}^{\star} \in\left[\underline{x}, x_{\mathrm{R}}^{\star}\right]$ satisfying $\underline{u}_{i} y_{\mathrm{A}}^{\star} \geq \bar{v}_{i}$ and $\underline{u}_{i} y_{\mathrm{C}}^{\star} \geq \bar{v}_{i}$ or $\bar{u}_{i} x_{\mathrm{R}}^{\star} \leq \underline{v}_{i}$. This gives the (decision-dependent) partition of $[N]: \underline{\mathcal{N}} \triangleq\left\{i \in[N]: \underline{v}_{i} \geq \bar{u}_{i} x_{\mathrm{R}}^{\star} \geq \bar{u}_{i} y_{\mathrm{A}}^{\star}, \underline{v}_{i} \geq \bar{u}_{i} x_{\mathrm{R}}^{\star} \geq \bar{u}_{i} y_{\mathrm{C}}^{\star}\right\}$ and $\overline{\mathcal{N}} \triangleq\left\{i \in[N]: \bar{v}_{i} \leq \underline{u}_{i} y_{\mathrm{A}}^{\star} \leq \underline{u}_{i} x_{\mathrm{R}}^{\star}, \bar{v}_{i} \leq \underline{u}_{i} y_{\mathrm{C}}^{\star} \leq \underline{u}_{i} x_{\mathrm{R}}^{\star}\right\}$. With Table 1 , we simplify the relation $\mathrm{A}=\mathrm{C}$ as

$$
\mathrm{A}=\mathrm{C}=\Psi_{\mathrm{A}}\left(x_{\mathrm{R}}^{\star}\right) \triangleq\left\{\begin{array}{l}
\max _{y_{\mathrm{A}}}\left((p-c) \sum_{i \in \mathcal{N}} \underline{u}_{i}-c \sum_{i \in \overline{\mathcal{N}}} \bar{u}_{i}\right)\left(y_{\mathrm{A}}-x_{\mathrm{R}}^{\star}\right) \\
\text { s.t. } \bar{u}_{i} x_{\mathrm{R}}^{\star} \leq \underline{v}_{i} \quad \forall i \in \mathcal{\mathcal { N }} \\
\underline{u}_{i} y_{\mathrm{A}} \geq \bar{v}_{i} \quad \forall i \in \overline{\mathcal{N}} \\
y_{\mathrm{A}} \leq x_{\mathrm{R}}^{\star} .
\end{array}\right.
$$

By realizing that the optimal decision takes $y_{\mathrm{A}}^{\star}=x_{\mathrm{R}}^{\star}$ if $(p-c) \sum_{i \in \mathcal{N}} \underline{u}_{i}-c \sum_{i \in \overline{\mathcal{N}}} \bar{u}_{i} \geq 0$ and takes $y_{\mathrm{A}}^{\star}=\max _{i \in \overline{\mathcal{N}}}\left\{\bar{v}_{i} / \underline{u}_{i}\right\}$ otherwise, we have

$$
\Psi_{\mathrm{A}}\left(x_{\mathrm{R}}^{\star}\right)=\left(-(p-c) \sum_{i \in \mathcal{N}} \underline{u}_{i}+c \sum_{i \in \overline{\mathcal{N}}} \bar{u}_{i}\right)^{+}\left(x_{\mathrm{R}}^{\star}-\max _{i \in \mathcal{N}}\left\{\bar{v}_{i} / \underline{u}_{i}\right\}\right),
$$


which is a nondecreasing affine function in the interval from $\max _{i \in \overline{\mathcal{N}}}\left\{\bar{v}_{i} / \underline{u}_{i}\right\}$ to $\min _{i \in \mathcal{N}}\left\{\underline{v}_{i} / \bar{u}_{i}\right\}$. Similarly, by definitions of sets $\mathcal{N}$ and $\overline{\mathcal{N}}$ and another three sets $\mathcal{N}_{1}^{\dagger}=\left\{i \in \mathcal{N}: \bar{u}_{i} y_{\mathrm{D}} \leq \bar{v}_{i}\right\}, \mathcal{N}_{2}^{\dagger}=$ $\left\{i \in \mathcal{N}: \underline{u}_{i} y_{\mathrm{D}} \leq \bar{v}_{i}, \bar{u}_{i} y_{\mathrm{D}} \geq \bar{v}_{i}\right\}$, and $\mathcal{N}_{3}^{\dagger}=\left\{i \in \underline{\mathcal{N}}: \underline{u}_{i} y_{\mathrm{D}} \geq \bar{v}_{i}\right\}$ that we break ties arbitrarily, we have

$$
\mathrm{B} \leq \mathrm{D}=\Psi_{\mathrm{D}}\left(x_{\mathrm{R}}^{\star}\right) \triangleq\left\{\begin{aligned}
& \max _{y_{\mathrm{D}}}\left\{(p-c) \sum_{i \in \mathcal{N}_{1}^{\dagger}} \bar{u}_{i}\left(y_{\mathrm{D}}-x_{\mathrm{R}}^{\star}\right)-c \sum_{i \in \overline{\mathcal{N}}} \underline{u}_{i}\left(y_{\mathrm{D}}-x_{\mathrm{R}}^{\star}\right)\right. \\
&\left.+(p-c) \sum_{i \in \mathcal{N}_{2}^{\dagger}} \bar{v}_{i}\left(1-x_{\mathrm{R}}^{\star} / y_{\mathrm{D}}\right)+\sum_{i \in \mathcal{N}_{3}^{\dagger}}\left(p \bar{v}_{i}-c \underline{u}_{i} y_{\mathrm{D}}-(p-c) \underline{u}_{i} x_{\mathrm{R}}^{\star}\right)\right\} \\
& \text { s.t. } \bar{u}_{i} x_{\mathrm{R}}^{\star} \leq \underline{v}_{i} \quad \forall i \in \mathcal{N} \\
& \underline{u}_{i} x_{\mathrm{R}}^{\star} \geq \bar{v}_{i} \quad \forall i \in \overline{\mathcal{N}} \\
& \bar{u}_{i} y_{\mathrm{D}} \leq \bar{v}_{i} \quad \forall i \in \mathcal{N}_{1}^{\dagger} \\
& \underline{u}_{i} y_{\mathrm{D}} \leq \bar{v}_{i} \quad \forall i \in \mathcal{N}_{2}^{\dagger} \\
& \bar{u}_{i} y_{\mathrm{D}} \geq \bar{v}_{i} \quad \forall i \in \mathcal{N}_{2}^{\dagger} \\
& \underline{u}_{i} y_{\mathrm{D}} \geq \bar{v}_{i} \quad \forall i \in \mathcal{N}_{3}^{\dagger} \\
& y_{\mathrm{D}} \geq x_{\mathrm{R}}^{\star} .
\end{aligned}\right.
$$

In view of the above reformulation, with Berge's maximum theorem (Ausubel and Deneckere 1993) we see that $\Psi_{\mathrm{D}}\left(x_{\mathrm{R}}^{\star}\right)$ is continuous in the compact interval from $\max _{i \in \mathcal{N}}\left\{\bar{v}_{i} / \underline{u}_{i}\right\}$ to $\min _{i \in \mathcal{N}}\left\{\underline{v}_{i} / \bar{u}_{i}\right\}$. On the left end, objective values of the two continuous functions $\Psi_{\mathrm{A}}\left(x_{\mathrm{R}}^{\star}\right)$ and $\Psi_{\mathrm{D}}\left(x_{\mathrm{R}}^{\star}\right)$ satisfy:

$$
\lim _{x_{\mathrm{R}}^{\star} \rightarrow \max _{i \in \overline{\mathcal{N}}^{\prime}}\left\{\bar{v}_{i} / \underline{u}_{i}\right\}} \Psi_{\mathrm{A}}\left(x_{\mathrm{R}}^{\star}\right)=0 \leq \lim _{x_{\mathrm{R}}^{\star} \rightarrow \max _{i \in \mathcal{N}}\left\{\bar{v}_{i} / \underline{u}_{i}\right\}} \Psi_{\mathrm{D}}\left(x_{\mathrm{R}}^{\star}\right) .
$$

Since $\Psi_{\mathrm{A}}\left(x_{\mathrm{R}}^{\star}\right)$ is a nondecreasing affine function and $\Psi_{\mathrm{D}}\left(x_{\mathrm{R}}^{\star}\right)$ is continuous at their common domain, at optimality, we must have $\mathrm{D} \geq \mathrm{A}$, implying $\mathrm{A}=\mathrm{C}=\mathrm{D}$ with our presumed $\mathrm{B} \leq \mathrm{D} \leq \mathrm{A}=\mathrm{C}$.

The proof now concludes since we have $\mathrm{A}=\mathrm{D}$ in all three cases.

Proof of Proposition 2. From reformulation (6) we know that the optimal worst-case regret is neither larger than the regret of decision $\underline{x}, \gamma(\underline{x})=\max _{\underline{x} \leq y \leq \bar{x}} \frac{1}{N} \sum_{i \in[N]} \max _{\underline{u}_{i} \leq u \leq \bar{u}_{i}} \Lambda\left(\underline{x}, y, u, \bar{v}_{i}\right)$, nor larger than the regret of decision $\bar{x}, \gamma(\bar{x})=\max _{\underline{x} \leq y \leq \bar{x}} \frac{1}{N} \sum_{i \in[N]} \max _{\underline{u}_{i} \leq u \leq \bar{u}_{i}} \Lambda\left(\bar{x}, y, u, \underline{v}_{i}\right)$.

We first bound $\gamma(\underline{x})$ from above. By the definition of $\underline{x}=\min _{i \in[N]}\left\{\underline{v}_{i} / \bar{u}_{i}\right\}$, for any $i \in[N]$ and any $\underline{u}_{i} \leq u \leq \bar{u}_{i}$ we have

$$
\begin{aligned}
\Lambda\left(\underline{x}, y, u, \bar{v}_{i}\right) & =(p-c) u y-p\left(u y-\bar{v}_{i}\right)^{+}-(p-c) u \underline{x} \\
& \leq(p-c) u y-p\left(u y-\bar{v}_{i}\right)^{+}-\left((p-c) \underline{u}_{i} \underline{x}-p\left(\underline{u}_{i} \underline{x}-\bar{v}_{i}\right)^{+}\right) .
\end{aligned}
$$


The Lipschitz constant of a piecewise affine function $(p-c) z-p z^{+}$is $\max \{p-c, c\}$, so

$$
\begin{aligned}
\gamma(\underline{x}) & =\max _{\underline{x} \leq y \leq \bar{x}} \frac{1}{N} \sum_{i \in[N]} \max _{\underline{u}_{i} \leq u \leq \bar{u}_{i}} \Lambda\left(\underline{x}, y, u, \bar{v}_{i}\right) \\
& \leq \frac{1}{N} \sum_{i \in[N]} \max _{\underline{x} \leq y \leq \bar{x}, \underline{u}_{i} \leq u \leq \bar{u}_{i}}\left\{(p-c) u y-p\left(u y-\bar{v}_{i}\right)^{+}-\left((p-c) \underline{u}_{i} \underline{x}-p\left(\underline{u}_{i} \underline{x}-\bar{v}_{i}\right)^{+}\right)\right\} \\
& \leq \frac{1}{N} \sum_{i \in[N]} \max \{p-c, c\} \cdot \max _{\underline{x} \leq y \leq \bar{x}, \underline{u}_{i} \leq u \leq \bar{u}_{i}}\left|y u-x \underline{u}_{i}\right| \\
& \leq \max \{p-c, c\} \cdot(\bar{x}-\underline{x}) \cdot(2 \theta) .
\end{aligned}
$$

We next bound $\gamma(\bar{x})$ from above. By the definition of $\bar{x}$, for any $i \in \mathcal{N}$ we have

$$
\begin{aligned}
\Lambda\left(\bar{x}, y, u, \underline{v}_{i}\right) & =(p-c) u y-p\left(u y-\bar{v}_{i}\right)^{+}+c u \bar{x}-p \bar{v}_{i} \\
& \leq(p-c) u y-p\left(u y-\bar{v}_{i}\right)^{+}-\left((p-c) \bar{u}_{i} \bar{x}-p\left(\bar{u}_{i} \bar{x}-\bar{v}_{i}\right)^{+}\right)
\end{aligned}
$$

whereas for any $i \in[N] \backslash \mathcal{N}$, we have

$$
\Lambda\left(\bar{x}, y, u, \underline{v}_{i}\right)= \begin{cases}(p-c) u y-(p-c) u \bar{x} \leq(p-c) u y-(p-c) \underline{u}_{i} \bar{x} & \text { if } \underline{v}_{i} \geq u \bar{x} \\ (p-c) u y-(p-c) u \bar{x}+p\left(u \bar{x}-\underline{v}_{i}\right) \leq-c \underline{u}_{i} y+c u \bar{x} & \text { if } u y \leq \underline{v}_{i} \leq u \bar{x} \\ -c u y+c u \bar{x} \leq-c \underline{u}_{i} y+c u \bar{x} & \text { if } \underline{v}_{i} \leq u y,\end{cases}
$$

which is not larger than $\max \left\{(p-c) u y-(p-c) \underline{u}_{i} \bar{x},-c \underline{u}_{i} y+c u \bar{x}\right\}$ for any $\underline{u}_{i} \leq u \leq \bar{u}_{i}$. Since the Lipschitz constant of an affine function $a z$ is $a$, by similar arguments we obtain $\gamma(\underline{x}) \leq 2(\bar{x}-$ $\underline{x}) \max \{p-c, c\} \theta$. The bounds on regrets, i.e., $\gamma(\underline{x})$ and $\gamma(\bar{x})$, coincide, concluding the proof.

Proof of Theorem 2. We proceed in three steps. First, we prove that

$$
\underline{x}^{\star} \in \underset{x \leq \underline{x}^{\star}}{\arg \min } \max \left\{\max _{y \geq x} \mathbb{E}_{\overline{\mathbb{P}}}[V(y, \tilde{v})-V(x, \tilde{v})], \max _{y \leq x} \mathbb{E}_{\mathbb{P}}[V(y, \tilde{v})-V(x, \tilde{v})]\right\} .
$$

Indeed, for any $x \leq \underline{x}^{\star}$ we observe that $\max _{y \geq x} \mathbb{E}_{\overline{\mathbb{P}}}[V(y, \tilde{v})-V(x, \tilde{v})]=\mathbb{E}_{\overline{\mathbb{P}}}\left[V\left(\bar{x}^{\star}, \tilde{v}\right)-V(x, \tilde{v})\right]$ and $\max _{y \leq x} \mathbb{E}_{\mathbb{P}}[V(y, \tilde{v})-V(x, \tilde{v})]=0$ by selecting $y=x$. Hence for any $x \leq \underline{x}^{\star}$ we have

$$
\max \left\{\max _{y \geq x} \mathbb{E}_{\overline{\mathbb{P}}}[V(y, \tilde{v})-V(x, \tilde{v})], \max _{y \leq x} \mathbb{E}_{\mathbb{P}}[V(y, \tilde{v})-V(x, \tilde{v})]\right\}=\mathbb{E}_{\overline{\mathbb{P}}}\left[V\left(\bar{x}^{\star}, \tilde{v}\right)-V(x, \tilde{v})\right],
$$

which is decreasing in $x \leq \underline{x}^{\star}$ owing to concavity of the univariate function $\mathbb{E}_{\overline{\mathbb{P}}}[V(x, \tilde{v})]$ and to $\bar{x}^{\star} \in$ $\arg \max _{x} \mathbb{E}_{\overline{\mathbb{P}}}[V(x, \tilde{v})]$ and $\underline{x}^{\star} \leq \bar{x}^{\star}$. So in the semi-interval $x \leq \underline{x}^{\star}$, the function $\mathbb{E}_{\overline{\mathbb{P}}}\left[V\left(\bar{x}^{\star}, \tilde{v}\right)-V(x, \tilde{v})\right]$ is nondecreasing, which means that optimal decision is $\underline{x}^{\star}$.

Second, similar arguments enable us to prove that

$$
\bar{x}^{\star} \in \underset{x \geq \bar{x}^{\star}}{\arg \min } \max \left\{\max _{y \geq x} \mathbb{E}_{\overline{\mathbb{P}}}[V(y, \tilde{v})-V(x, \tilde{v})], \max _{y \leq x} \mathbb{E}_{\mathbb{P}}[V(y, \tilde{v})-V(x, \tilde{v})]\right\} .
$$

Indeed, for any $x \geq \bar{x}^{\star}$, we observe that $\max _{y \geq x} \mathbb{E}_{\overline{\mathbb{P}}}[V(y, \tilde{v})-V(x, \tilde{v})]=0$ (by selecting $y=x$ ) and that $\max _{y \leq x} \mathbb{E}_{\mathbb{P}}[V(y, \tilde{v})-V(x, \tilde{v})]=\mathbb{E}_{\mathbb{P}}\left[V\left(\underline{x}^{\star}, \tilde{v}\right)-V(x, \tilde{v})\right]$. It follows that, for any $x \geq \bar{x}^{\star}$,

$$
\max \left\{\max _{y \geq x} \mathbb{E}_{\overline{\mathbb{P}}}[V(y, \tilde{v})-V(x, \tilde{v})], \max _{y \leq x} \mathbb{E}_{\mathbb{P}}[V(y, \tilde{v})-V(x, \tilde{v})]\right\}=\mathbb{E}_{\underline{\mathbb{P}}}\left[V\left(\underline{x}^{\star}, \tilde{v}\right)-V(x, \tilde{v})\right],
$$


which is nondecreasing in $x \geq \bar{x}^{\star}$. We conclude that $\bar{x}^{\star}$ is optimal on the semi-interval $x \geq \bar{x}^{\star}$.

The foregoing two steps establish that the optimal decision $x^{\star}$ lies in the interval $\left[\underline{x}^{\star}, \bar{x}^{\star}\right]$. For any $x \in\left[\underline{x}^{\star}, \bar{x}^{\star}\right]$, we observe that $(\mathrm{a}) \max _{y \geq x} \mathbb{E}_{\overline{\mathbb{P}}}[V(y, \tilde{v})-V(x, \tilde{v})]=\mathbb{E}_{\overline{\mathbb{P}}}\left[V\left(\bar{x}^{\star}, \tilde{v}\right)-V(x, \tilde{v})\right] \triangleq \bar{g}(x)$ by selecting $y=\bar{x}^{\star}$ and $(\mathrm{b}) \max _{y \leq x} \mathbb{E}_{\mathbb{P}}[V(y, \tilde{v})-V(x, \tilde{v})]=\mathbb{E}_{\mathbb{P}}\left[V\left(\underline{x}^{\star}, \tilde{v}\right)-V(x, \tilde{v}) \triangleq g(x)\right.$ by selecting $y=\underline{x}^{\star}$. By Proposition 1, the optimal solution $x^{\star}$ to the minimax regret problem must satisfy

$$
\bar{g}\left(x^{\star}\right)=\underline{g}\left(x^{\star}\right)=\min _{x \in\left[\underline{x}^{\star}, \bar{x}^{\star}\right]} \max \{\bar{g}(x), \underline{g}(x)\} .
$$

In other words, $\mathbb{E}_{\overline{\mathbb{P}}}\left[V\left(\bar{x}^{\star}, \tilde{v}\right)-V\left(x^{\star}, \tilde{v}\right)\right]=\mathbb{E}_{\mathbb{P}}\left[V\left(\underline{x}^{\star}, \tilde{v}\right)-V\left(x^{\star}, \tilde{v}\right)\right]$. Finally, if we plug existing expressions into this equality then we obtain the optimality condition (12).

Proof of Proposition 3. We first skip the situations where $k^{\star}=0$ or $\bar{v}_{k^{\star}}-\bar{v}_{k^{\star}-1} \geq 2 \theta$. From our reformulation (11), it follows that the regret for the decision $\underline{x}^{\star}$ is $\mathbb{E}_{\overline{\mathbb{P}}}\left[V\left(\bar{x}^{\star}, \tilde{v}\right)-V\left(\underline{x}^{\star}, \tilde{v}\right)\right]$, whereas, for the decision $\bar{x}^{\star}$, the regret is $\mathbb{E}_{\mathbb{P}}\left[V\left(\underline{x}^{\star}, \tilde{v}\right)-V\left(\bar{x}^{\star}, \tilde{v}\right)\right]$. Therefore, we can bound the regret by

$$
\min \left\{\mathbb{E}_{\overline{\mathbb{P}}}\left[V\left(\bar{x}^{\star}, \tilde{v}\right)-V\left(\underline{x}^{\star}, \tilde{v}\right)\right], \mathbb{E}_{\underline{\mathbb{P}}}\left[V\left(\underline{x}^{\star}, \tilde{v}\right)-V\left(\bar{x}^{\star}, \tilde{v}\right)\right]\right\} .
$$

Given the monotonicity of $\mathbb{E}_{\overline{\mathbb{P}}}\left[V\left(\bar{x}^{\star}, \tilde{v}\right)-V(x, \tilde{v})\right]$ and that $\underline{x}^{\star} \geq \bar{x}^{\star}-2 \theta$, we can bound the term $\mathbb{E}_{\overline{\mathbb{P}}}\left[V\left(\bar{x}^{\star}, \tilde{v}\right)-V\left(\underline{x}^{\star}, \tilde{v}\right)\right]$ from above by $\mathbb{E}_{\overline{\mathbb{P}}}\left[V\left(\bar{x}^{\star}, \tilde{v}\right)-V\left(\bar{x}^{\star}-2 \theta, \tilde{v}\right)\right]$, which satisfies

$$
\begin{aligned}
\mathbb{E}_{\overline{\mathbb{P}}}\left[V\left(\bar{x}^{\star}, \tilde{v}\right)-V\left(\bar{x}^{\star}-2 \theta, \tilde{v}\right)\right] & =\frac{1}{N} \sum_{i \in[N]}\left\{2 \theta(p-c)-p\left(\bar{x}^{\star}-\bar{v}_{i}\right)^{+}+p\left(\bar{x}^{\star}-2 \theta-\bar{v}_{i}\right)^{+}\right\} \\
& =2 \theta(p-c)+\frac{1}{N} \sum_{i=1}^{k^{\star}}\left\{p\left(\bar{x}^{\star}-2 \theta-\bar{v}_{i}\right)^{+}-p\left(\bar{x}^{\star}-\bar{v}_{i}\right)\right\} \\
& =2 \theta(p-c)-\frac{1}{N} \sum_{i=1}^{\ell} p \cdot 2 \theta-\frac{1}{N} \sum_{i=\ell+1}^{k^{\star}} p\left(\bar{x}^{\star}-\bar{v}_{i}\right) \\
& \leq 2 \theta(p-c)-2 \theta \cdot \frac{p \ell}{N} .
\end{aligned}
$$

Here the second equality follows from $\bar{v}_{1} \leq \bar{v}_{2} \leq \cdots \leq \bar{v}_{N}$ and $\bar{x}^{\star}=\bar{v}_{k^{\star}}$, and the third line follows from our definition of $\ell$. If $k^{\star}=1$, then $\ell=k^{\star}-1=0$ in the expression just displayed; if $\bar{v}_{k^{\star}}-\bar{v}_{k^{\star}-1} \geq 2 \theta$, then we would have $\ell=k^{\star}-1$.

We can similarly bound $\mathbb{E}_{\mathbb{P}}\left[V\left(\underline{x}^{\star}, \tilde{v}\right)-V\left(\bar{x}^{\star}, \tilde{v}\right)\right]$ by $\mathbb{E}_{\mathbb{P}}\left[V\left(\underline{x}^{\star}, \tilde{v}\right)-V\left(\underline{x}^{\star}+2 \theta, \tilde{v}\right)\right]$, which satisfies

$$
\begin{aligned}
\mathbb{E}_{\mathbb{P}}\left[V\left(\underline{x}^{\star}, \tilde{v}\right)-V\left(\underline{x}^{\star}+2 \theta, \tilde{v}\right)\right] & =\frac{1}{N} \sum_{i \in[N]}\left\{-2 \theta(p-c)+p\left(\underline{x}^{\star}+2 \theta-\underline{v}_{i}\right)^{+}-p\left(\underline{x}^{\star}-\underline{v}_{i}\right)^{+}\right\} \\
& =-2 \theta(p-c)+\frac{1}{N} \sum_{i=1}^{k^{\star}} p \cdot 2 \theta+\frac{1}{N} \sum_{i=k^{\star}+1}^{N} p\left(\underline{x}^{\star}+2 \theta-\underline{v}_{i}\right)^{+} \\
& =-2 \theta(p-c)+\frac{1}{N} \sum_{i=1}^{k^{\star}} p \cdot 2 \theta+\frac{1}{N} \sum_{i=k^{\star}+1}^{j} p\left(\underline{x}^{\star}+2 \theta-\underline{v}_{i}\right) \\
& \leq-2 \theta(p-c)+2 \theta \cdot \frac{p j}{N} .
\end{aligned}
$$


In this expression, the second line is due to $\underline{v}_{1} \leq \underline{v}_{2} \leq \cdots \leq \underline{v}_{N}$ and $\underline{x}^{\star}=\underline{v}_{k^{\star}}$, while the third and fourth lines each follow from the definition of $j$. In both situations - that is, whether $k^{\star}=N$ or $\underline{v}_{k^{\star}+1}-\underline{v}_{k^{\star}} \geq 2 \theta$-we would have $j=k^{\star}$. Hence regret can be bounded from above by (13).

Proof of Proposition 4. We omit the trivial scenario $u=0$ that always leads to a zero regret, which should never be the worst case. Note that, for any $(u, v) \in \mathcal{U}_{1} \times \mathcal{V}_{1}$, the optimal decision of the inner maximization problem over $y$ is $y^{\star}=v / u$. Hence, the model can be simplified to read

$$
\min _{x} \max _{(u, v) \in \mathcal{U}_{1} \times \mathcal{V}_{1}}\left\{(p-c) v-(p-c) u x+p(u x-v)^{+}\right\} .
$$

For any fixed $u \in \mathcal{U}_{1}$, the function $(p-c) v-(p-c) u x+p(u x-v)^{+}$is convex in $v$ and thus achieves its maximum at either $v=\underline{v}_{1}$ or $v=\bar{v}_{1}$. Hence the minimax regret model becomes

$$
\min _{x} \max _{u \in \mathcal{U}_{1}} \max \left\{(p-c) \underline{v}_{1}-(p-c) u x+p\left(u x-\underline{v}_{1}\right)^{+},(p-c) \bar{v}_{1}-(p-c) u x+p\left(u x-\bar{v}_{1}\right)^{+}\right\} .
$$

In addition, for both $v=\underline{v}_{1}$ and $v=\bar{v}_{1}$, the function $(p-c) v-(p-c) u x+p(u x-v)^{+}$is convex in $u$. As a result, we can finally rewrite the minimax regret model as $\min _{x} \max _{i=1,2,3,4}\left\{f_{i}(x)\right\}$ with functions $f_{1}(x)=(p-c) \underline{v}_{1}+p\left(\underline{u}_{1} x-\underline{v}_{1}\right)^{+}-(p-c) \underline{u}_{1} x, f_{2}(x)=(p-c) \bar{v}_{1}+p\left(\underline{u}_{1} x-\bar{v}_{1}\right)^{+}-(p-c) \underline{u}_{1} x$, $f_{3}(x)=(p-c) \underline{v}_{1}+p\left(\bar{u}_{1} x-\underline{v}_{1}\right)^{+}-(p-c) \bar{u}_{1} x$, and $f_{4}(x)=(p-c) \bar{v}_{1}+p\left(\bar{u}_{1} x-\bar{v}_{1}\right)^{+}-(p-c) \bar{u}_{1} x$. Indeed, for any $x$, it must hold that $\min _{x} \max _{i=1,2,3,4}\left\{f_{i}(x)\right\}=\min _{x} \max \left\{f_{2}(x), f_{3}(x)\right\}$. Hence it follows from the convexity of functions $f_{2}$ and $f_{3}$ that the optimal minimax regret order quantity $x_{\mathrm{R}}^{\star}$ lies in the interval $\left[\underline{v}_{1} / \bar{u}_{1}, \bar{v}_{1} / \underline{u}_{1}\right]$ and satisfies the optimality condition $f_{2}\left(x_{\mathrm{R}}^{\star}\right)=f_{3}\left(x_{\mathrm{R}}^{\star}\right)$. Thus we have derived the desired closed-form expression in the theorem.

Proof of Proposition 5. By the definition of our type- $\infty$ Wasserstein ambiguity set, the best-case expected profit $\max _{\mathbb{P} \in \mathcal{F}_{\infty}(\theta)} \mathbb{E}_{\mathbb{P}}[\Pi(x, \tilde{u}, \tilde{v})]$ of an order quantity $x$ is equivalent to

$$
\frac{1}{N} \sum_{i \in[N]} \max _{u \in \mathcal{U}_{i}} \max _{v \in \mathcal{V}_{i}}\left\{(p-c) u x-p(u x-v)^{+}\right\},
$$

which, by noting that $(p-c) u x-p(u x-v)^{+}$is increasing in $v$ for fixed $x$ and $u$, becomes

$$
\frac{1}{N} \sum_{i \in[N]} \max _{u \in \mathcal{U}_{i}}\left\{(p-c) u x-p\left(u x-\bar{v}_{i}\right)^{+}\right\} .
$$

We now observe that, for each $i \in[N], \max _{u \in \mathcal{U}_{i}}\left\{(p-c) u x-p\left(u x-\bar{v}_{i}\right)^{+}\right\}=\min \left\{(p-c) \bar{u}_{i} x,(p-\right.$ $\left.c) \bar{v}_{i}, p \bar{v}_{i}-c \underline{u}_{i} x\right\}$, leading to a best-case expected profit of

$$
\frac{1}{N} \sum_{i \in[N]} \min \left\{(p-c) \bar{u}_{i} x,(p-c) \bar{v}_{i}, p \bar{v}_{i}-c \underline{u}_{i} x\right\} .
$$

Similarly, the worst-case expected profit $\min _{\mathbb{P} \in \mathcal{F}_{\infty}(\theta)} \mathbb{E}_{\mathbb{P}}[\Pi(x, \tilde{u}, \tilde{v})]$ can be re-expressed as

$$
\frac{1}{N} \sum_{i \in[N]} \min _{u \in \mathcal{U}_{i}}\left\{(p-c) u x-p\left(u x-\underline{v}_{i}\right)^{+}\right\} .
$$


For each $i \in[N]$ and fixed $x$, the function $(p-c) u x-p\left(u x-\underline{v}_{i}\right)^{+}$is concave in $u$ and attains its minimum at the boundary point of the interval $\mathcal{U}_{i}=\left[\underline{u}_{i}, \bar{u}_{i}\right]$. Hence,

$$
\begin{aligned}
\min _{u \in \mathcal{U}_{i}}\left\{(p-c) u x-p\left(u x-\underline{v}_{i}\right)^{+}\right\} & =\min \left\{(p-c) \underline{u}_{i} x-p\left(\underline{u}_{i} x-\underline{v}_{i}\right)^{+},(p-c) \bar{u}_{i} x-p\left(\bar{u}_{i} x-\underline{v}_{i}\right)^{+}\right\} \\
& =\min \left\{(p-c) \underline{u}_{i} x, p \underline{v}_{i}-c \bar{u}_{i} x\right\},
\end{aligned}
$$

which implies a worst-case expected profit of $\frac{1}{N} \sum_{i \in[N]} \min \left\{(p-c) \underline{u}_{i} x, p \underline{v}_{i}-c \bar{u}_{i} x\right\}$. Adding up the best- and the worst-case expected profits with weight $\lambda$ yields reformulation (15), which involves a number $2 N$ of concave piecewise affine functions in the objective. Introducing auxiliary epigraphical decision variables $\phi_{i}$ and $\psi_{i}$, we obtain reformulation (16).

Proof of Proposition 6. Note that the sequence $z_{1} \leq z_{2} \leq \cdots \leq z_{2 N+|\mathcal{N}|}$ produces $2 N+|\mathcal{N}|$ intervals on $\mathbb{R}:\left[0, z_{1}\right],\left[z_{1}, z_{2}\right], \ldots,\left[z_{2 N+|\mathcal{N}|-1}, z_{2 N+|\mathcal{N}|}\right]$, and $\left[z_{2 N+|\mathcal{N}|}, \infty\right]$. On the interval $\left[z_{k}, z_{k+1}\right)$ we have

$$
\begin{aligned}
& \sum_{i \in[N]} \min \left\{(p-c) \bar{u}_{i} x,(p-c) \bar{v}_{i}, p \bar{v}_{i}-c \underline{u}_{i} x\right\}=\sum_{i \in[N] \backslash \mathcal{J}_{k}}(p-c) \bar{u}_{i} x+\sum_{i \in \mathcal{J}_{k} \cap\left([N] \backslash \mathcal{L}_{k}\right)}(p-c) \bar{v}_{i}+\sum_{i \in \mathcal{L}_{k}}\left(p \bar{v}_{i}-c \underline{u}_{i} x\right) ; \\
& \sum_{i \in[N]} \min \left\{(p-c) \underline{u}_{i} x, p \underline{v}_{i}-c \bar{u}_{i} x\right\}=\sum_{i \in \mathcal{M}_{k}}\left(p \underline{v}_{i}-c \bar{u}_{i} x\right)+\sum_{i \in[N] \backslash \mathcal{M}_{k}}(p-c) \underline{u}_{i} x .
\end{aligned}
$$

Hence the objective function of the Hurwicz criterion newsvendor model (15) amounts to

$$
\frac{1}{N}\left(\lambda \sum_{i \in[N] \backslash \mathcal{J}_{k}}(p-c) \bar{u}_{i}+(1-\lambda) \sum_{i \in[N] \backslash \mathcal{M}_{k}}(p-c) \underline{u}_{i}-\lambda \sum_{i \in \mathcal{L}_{k}} c \underline{u}_{i}-(1-\lambda) \sum_{i \in \mathcal{M}_{k}} c \bar{u}_{i}\right) x+C,
$$

where $C$ is some constant independent of $x$. Note that as $k$ increases, the index sets $\mathcal{J}_{k}, \mathcal{L}_{k}$, and $\mathcal{M}_{k}$ become larger while the sets $[N] \backslash \mathcal{J}_{k},[N] \backslash \mathcal{L}_{k}$ and $[N] \backslash \mathcal{M}_{k}$ become smaller. We may therefore conclude our proof based on the observation that, along the real line $x \in \mathbb{R}_{+}$, the concave objective function of the Hurwicz criterion model is first increasing and then decreasing in $x$.

Proof of Proposition \%. We first derive the optimal solution to the Hurwicz criterion model. Denote its concave piecewise affine objective function by $h(x)$, whose break points are $x_{\mathrm{A}}=\bar{v}_{1} / \underline{u}_{1}, x_{\mathrm{B}}=\bar{v}_{1} / \bar{u}_{1}$, and $x_{\mathrm{C}}=p \underline{v}_{1} /\left((p-c) \underline{u}_{1}+c \bar{u}_{1}\right)$ with $x_{\mathrm{A}} \geq x_{\mathrm{C}}$ and $x_{\mathrm{A}} \geq x_{\mathrm{B}}$. The corresponding objective values are

$$
\begin{aligned}
h\left(x_{\mathrm{A}}\right)= & \lambda(p-c) \bar{v}_{1}+(1-\lambda) \min \left\{(p-c) \underline{u}_{1} \cdot \frac{\bar{v}_{1}}{\underline{u}_{1}}, p \underline{v}_{1}-c \bar{u}_{1} \cdot \frac{\bar{v}_{1}}{\underline{u}_{1}}\right\} \\
= & \lambda(p-c) \bar{v}_{1}+(1-\lambda)\left(p \underline{v}_{1}-c \frac{\bar{v}_{1} \bar{u}_{1}}{\underline{u}_{1}}\right) ; \\
h\left(x_{\mathrm{B}}\right)= & \lambda(p-c) \bar{v}_{1}+(1-\lambda) \min \left\{(p-c) \underline{u}_{1} \cdot \frac{\bar{v}_{1}}{\bar{u}_{1}}, p \underline{v}_{1}-c \bar{u}_{1} \cdot \frac{\bar{v}_{1}}{\bar{u}_{1}}\right\} \\
= & \begin{cases}\lambda(p-c) \bar{v}_{1}+(1-\lambda)(p-c) \frac{\bar{v}_{1} \underline{u}_{1}}{\bar{u}_{1}} \quad \text { if } p\left(\underline{v}_{1} \bar{u}_{1}-\bar{v}_{1} \underline{u}_{1}\right)-c\left(\bar{v}_{1} \bar{u}_{1}-\bar{v}_{1} \underline{u}_{1}\right) \geq 0 \\
\lambda(p-c) \bar{v}_{1}+(1-\lambda)\left(p \underline{v}_{1}-c \bar{v}_{1}\right) & \text { if } p\left(\underline{v}_{1} \bar{u}_{1}-\bar{v}_{1} \underline{u}_{1}\right)-c\left(\bar{v}_{1} \bar{u}_{1}-\bar{v}_{1} \underline{u}_{1}\right) \leq 0\end{cases} \\
h\left(x_{\mathrm{C}}\right)= & \lambda \min \left\{(p-c) \bar{u}_{1} x,(p-c) \bar{v}_{1}, p \bar{v}_{1}-c \underline{u}_{1} x_{\mathrm{C}}\right\}+(1-\lambda)(p-c) \underline{u}_{1} \frac{\underline{v}_{1}}{(p-c) \underline{u}_{1}+c \bar{u}_{1}} \\
= & \begin{cases}\lambda(p-c) \bar{v}_{1}+(1-\lambda)(p-c) \frac{p \underline{v}_{1} \underline{u}_{1}}{q} \quad \text { if } p\left(\underline{v}_{1} \bar{u}_{1}-\bar{v}_{1} \underline{u}_{1}\right)-c\left(\bar{v}_{1} \bar{u}_{1}-\bar{v}_{1} \underline{u}_{1}\right) \geq 0, \\
\lambda(p-c) \frac{p \underline{v}_{1} \bar{u}_{1}}{q}+(1-\lambda)(p-c) \frac{p \underline{v}_{1} \underline{u}_{1}}{q} \quad \text { if } p\left(\underline{v}_{1} \bar{u}_{1}-\bar{v}_{1} \underline{u}_{1}\right)-c\left(\bar{v}_{1} \bar{u}_{1}-\bar{v}_{1} \underline{u}_{1}\right) \leq 0 ;\end{cases}
\end{aligned}
$$


here $q$ stands for $(p-c) \underline{u}_{1}+c \bar{u}_{1}$. We compare the objective values of these breakpoints as follows.

1. Suppose that $p\left(\underline{v}_{1} \bar{u}_{1}-\bar{v}_{1} \underline{u}_{1}\right)-c\left(\bar{v}_{1} \bar{u}_{1}-\bar{v}_{1} \underline{u}_{1}\right) \geq 0$, in which case $x_{\mathrm{A}} \geq x_{\mathrm{C}} \geq x_{\mathrm{B}}$. Since

$$
h\left(x_{\mathrm{C}}\right)-h\left(x_{\mathrm{A}}\right)=c \bar{u}_{1} \cdot(1-\lambda) \cdot(\underbrace{\frac{\bar{v}_{1}}{\underline{u}_{1}-\frac{p \underline{v}_{1}}{q}}}_{\geq 0})
$$

is always nonnegative, it is sufficient to compare $h\left(x_{\mathrm{C}}\right)$ and $h\left(x_{\mathrm{B}}\right)$, for which we have

$$
h\left(x_{\mathrm{C}}\right)-h\left(x_{\mathrm{B}}\right)=\frac{(p-c)}{q \bar{u}_{1}} \cdot(1-\lambda) \underline{u}_{1} \cdot(\underbrace{p\left(\underline{v}_{1} \bar{u}_{1}-\bar{v}_{1} \underline{u}_{1}\right)-c\left(\bar{v}_{1} \bar{u}_{1}-\bar{v}_{1} \underline{u}_{1}\right)}_{\geq 0}) .
$$

In this scenario, $h\left(x_{\mathrm{A}}\right) \leq h\left(x_{\mathrm{C}}\right), h\left(x_{\mathrm{B}}\right) \leq h\left(x_{\mathrm{C}}\right)$. It follows that $x_{\mathrm{C}}$ is optimal.

2. Now suppose that $p\left(\underline{v}_{1} \bar{u}_{1}-\bar{v}_{1} \underline{u}_{1}\right)-c\left(\bar{v}_{1} \bar{u}_{1}-\bar{v}_{1} \underline{u}_{1}\right) \leq 0$; then $x_{\mathrm{A}} \geq x_{\mathrm{B}} \geq x_{\mathrm{C}}$. Since

$$
h\left(x_{\mathrm{B}}\right)-h\left(x_{\mathrm{A}}\right)=(1-\lambda) c \bar{v}_{1}\left(\frac{\bar{u}_{1}}{\underline{u}_{1}}-1\right)
$$

is always nonnegative, it is sufficient to compare $h\left(x_{\mathrm{B}}\right)$ and $h\left(x_{\mathrm{C}}\right)$, for which we have

$$
\begin{aligned}
h\left(x_{\mathrm{B}}\right)-h\left(x_{\mathrm{C}}\right) & =\lambda(p-c) \bar{v}_{1}+(1-\lambda)\left(p \underline{v}_{1}-c \bar{v}_{1}\right)-\frac{(p-c) p \underline{v}_{1}}{q}\left(\lambda \bar{u}_{1}+(1-\lambda) \underline{u}_{1}\right) \\
& =\frac{\lambda(p-c)\left[\left(c \bar{v}_{1}-p \underline{v}_{1}\right) \bar{u}_{1}+(p-c) \bar{v}_{1} \underline{u}_{1}\right]-(1-\lambda) c\left[\left(c \bar{v}_{1}-p \underline{v}_{1}\right) \bar{u}_{1}+(p-c) \bar{v}_{1} \underline{u}_{1}\right]}{q} \\
& =\frac{(\lambda p-c)}{q}[\underbrace{c\left(\bar{v}_{1} \underline{u}_{1}-c \bar{v}_{1} \bar{u}_{1}\right)-p\left(\bar{v}_{1} \underline{u}_{1}-\underline{v}_{1} \bar{u}_{1}\right)}_{\geq 0}]
\end{aligned}
$$

Here, if $\lambda p \geq c$ then $h\left(x_{\mathrm{B}}\right) \geq h\left(x_{\mathrm{A}}\right)$ and $h\left(x_{\mathrm{B}}\right) \geq h\left(x_{\mathrm{C}}\right)$, which should confirm that $x_{\mathrm{B}}$ is optimal. Otherwise, $x_{\mathrm{C}}$ is optimal.

\section{Appendix B. Newsvendor Model under Discrete Distribution}

It is sufficient to focus on the empirical distribution because any discrete distribution can be transformed to one that is uniformly supported on (not necessarily nonidentical) points. The type- $\infty$ Wasserstein ball of radius zero shrinks to the empirical distribution; that is, $\mathcal{F}_{\infty}(0)=$ $\left\{\frac{1}{N} \sum_{i \in[N]} \delta_{\left(\hat{u}_{i}, \hat{v}_{i}\right)}\right\}$. So whenever $\theta=0$, the Hurwicz criterion model (14) with any $\lambda \in[0,1]$ degenerates into the empirical model:

$$
\max _{x \geq 0}\left\{\lambda \cdot \max _{\mathbb{P} \in \mathcal{F}_{\infty}(0)} \mathbb{E}_{\mathbb{P}}[\Pi(x, \tilde{u}, \tilde{v})]+(1-\lambda) \cdot \min _{\mathbb{P} \in \mathcal{F}_{\infty}(0)} \mathbb{E}_{\mathbb{P}}[\Pi(x, \tilde{u}, \tilde{v})]\right\}=\max _{x \geq 0} \mathbb{E}_{\hat{\mathbb{P}}}[\Pi(x, \tilde{u}, \tilde{v})],
$$

where the random yield and demand jointly follow the discrete empirical distribution.

Since $\bar{u}_{i}=\underline{u}_{i}=\hat{u}_{i}$ and $\underline{v}_{i}=\hat{v}_{i}$ for all $i \in[N]$, a variant of problem (16) gives the following linear program reformulation of the empirical model:

$$
\begin{array}{rll}
\max _{x, \phi} & \frac{1}{N} \sum_{i \in[N]} \phi_{i} & \\
\text { s.t. } & \phi_{i} \leq(p-c) \hat{u}_{i} x \quad \forall i \in[N] \\
& \phi_{i} \leq p \hat{v}_{i}-c \hat{u}_{i} x \quad \forall i \in[N] \\
& x \in \mathbb{R}_{+}, \phi \in \mathbb{R}^{N}, &
\end{array}
$$


for which the list (17) of break points (i.e., extreme points) becomes $\hat{v}_{1} / \hat{u}_{1} \leq \hat{v}_{2} / \hat{u}_{2} \leq \cdots \leq \hat{v}_{|\mathcal{N}|} / \hat{u}_{|\mathcal{N}|}$. Here $\mathcal{N}=\left\{i \in[N]: \hat{u}_{i}>0\right\}$ and we assume, without loss of generality, an increasing order. The index sets in Proposition 6 coincide; that is, $\mathcal{J}_{k}=\mathcal{L}_{k}=\mathcal{M}_{k}=\left\{i \in[N]: \hat{v}_{i} / \hat{u}_{i} \leq \hat{v}_{k} / \hat{u}_{k}\right\}$ for any $k \in \mathcal{N}$. Correspondingly, the characterization (18) in Proposition 6 becomes

$$
\sum_{i \in[N] \backslash \mathcal{J}_{k^{\star}}}(p-c) \hat{u}_{i} \leq \sum_{i \in \mathcal{J}_{k^{\star}}} c \hat{u}_{i} \Longleftrightarrow \sum_{i \in[N] \backslash \mathcal{J}_{k^{\star}}} p \hat{u}_{i} \leq \sum_{i \in[N]} c \hat{u}_{i}
$$

which recovers the known characterization (2) of the optimal order quantity in the model with both demand and yield randomness, given that they follow a discrete joint distribution. We would like to note that the characterization (2) unifies the celebrated characterization of the optimal decision in the newsvendor model with only demand randomness (in which case $\mathcal{N}=[N]$ and $\hat{u}_{i}=1$ for all $i \in \mathcal{N}$ ) or with only yield randomness (for this case the values of $\hat{v}_{i}, i \in \mathcal{N}$, are all equal), provided that the random parameter follows a known discrete distribution.

\section{Appendix C. Newsvendor Model with FGM Copula}

Consider the stochastic newsvendor model (1), the expected profit of an order quantity $x$ under a continuous true demand and yield distribution $\mathbb{P}_{\mathrm{o}}$ with a joint probability density function $f(u, v)$ supported on $[\underline{u}, \bar{v}] \times[\underline{v}, \bar{v}]$ is given as

$$
\mathbb{E}_{\mathbb{P}_{\mathrm{o}}}[\Pi(x, \tilde{u}, \tilde{v})]=(p-c) \cdot \mathbb{E}_{\mathbb{P}_{\mathrm{o}}}[\tilde{u}] \cdot x-p \cdot \int_{\underline{u}}^{\bar{u}} \int_{\underline{v}}^{\bar{v}}(u x-v)^{+} f(u, v) \mathrm{d} v \mathrm{~d} u \triangleq(p-c) \cdot \mathbb{E}_{\mathbb{P}_{\mathrm{o}}}[\tilde{u}] \cdot x-p \cdot \varphi(x) .
$$

Since for fixed values of $u$ and $v$ the profit function $\Pi(x, u, v)$ is concave in $x$, it is not difficult to conclude that problem (1) is a convex problem, for which the optimal order quantity $x_{\mathrm{o}}^{\star}$ can be derived from the first order condition of the preceding expression; that is to say:

$$
\varphi^{\prime}\left(x_{\mathrm{o}}^{\star}\right)=\frac{p-c}{p} \cdot \mathbb{E}_{\mathbb{P}_{\mathrm{o}}}[\tilde{u}] .
$$

Note that we have

$$
\varphi(x)= \begin{cases}\underline{\varphi}(x)=\int_{\underline{u}}^{\bar{u}} \int_{\underline{v}}^{u x}(u x-v) f(u, v) \mathrm{d} v \mathrm{~d} u & \text { if } \bar{u} x \leq \bar{v}, \\ \bar{\varphi}(x)=\int_{\underline{u}}^{\bar{v} / x} \int_{\underline{v}}^{u x}(u x-v) f(u, v) \mathrm{d} v \mathrm{~d} u+\int_{\bar{v} / x}^{\bar{u}} \int_{\underline{v}}^{\bar{v}}(u x-v) f(u, v) \mathrm{d} v \mathrm{~d} u & \text { if } \bar{u} x \geq \bar{v} .\end{cases}
$$

If the true demand and yield distribution $\mathbb{P}_{\mathrm{o}}$ has uniform marginals and is characterized through FGM copula, after some algebraic calculations, it then follows that:

$$
\underline{\varphi}(x)=\frac{\underline{\eta}(\bar{u} x-\underline{v})^{3}-\bar{\eta}(\underline{u} x-\underline{v})^{3}}{6 a b x}+\eta \frac{(a x+b)(\bar{u} x-\underline{v})^{4}+(a x-b)(\underline{u} x-\underline{v})^{4}}{12 a^{2} b^{2} x^{2}}-\eta \frac{(\bar{u} x-\underline{v})^{5}-(\underline{u} x-\underline{v})^{5}}{30 a^{2} b^{2} x^{2}}
$$

with $a=\bar{u}-\underline{u}, b=\bar{v}-\underline{v}, \underline{\eta}=1-\eta$, and $\bar{\eta}=1+\eta$; while

$$
\bar{\varphi}(x)=\underline{\varphi}(x)-\frac{(1+\eta)(\bar{u} x-\bar{v})^{3}}{6 a b x}-\eta \frac{(a x-b)(\bar{u} x-\bar{v})^{4}}{12 a^{2} b^{2} x^{2}}+\eta \frac{(\bar{u} x-\bar{v})^{5}}{30 a^{2} b^{2} x^{2}} .
$$

With these analytical expressions, we can find the optimal order quantity $x_{\mathrm{o}}^{\star}$ under the FGM copula. 


\section{Appendix D. Supplementary Figures of Section 6.1 D.1 Profit CDF with High Profit Margin}
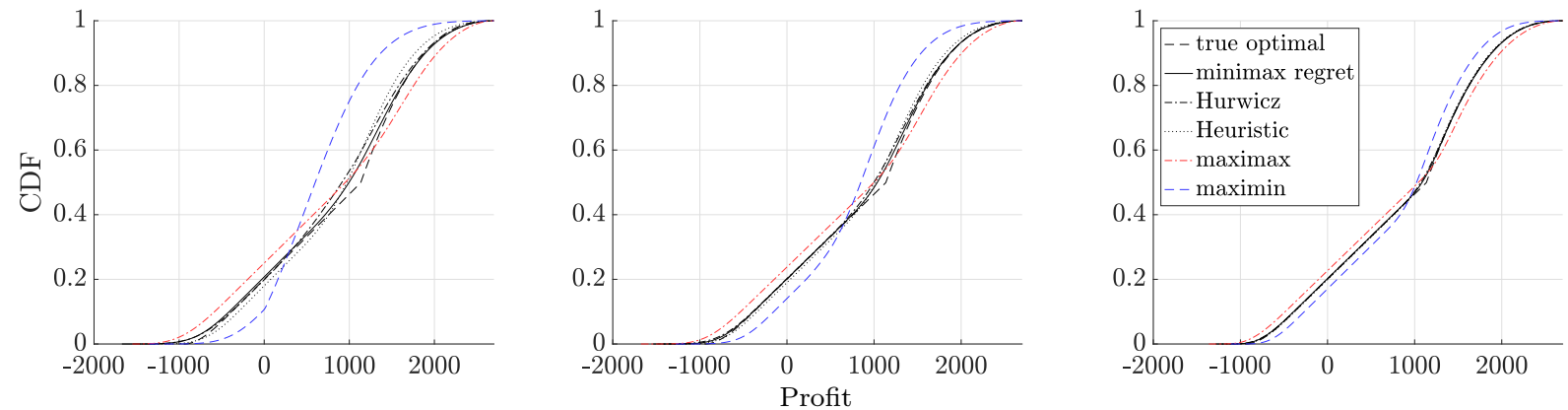

Figure 5 Profit CDF with negative correlated demand and yield of a small sample size $(N=5$, left), a median sample size $(N=15$, middle), and a large sample size $(N=50$, right). Profit margin is high, $c=3$.
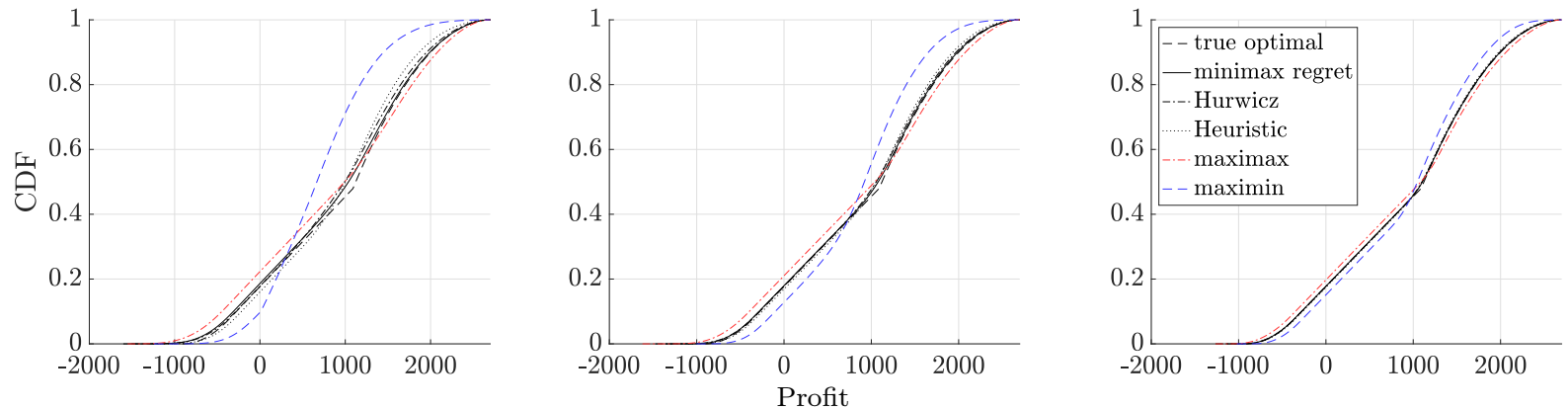

Figure 6 Profit CDF with uncorrelated demand and yield of a small sample size $(N=5$, left $)$, a median sample size $(N=15$, middle), and a large sample size $(N=50$, right $)$. Profit margin is high, $c=3$.
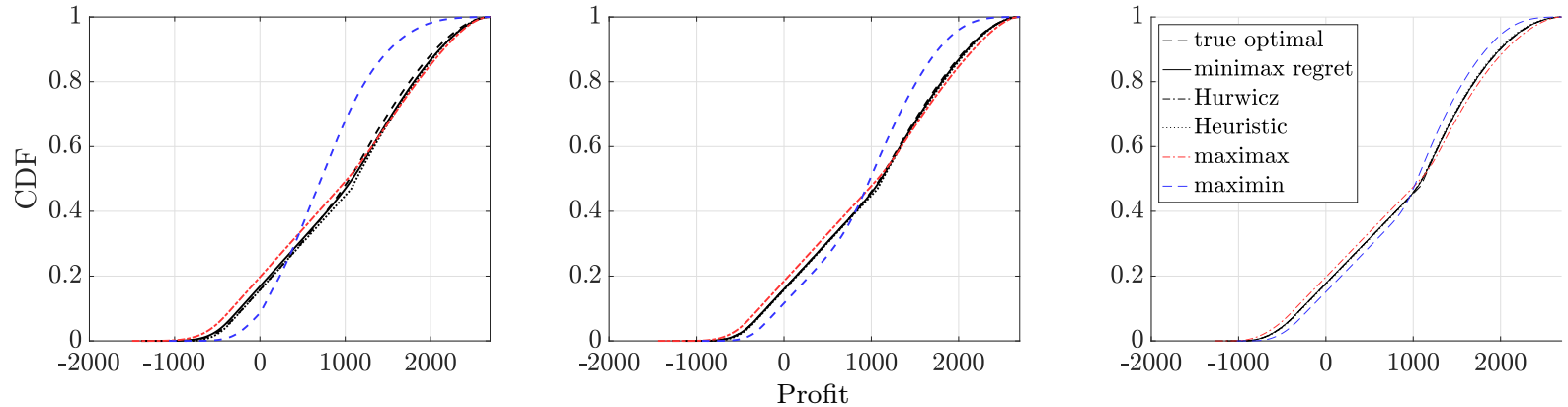

Figure 7 Profit CDF with positive correlated demand and yield of a small sample size $(N=5$, left), a median sample size $(N=15$, middle), and a large sample size $(N=50$, right). Profit margin is high, $c=3$. 


\section{D.2 Profit CDF with Median Profit Margin}
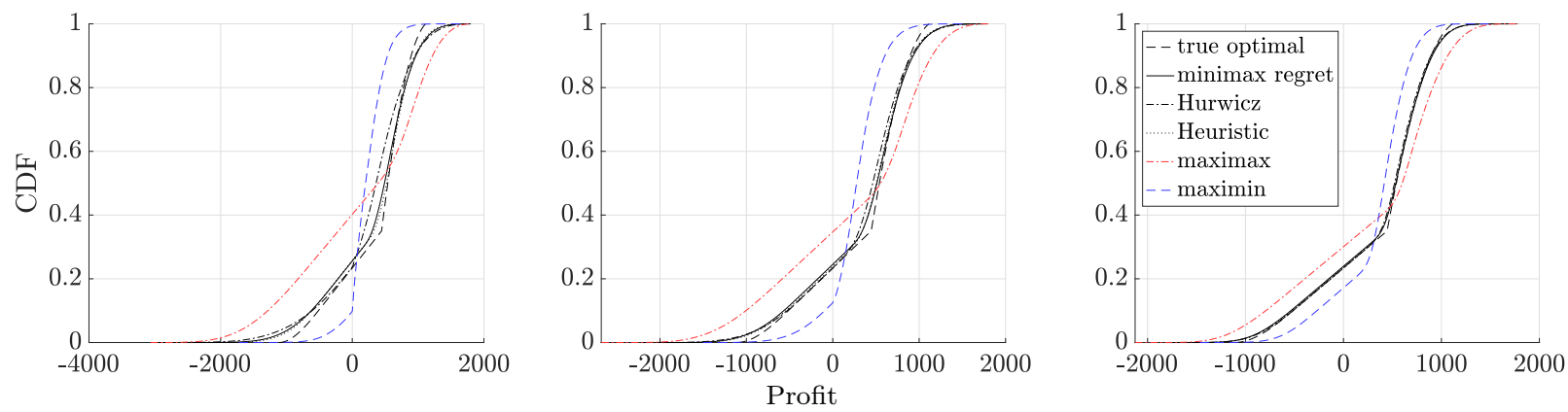

Figure 8 Profit CDF with negative correlated demand and yield of a small sample size $(N=5$, left $)$, a median sample size $(N=15$, middle), and a large sample size $(N=50$, right). Profit margin is median, $c=6$.
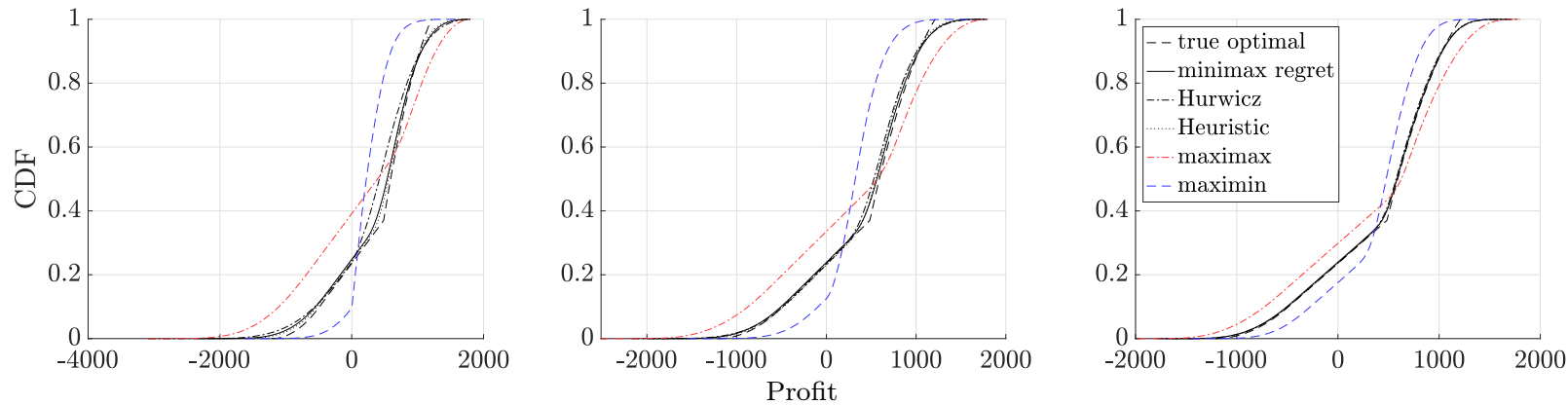

Figure 9 Profit CDF with uncorrelated demand and yield of a small sample size $(N=5$, left $)$, a median sample size $(N=15$, middle), and a large sample size $(N=50$, right $)$. Profit margin is median, $c=6$.
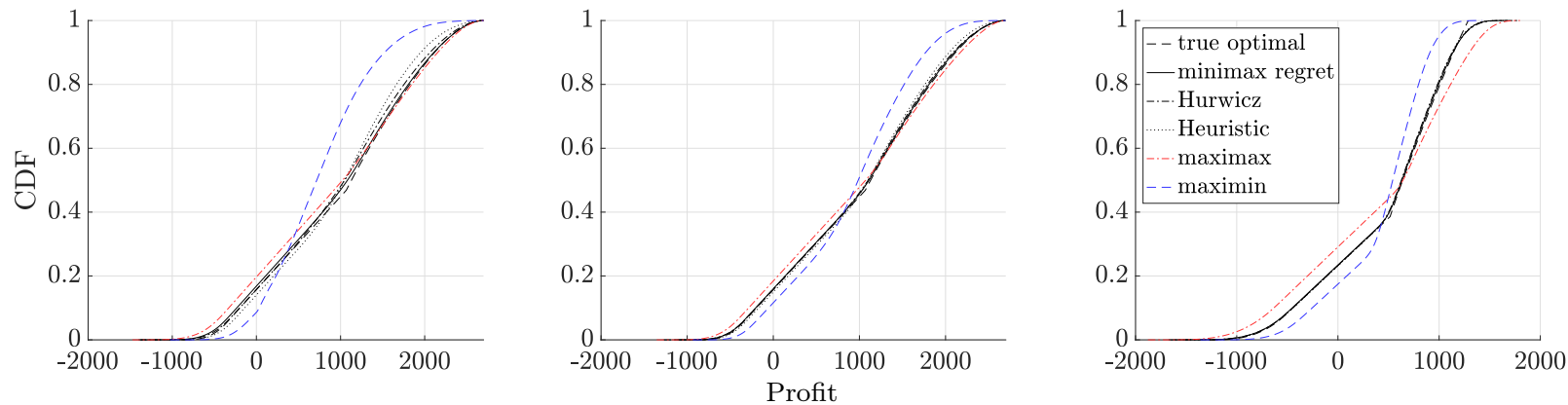

Figure 10 Profit CDF with positive correlated demand and yield of a small sample size $(N=5$, left), a median sample size $(N=15$, middle), and a large sample size $(N=50$, right). Profit margin is median, $c=6$. 


\section{D.3 Profit CDF with Low Profit Margin}
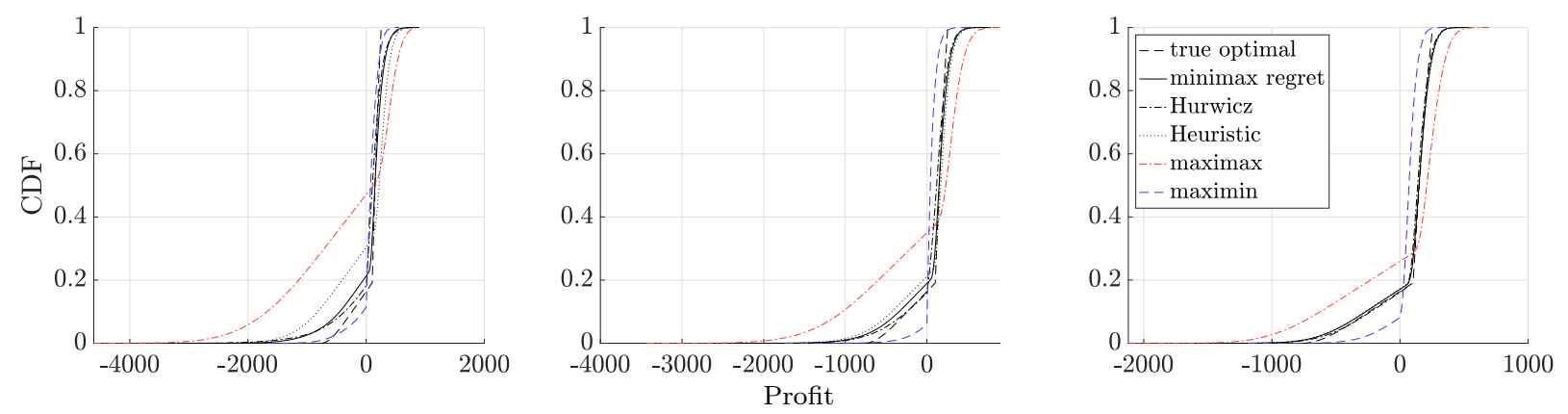

Figure 11 Profit CDF with negative correlated demand and yield of a small sample size $(N=5$, left), a median sample size $(N=15$, middle), and a large sample size $(N=50$, right $)$. Profit margin is low, $c=9$.
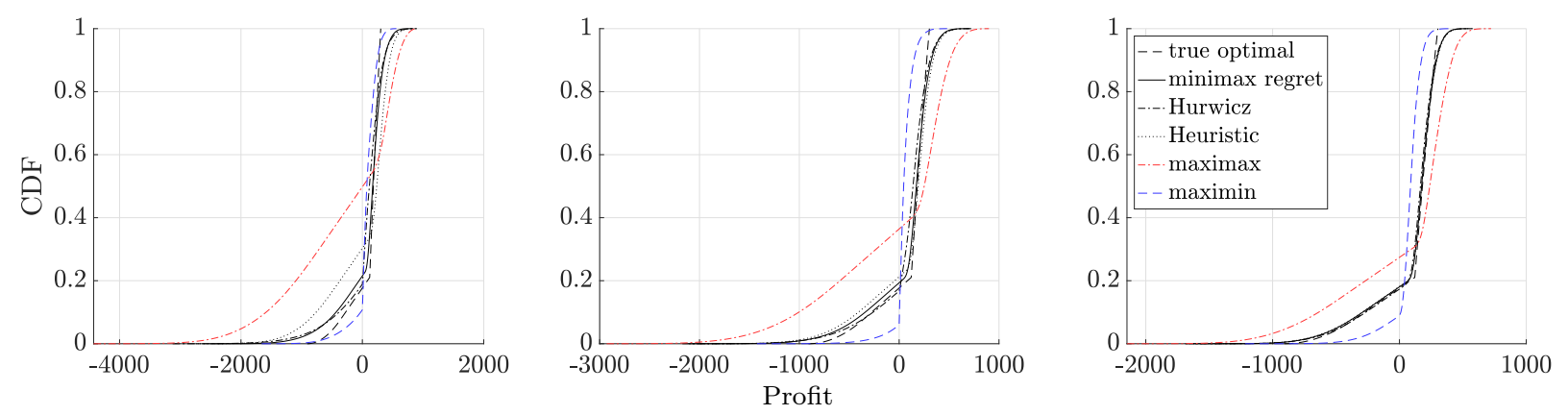

Figure 12 Profit CDF with uncorrelated demand and yield of a small sample size ( $N=5$, left), a median sample size $(N=15$, middle), and a large sample size $(N=50$, right $)$. Profit margin is low, $c=9$.
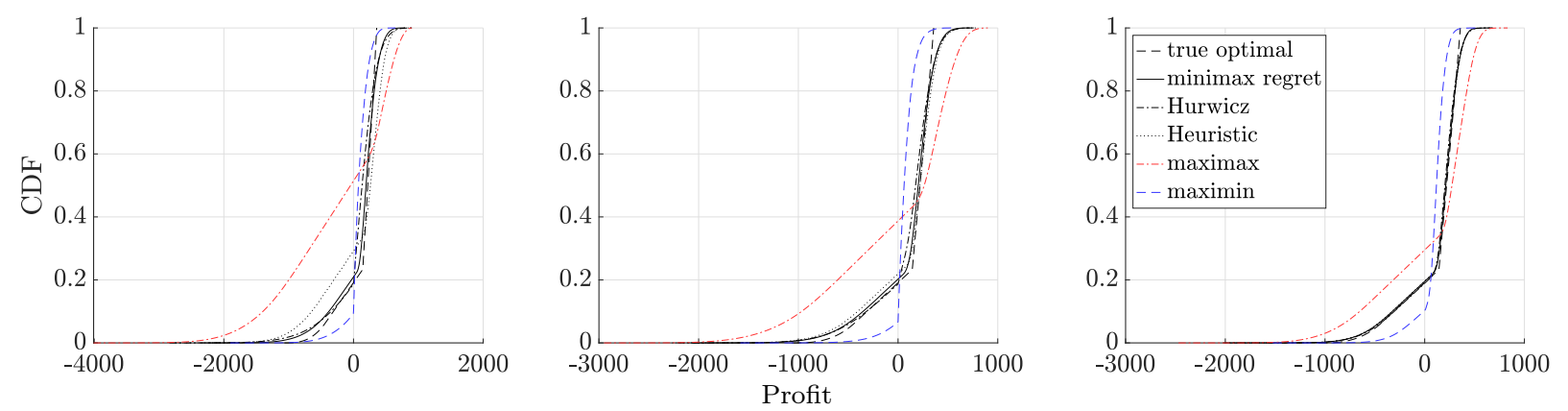

Figure 13 Profit CDF with positive correlated demand and yield of a small sample size $(N=5$, left), a median sample size $(N=15$, middle), and a large sample size $(N=50$, right). Profit margin is low, $c=9$. 


\section{D.4 Average Ratio under Negative Demand and Yield Correlation}
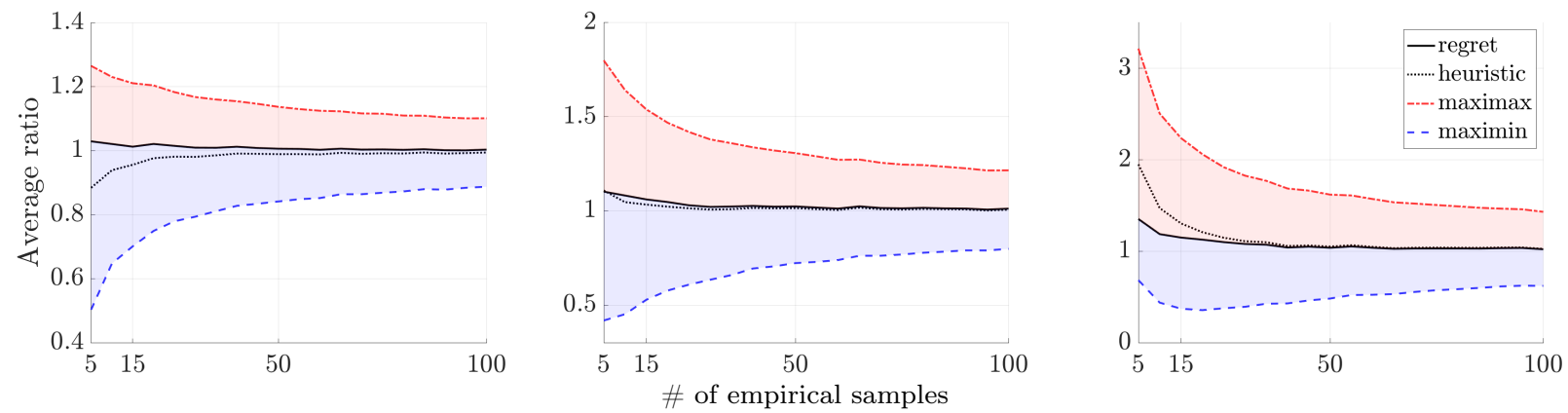

Figure 14 Average ratio to the true optimal order quantity among 1000 random trials. Negative correlated demand and yield under high profit margin $(c=3$, left), median profit $(c=6$, middle), and low profit margin $(c=9$, right).

\section{D.5 Average Ratio under Positive Demand and Yield Correlation}
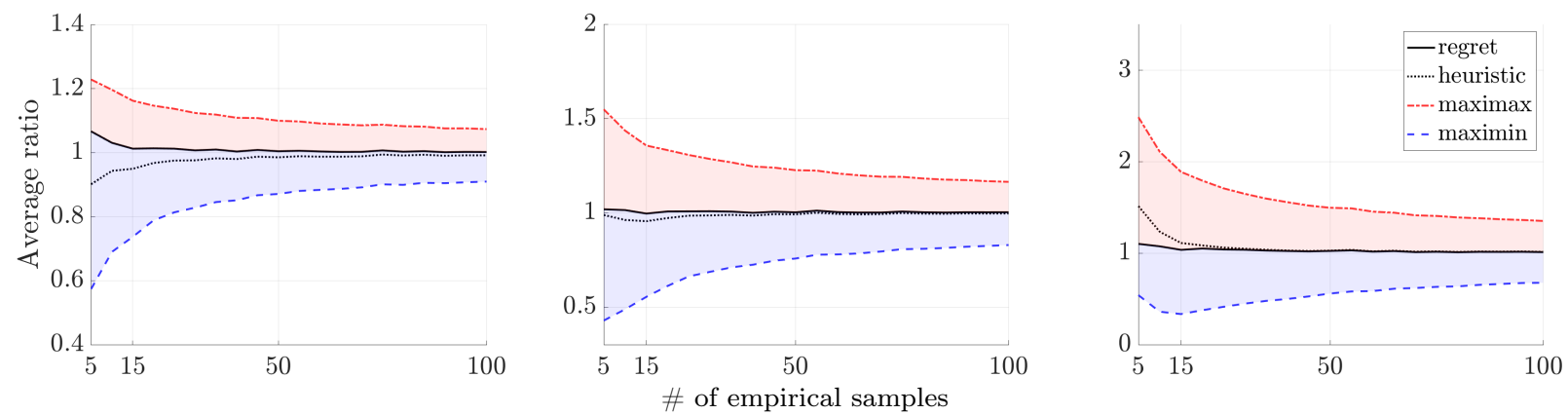

Figure 15 Average ratio to the true optimal order quantity among 1000 random trials. Positive correlated demand and yield under high profit margin $(c=3$, left), median profit $(c=6$, middle), and low profit margin $(c=9$, right). 


\section{Appendix E. Supplementary Figures of Section 6.2 \\ E.1 Profit CDF under Uniform Demand Distribution}
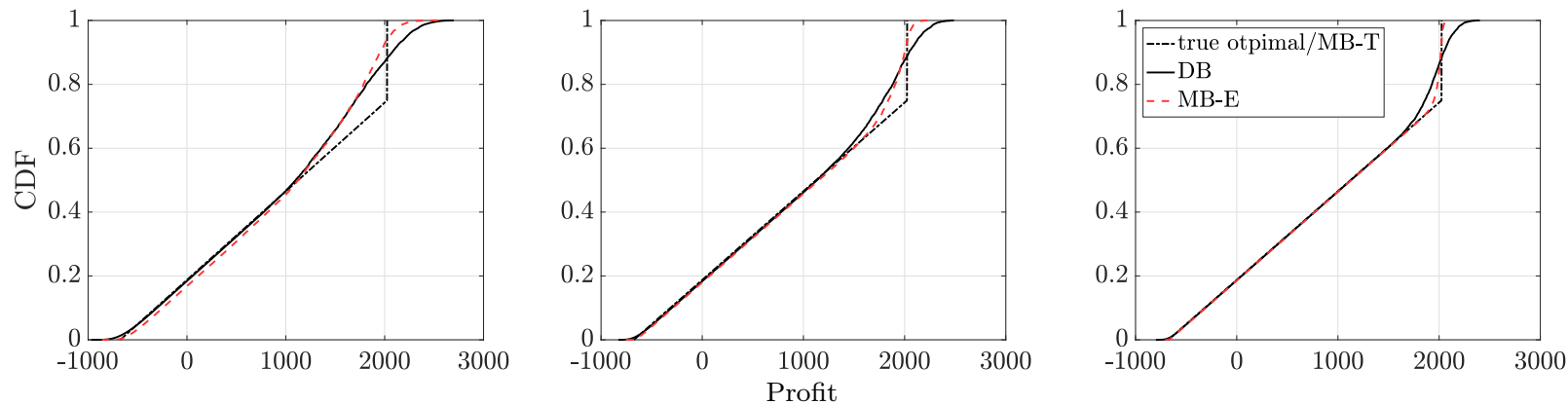

Figure 16 Profit CDF under uniform demand distribution of a small sample size $(N=5$, left $)$, a median sample size $(N=15$, middle), and a large sample size $(N=50$, right). Profit margin is high, $c=3$.
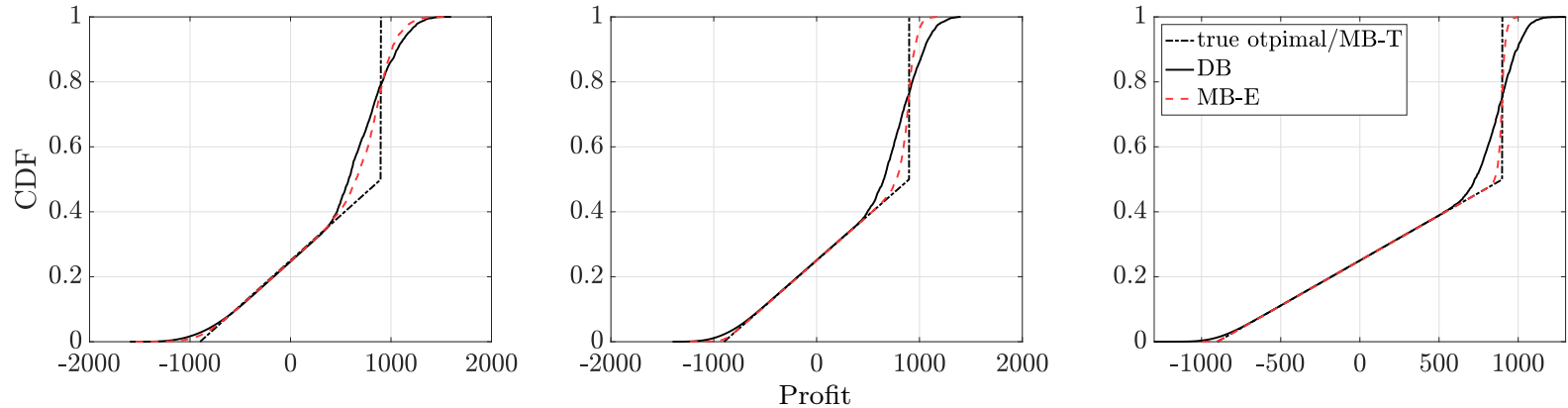

Figure 17 Profit CDF under uniform demand distribution of a small sample size $(N=5$, left $)$, a median sample size $(N=15$, middle), and a large sample size $(N=50$, right). Profit margin is median, $c=6$.
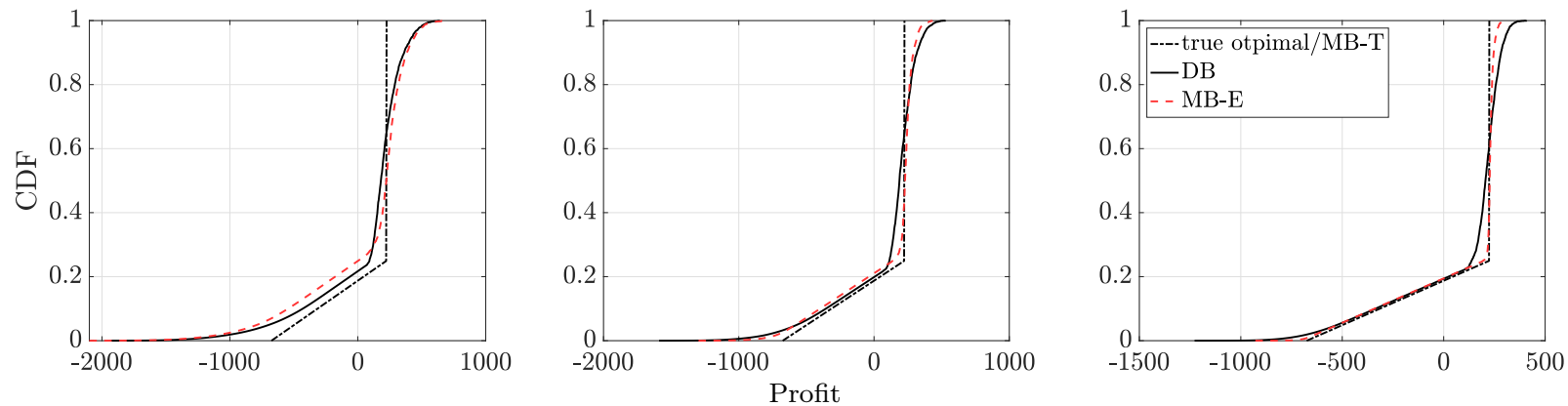

Figure 18 Profit CDF under uniform demand distribution of a small sample size $(N=5$, left $)$, a median sample size $(N=15$, middle), and a large sample size $(N=50$, right). Profit margin is low, $c=9$. 


\section{E.2 Profit CDF under Exponential Demand Distribution}
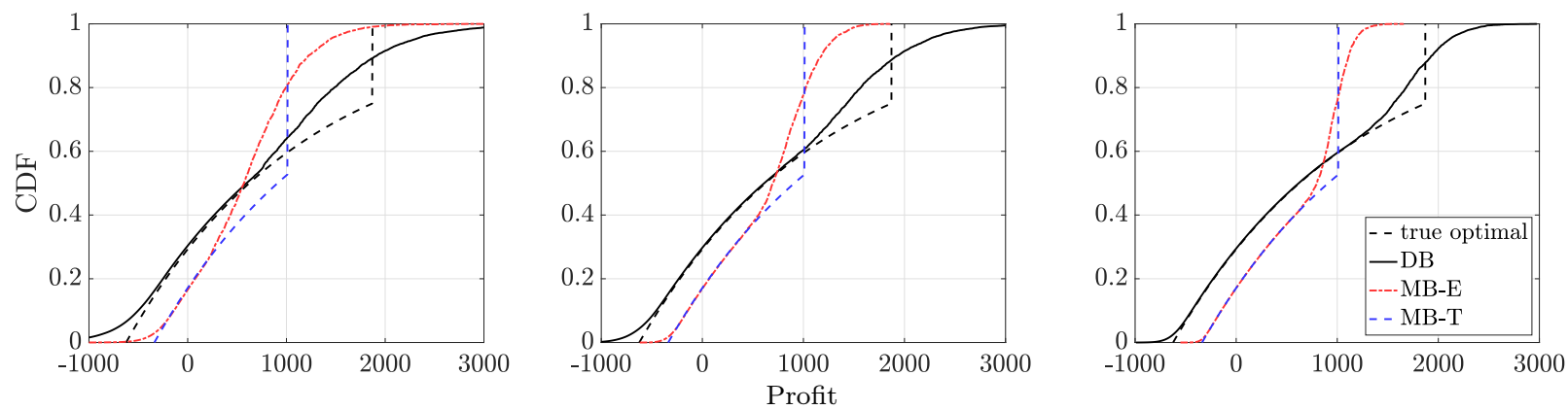

Figure 19 Profit CDF under exponential demand distribution of a small sample size $(N=5$, left $)$, a median sample size $(N=15$, middle), and a large sample size $(N=50$, right). Profit margin is high, $c=3$.
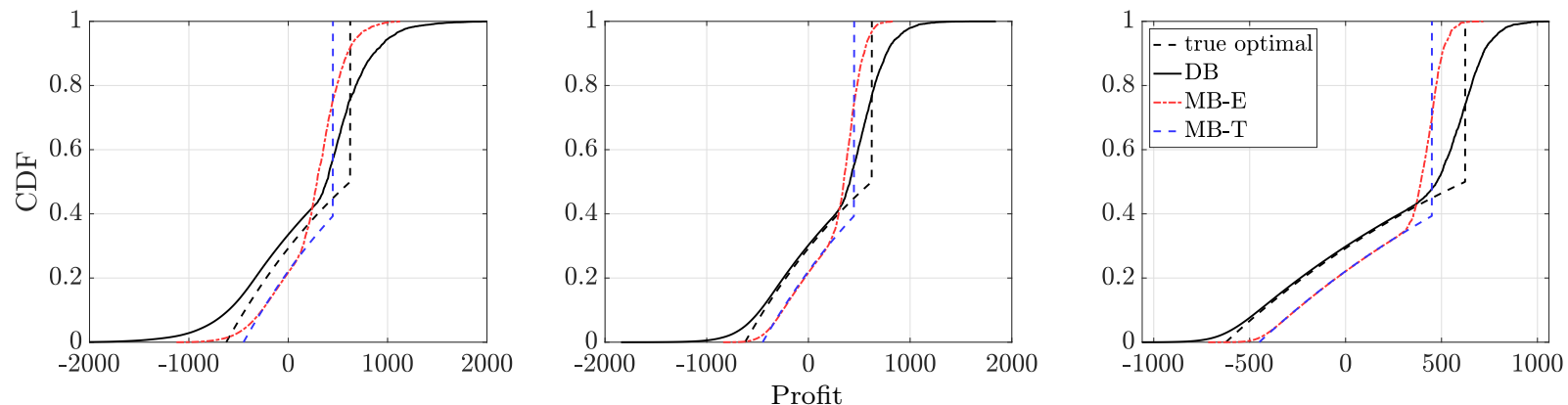

Figure 20 Profit CDF under exponential demand distribution of a small sample size $(N=5$, left $)$, a median sample size $(N=15$, middle), and a large sample size $(N=50$, right). Profit margin is median, $c=6$.
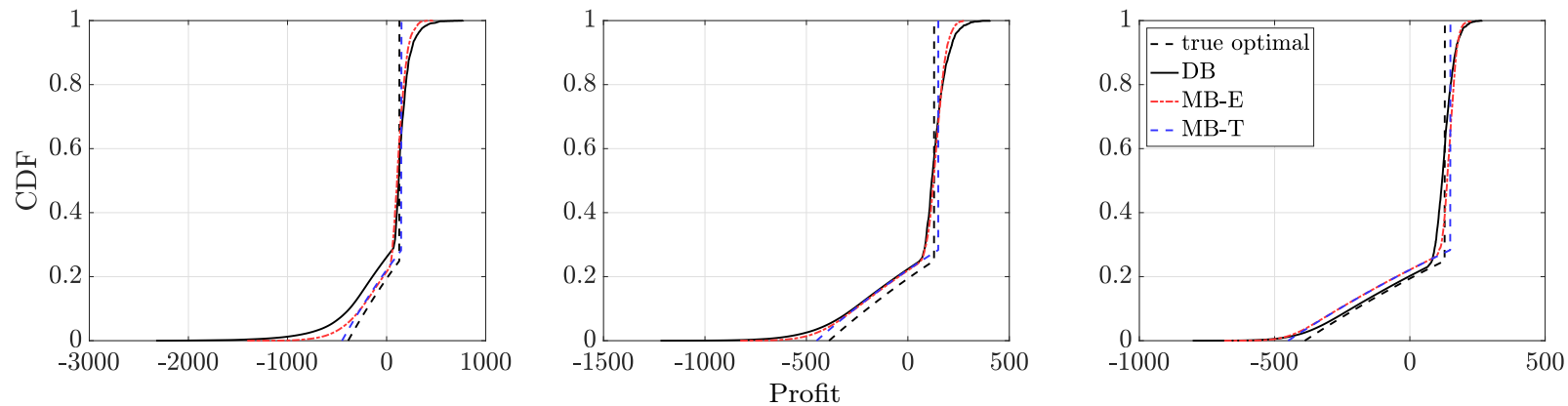

Figure 21 Profit CDF under exponential demand distribution of a small sample size $(N=5$, left $)$, a median sample size $(N=15$, middle), and a large sample size $(N=50$, right). Profit margin is low, $c=9$. 


\section{E.3 Profit CDF under Normal Demand Distribution}
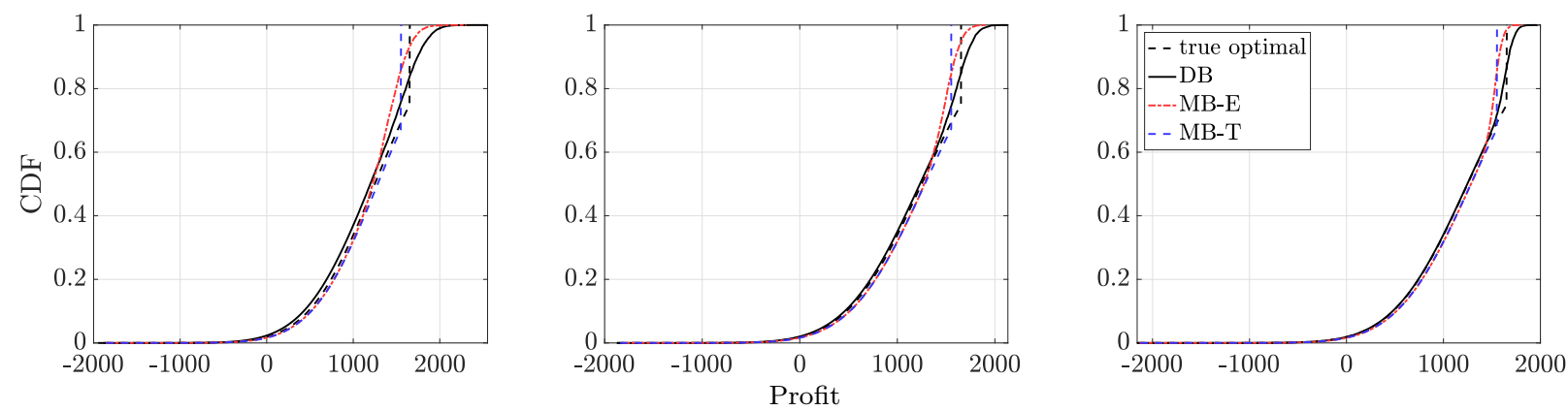

Figure 22 Profit CDF under normal demand distribution of a small sample size $(N=5$, left $)$, a median sample size $(N=15$, middle), and a large sample size $(N=50$, right). Profit margin is high, $c=3$.
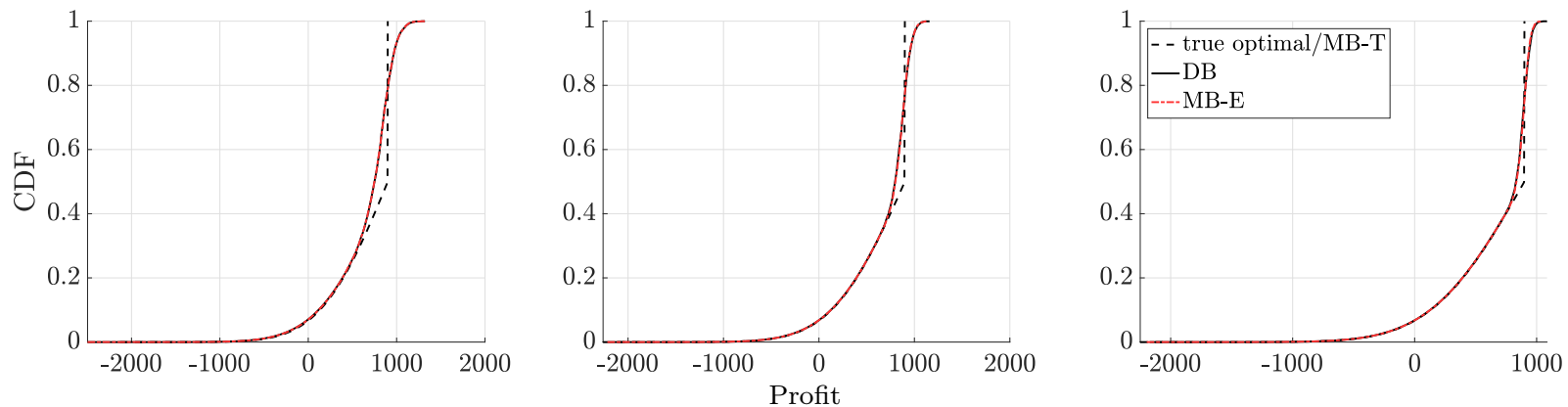

Figure 23 Profit CDF under normal demand distribution of a small sample size $(N=5$, left $)$, a median sample size $(N=15$, middle), and a large sample size $(N=50$, right). Profit margin is median, $c=6$.
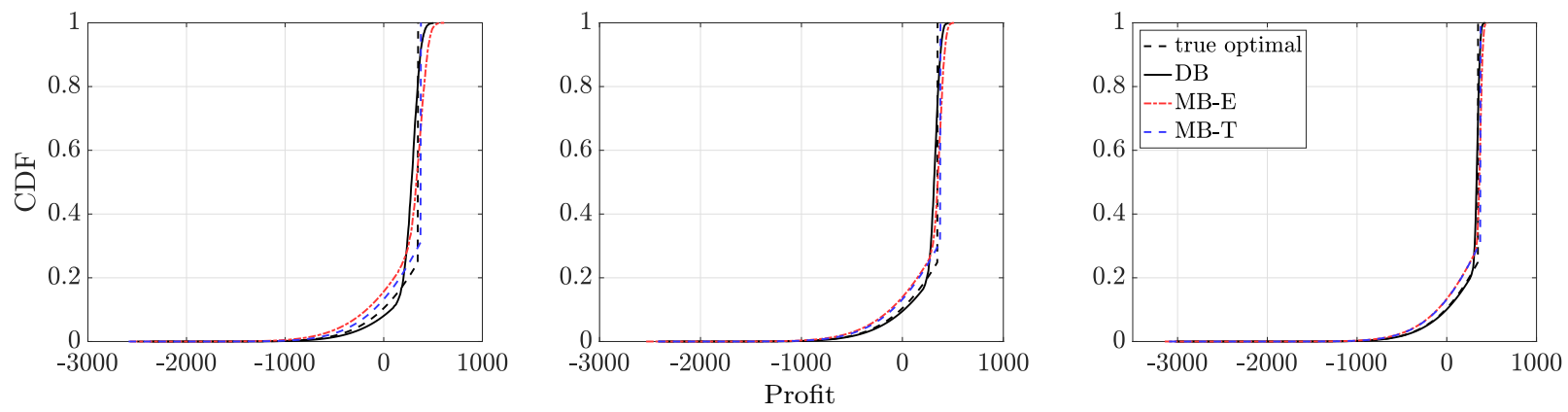

Figure 24 Profit CDF under normal demand distribution of a small sample size $(N=5$, left $)$, a median sample size $(N=15$, middle), and a large sample size $(N=50$, right $)$. Profit margin is low, $c=9$. 